\title{
NANOSCIENCE AND TECHNOLOGY AN INTERNATIONAL JOURNAL
}

\author{
SERGEY A. LURIE \\ Institute of Applied Mechanics of \\ Russian Academy of Science \\ Moscow, Russia
}

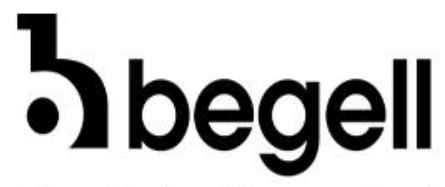

New York • Connecticut 


\section{AIMS AND SCOPE}

Nanoscience and Nanotechnology: An International Journal (NST) is a global forum for the communication of comprehensive articles on nanoscience and nanotechnology research, especially interdisciplinary studies at the interfaces of materials science, physics, chemistry, biology, electronics, energy, and engineering.

Among the areas of interest to the journal are:

- $\quad$ Synthesis and processing of inorganic, organic and hybrid nanosized materials

- Theory, modeling and simulation of nanometer-scale objects and synthetic, assembly, and interaction processes

- $\quad$ Crystal structure, physical properties and characterization of nanomaterials and nanodevices

- Application of novel nanostructures and nanodevices in photonics, electronics, energy harvesting, energy storages, and bio-medical

Nanoscience and Technology: An International Journal (ISSN 2572-4258) is published quarterly and owned by Begell House, Inc., 50 North Street, Danbury, CT 06810, telephone (203) 456-6161. USA subscription rate for 2018 is \$1,155.00. Add $\$ 10.00$ per issue for foreign airmail shipping and handling fees to all orders shipped outside the United States or Canada. Subscriptions are payable in advance. Subscriptions are entered on an annual basis, i.e., January to December. For immediate service and charge card sales, call (203) 456-6161 Monday through Friday 9 AM-5 PM EST. Fax orders to (203) 456-6167. Send written orders to Subscriptions Department, Begell House, Inc., 50 North Street, Danbury, CT 06810. You can also visit our website at http://www.begellhouse.com/ or http://www.dl.begellhouse.com/.

This journal contains information from authentic and highly regarded sources. Reprinted material is quoted with permission, and sources are indicated. A wide variety of references is listed. Reasonable efforts have been made to publish reliable data and information, but the editor and the publisher assume no responsibility for any statements of fact or opinion expressed in the published papers or in the advertisements.

Copyright (c) 2018 by Begell House, Inc. All rights reserved. Printed in the United States of America. Authorization to photocopy items for internal or personal use, or the internal or personal use of specific clients, is granted by Begell House, Inc., for libraries and other users registered with the Copyright Clearance Center (CCC) Transactional Reporting Service, provided that the base fee of $\$ 35.00$ per copy, plus .00 per page, is paid directly to CCC, 27 Congress St., Salem, MA 01970, USA. For those organizations that have been granted a photocopy license by CCC, a separate payment system has been arranged. The fee code for users of the Transactional Reporting Service is: [ISSN 2572-4258 /18/ $\$ 35.00+\$ 0.00$ ]. The fee is subject to change without notice.

Begell House, Inc.'s, consent does not extend to copying for general distribution, for promotion, for creating new works, or for resale. Specific permission must be obtained from Begell House, Inc., for such copying.

Printed October 3, 2018 


\title{
NANOSCIENCE AND TECHNOLOGY AN INTERNATIONAL JOURNAL
}

\author{
EDITOR-IN-CHIEF \\ SERGEY A. LURIE \\ Institute of Applied Mechanics of \\ Russian Academy of Science \\ Moscow, Russia \\ salurie@mail.ru \\ EXECUTIVE SECRETARY \\ YULIA KARNET \\ Institute of Applied Mechanics of \\ Russian Academy of Sciences \\ Moscow, Russia
}

\section{EDITORIAL BOARD MEMBERS}

Elias Aifantis

Laboratory of Mechanics and Materials

School of Engineering, Aristotle University of

Thessaloniki, Thessaloniki, Greece

E-mail: mom@mom.gen.auth.gr

Pulickel AJayan

Benjamin M. and Mary Greenwood Anderson Professor of Engineering, Professor and Founding Chair of

Materials Science and NanoEngineering

Rice University, Houston, TX, USA

Email: ajayan@rice.edu

\section{DEJI AKINWANDE}

Associate Professor of Electrical and Computer

Engineering, The University of Texas at Austin

MER 1.206L, R9900

10100 Burnet Rd. Bldg 160, Austin, TX, USA

Email: deji@ece.utexas.edu

\section{IVAN ARgatov}

Institute of Mathematics and Physics

Aberystwyth University, Finland

Email: ivan.argatov@gmail.com

\section{VICTOR EREMEYEV}

Faculty of Mechanical Engineering and Aeronautics

Rzeszów University of Technology, Poland

Email: eremeyev.victor@gmail.com

\section{YURY GOGOTSI}

Distinguished University and Trustee Chair Professor

Nanomaterials Group, Department of Materials Science and Engineering, Drexel University

Philadelphia, PA, USA

Email: gogotsi@drexel.edu
MiCHELE BRUN

Department of Mechanical, Chemical and

Material Engineering

University of Cagliari, Italy

Email: mbrun@unica.it

LOREDANa CONTRAFatTo

Engineering Department of Civil and

Architectural

Section of Structural Engineering,

University of Catania, Italy

Email: loredana.contrafatto@dica.unict.it

\section{Massimo Cuomo}

University of Catania

Piazza Università,

2, 95124 Catania CT, Italy Email:

mcuomo@dica.unict.it

FRANCESCO DELL'ISOLA

Università degli Studi di Roma La Sapienza

Dipartimento di Ingegneria Strutturale e

Geotecnica, Via Eudossiana

Roma, Italy

Email: francesco.dellisola@uniroma1.it

ANDREAS OECHSNER

Head, Mechanical Engineering

Gold Coast Campus, Australia

Email: a.oechsner@griffith.edu.au

LUCA Placid

International Telematic University

UNINETTUNO

Corso Vittorio Emanuele II, 39, Roma Italy

Email: luca.placidi@uninettunouniversity.net 
ANNA KNYAZEVA

Institute of Computational Technologies

SB RAS, Tomsk Polytechnic University

Tomsk, Russian Federation

Email: agknyazeva@tpu.ru

SANG BoK LEE

Professor of Chemistry and Biochemistry

Deputy Director,

DOE Nanostructures for Electrical, Energy Storage

(NEES) EFRC

University of Maryland, College Park, MD, USA

Email: slee@umd.edu

\section{GaO LIU}

Group Leader, Applied Energy Materials Group

Energy Storage and Distributed Resources Div.

Lawrence Berkeley National Laboratory

Berkeley, CA, USA

Email: Gliu@lbl.gov

GENNADY MISHURIS

Institute of Mathematics and Physics

Aberystwyth University, UK

Email: ggm@aber.ac.uk
IgOR SEVostianov

New Mexico State University

1780 E University Ave, Las Cruces, NM USA

Email: igor@nmsu.edu

YURY SOLYAEV

Institute of Applied Mechanics of

Russian Academy of Sciences, Russia

Email: Solyaevyos@iam.ras.ru

Vladimir STEgaILOV

Moscow Physical and Technical University

Tallinskaya, 34, Moscow, Russia

Email: v.stegailov@hse.ru

Petr Trusov

Perm Polytechnic Institute

(Technical University), Russia

Email: tpv@matmod.pstu.ac.ru

ANDREW WEE

Professor Department of Physics

Vice President, University and Global Relations

National University of Singapore

2 Science Drive 3, Singapore

Email: wee-office@nano.acs.org 


\section{NANOSCIENCE AND TECHNOLOGY AN INTERNATIONAL JOURNAL}

VOLUME 9, IsSUE 4

\section{CONTENTS}

Modeling of Subgrain's Crystallographic Misorientation Distribution

283 N.S. Kondratev \& P.V. Trusov

A Variant of Describing Adhesion Interaction in the Probe-Sample System of an Atomic-Force Microscope

V.Yu. Presnetsova, S.N. Romashin, L.Yu. Frolenkova, V.S. Shorkin, \& S.I. Yakushina

Effects of Viscous Dissipation and Heat Generation/Absorption on Nanofluid Flow over an Unsteady Stretching Surface with Thermal Radiation and Thermophoresis

A.K. Pandey \& M. Kumar

MHD Mixed Convection in Trapezoidal Enclosures Filled with Micropolar Nanofluids

S.E. Ahmed, A.K. Hussein, M.A. Mansour, Z.A. Raizah, \& X. Zhang

Index, Volume 9, 2018 



\title{
MODELING OF SUBGRAIN'S CRYSTALLOGRAPHIC MISORIENTATION DISTRIBUTION
}

\author{
N.S. Kondratev ${ }^{1, *}$ E P.V. Trusov ${ }^{2}$ \\ ${ }^{1}$ Research Institute of Mechanics, Lobachevsky State University, \\ Nizhni Novgorod, 603600, Russia \\ ${ }^{2}$ Perm National Research Polytechnic University, Perm, 614990, Russia \\ *Address all correspondence to: Nikita Sergeevich Kondratev, Research Institute \\ of Mechanics, Lobachevsky State University of Nizhni Novgorod, 23 Gagarin Ave., \\ Bld. 6, Nizhny Novgorod, 603600, Russia, E-mail: kondratevns@gmail.com
}

\begin{abstract}
At present the actual task of various industries is to create products from metal polycrystals and their alloys with the required properties and characteristics. The processing of such products is generally carried out with intense elastoplastic deformation, and is often accompanied by temperature effects. At the same time, the structure of materials undergoes substantial rearrangements, which leads to a significant change in their properties. An effective tool for solving the problem of creating products with the necessary characteristics is the use of multilevel elastoplastic models that allow one to investigate the internal structure of a material in numerical experiments. In the developed two-level statistical model of inelastic deformation, there is the problem of selecting (forming) heterogeneous elements (subgrains) in the initially approximately homogeneous grain. The main source of inhomogeneity is the appearance of crystallographic misorientation of parts (subgrains) of the original grains with respect to one another. The paper considers the problem of determining the orientation of subgrains in the initial stages of inelastic deformation. The mechanism of formation of incidental cell boundaries as a consequence of the appearance of dislocation walls is considered. The method to model boundaries of this type is proposed. In the reference configuration of deformation, flat sections of the cell boundaries (facets) are randomly assigned according to the uniform law. It is supposed that some of mobile dislocations "settle" on the prescribed incidental boundaries. It is shown that due to the appearance of cell boundaries the angle of the subgrain's crystallographic misorientation is proportional to the dislocation shears in the grain. Calculations are made of the uniaxial stretching of a copper polycrystal. Satisfactory agreement between numerical results of the subgrains misorientation distribution with respect to the initial grain and experimental data is shown. The model also makes it possible to take into account the decrease in the number of active slip systems in subgrains during rotation.
\end{abstract}

KEY WORDS: subgrains misorientation, fragmentation, low-angle boundaries, crystal plasticity

\section{INTRODUCTION}

Thermomechanical treatment of metals and alloys provides wide possibilities of forming grain (subgrain) and defective structure of the material, the current state of which determines the properties of materials. Plastic deformation with elevated 
temperatures or subsequent heating of polycrystals followed by cold inelastic deformation are accompanied by a variety of high-temperature processes at various scale levels. They are recovery, coalescence of subgrains, polygonization, recrystallization, and phase transitions (Gorelik et al., 2005). Trusov and Kondratev (2018) consider the problem of modeling static recrystallization. In this work, the recrystallization mechanism, according to which the initially existing sections of high-angle grain boundaries begin to migrate into more defective grains after preliminary plastic deformation is investigated. The recrystallization nuclei in this case are either individual cells or cell blocks (depending on the material) located near the grain boundary. The migration boundaries of recrystallization nucleus appear due to the difference in the volume energy stored in the defects, the surface energy of the grain boundary impedes its migration according to the Bailey-Hirsch criterion (Bailey and Hirsch, 1962). The considered model (Trusov and Kondratev, 2018) is a two-level one. Internal variables of the model are assumed to be homogeneous on the scale of an individual grain, as well as the stress-strain state of the mesolevel element (grain level), which largely determines the speed of their computation. In view of this, the problem of introducing cells and/or their blocks into the consideration of the initial stage of plastic deformation and the subsequent description of the evolution of their crystallographic misorientation and geometric shape is one of the main topics in the problem of physicomathematical modeling of static recrystallization. The problem of modeling the form is beyond the scope of the study of the present paper. Misorientation and geometric shape are entered into expression for the Bailey-Hirsch recrystallization criterion, and therefore determine the kinetics of this process. Experimental data indicate that even with small degrees of plastic deformation due to its inhomogeneity, there occur the processes of rotation of the grain's parts relative to one another (fragmentation process) (Koneva, 1998; Koneva and Kozlov, 2004; Hansen, 1990; KuhlmannWilsdorf, 1989; Kuhlmann-Wilsdorf and Hansen, 1991; Kuhlmann-Wilsdorf and Thompson, 1977). The evolution of such rotations is determined by a variety of factors: temperature, crystallographic orientation of grains relative to the characteristic directions of deformation, stacking fault energy (SFE), physical mechanisms of inelastic deformation, strain rate, grain size, lattice type, deformation degree, loading complexity, and a number of other factors (Liu and Hansen, 1995; Kuhlmann-Wilsdorf and Hansen, 1991; Gorelik et al., 2005, et al.). At the initial stage of cold quasi-static plastic deformation, the fragmentation process is largely associated with the mechanism of formation of incidental cell boundaries (Liu and Hansen, 1995). This is realized due to the slip of dislocations along the primary systems, their trapping by defects of various nature, and further alignment in dislocation walls. The most significant factor for this mechanism is the crystallographic orientation of the grain lattice with respect to the characteristic loading axes. The aim of the paper is to model the cell's misorientation distribution under inelastic deformation. 


\section{MECHANISM OF FORMATION OF LOW-ANGLE DISLOCATION BOUNDARIES}

Plastic deformation leads to the movement and redistribution of dislocations. Consequently, regions saturated with dislocations (dislocation boundaries) and regions practically free of defects are formed in grains (Gracio et al., 1989; Liu and Hansen, 1995). The appearance of dislocation boundaries leads to further rotations of the parts of the crystal and to the refinement of the grain structure. For many crystalline materials, the sequence of such processes takes place at different scale levels (Koneva, 1998; Koneva and Kozlov, 2004), which have a significant effect on the behavior of metals during plastic deformation and physical and mechanical characteristics of finished products (Gorelik et al., 2005; Vishnyakov et al., 1979).

Many theoretical and experimental papers have been devoted to the investigation of this problem. In particular, in the following works (Hansen, 1990; KuhlmannWilsdorf, 1989; Kuhlmann-Wilsdorf and Hansen, 1991; Kuhlmann-Wilsdorf and Thompson, 1977), based on the analysis of the experimental data, classification and description of the subgrain's structure at different plastic strain intensities are set. In the cited works, the formation of subgrain structures in polycrystalline materials with high and medium SFE subject to cold deformation is analyzed.

Polycrystals with high and medium SFE belong to materials with a low-energy dislocation structure (Kuhlmann-Wilsdorf, 1989; Kuhlmann-Wilsdorf and Hansen, 1991). During the process of plastic deformation, dislocations interact with one another, striving to form clusters. The rearrangement of dislocations into clusters occurs in both polycrystalline materials and single crystals. The boundaries of these clusters have a high density of dislocations, their interior is the regions of crystallites with a relatively low dislocation density. The most frequent dislocation configurations are two-dimensional dislocation boundaries (dislocation walls), dislocation tangles, and three-dimensional dislocation cellular structures. To describe formation of various dislocation configurations, the principle of minimizing internal energy is often used, proposed and developed in the works of KulmanWilsdorf and co-authors (Kuhlmann-Wilsdorf, 1989; Kuhlmann-Wilsdorf and Thompson, 1977, et al.). According to this principle, during the plastic deformation, dislocation configurations gradually approach the structures having a minimum internal energy per unit length of the dislocation line. In these structures, as the deformation proceeds, the density of dislocations increases, hence, the energy of interaction between dislocations increases. Uniform dislocation distribution by grain volume is not a stable configuration. In this case, the energy stored on dislocations can be reduced by forming clusters of dislocations that screen the stress fields of each other. Regularities in the formation of dislocation structures are very complicated (Hansen, 1990; Kuhlmann-Wilsdorf, 1989; Kuhlmann-Wilsdorf and Hansen, 1991; Kuhlmann-Wilsdorf and Thompson, 1977). As a rule, the following property is correct: the greater the active slip systems, the smaller is the specific energy of the dislocation boundary (Hansen, 1990).

Volume 9, Issue 4, 2018 


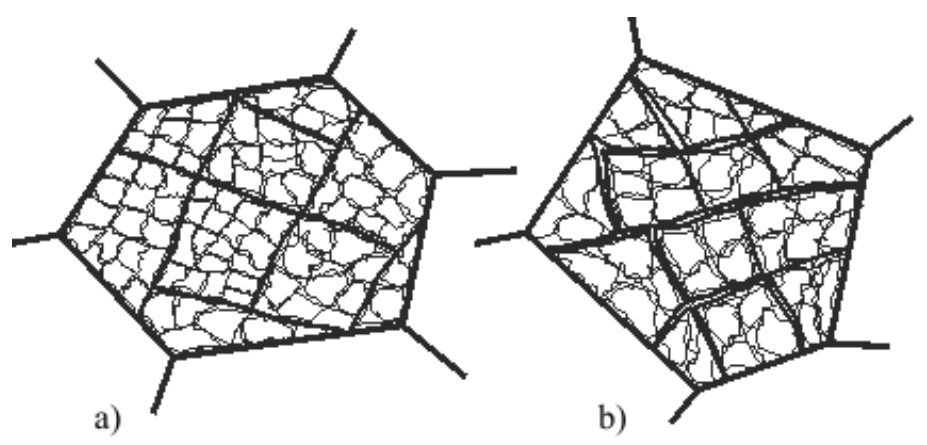

FIG. 1: The scheme of subgrain structure at low (a) and average values (b) of deformation based on Hansen (1990). Dislocation walls in the figure are marked by the thin line, dense dislocation walls are denoted by one thick line, microbands are represented by the double thick line

Misorientation of grain parts during plastic deformation occurs at three different scale levels (Kuhlmann-Wilsdorf and Hansen, 1991). The smallest in scale level disoriented elements in the grain are individual cells. They are separated by incidental dislocation boundaries or dislocation cell boundaries (Fig. 1). It is possible that the cells are the nuclei of small grains with intense plastic deformations (Gholinia et. al., 2000). A higher scale level is represented by cell blocks that represent the volumes of adjacent cells. They have the same set of active slip systems, which causes their close orientation. The blocks are separated by dense dislocation boundaries and microbands (Fig. 1). Microbands are lamellar (long and thin) oriented regions, limited by the grain size. The width of the microbands is usually a few tenths of a micrometer, and the length is $0.1-10 \mu \mathrm{m}$. The boundaries of the blocks of cells are more clearly distinguished on microsections, and the misorientation of the blocks exceeds the misorientation of neighboring cells. The physically large misorientation of cell blocks (compared to neighboring cells) is explained by the different number of active slip systems in neighboring blocks. The boundaries of the microbands consist of dislocation substructures, similar to the walls of cells, but with a large content of dislocations. The crystallographic orientation of the microbands is close to adjacent cells and almost does not vary along the band (Gorelik et al., 2005). Nevertheless, in Hansen (1990) it is noted that in microbands there is a combination of slip systems that differ from the adjacent material. As plastic deformation occurs, a number of microbands and dense dislocation walls separating the original homogeneous grain of the polycrystal are increased. In the process of plastic deformation, the dimensions of the blocks of cells rapidly decrease, reaching one cell in the extreme case. Subsequent deformation leads to the appearance, on the larger-scale level, of domains, the number of which reaches 10-100 in the volume of grain (Kuhlmann-Wilsdorf and Hansen, 1991). Domains are divided among themselves by domain boundaries. The domains are the most disoriented scale elements, the sizes of which considerably exceed the sizes of cell 
blocks. In neighboring domains, as well as in cell blocks, there are various active slip systems, the number of which varies from three to five.

For three scale levels of the subgrain structure, three types of boundaries are distinguished: cell, cell block, and domain boundaries. All the marked boundaries are dislocations and have a low angle. The boundaries of the cell blocks and domains belong to geometrically necessary boundaries and are caused by rotations of the corresponding parts of the grain due to the motion and interaction of dislocations of various slip systems. For high-angle intergranular boundaries, which also belong to the geometrically necessary boundaries, evolution of the rotation of lattices of neighboring grains is also determined by the incompatibility of plastic deformations (Rybin, 1986). The boundaries of the cells differ from such boundaries. They are accidental, since they are formed as a result of trapping of edge mobile dislocations by barriers of various nature, including forest dislocations (Kuhlmann-Wilsdorf and Hansen, 1991). In the process of plastic deformation, the misorientation between adjacent cells increases in such a way that different slip systems begin to act in them. Consequently boundaries of cells evolve into geometrically necessary ones and become indistinguishable from the boundaries of blocks of cells and domains. In the work of Kuhlmann-Wilsdorf and Hansen (1991) such boundaries were called subgrain boundaries, with the elements separated by such boundaries being subgrains.

\section{THE MODEL FOR DESCRIBING LOW-ANGLE SUBGRAIN BOUNDARIES}

The problem of modeling the distribution of misorientations of the subgrain structure at the initial stage of plastic deformation is posed, when incidental cell boundaries are mainly formed. It is necessary to propose a method for modeling these boundaries, which can be used in the statistical model of inelastic deformation mentioned above, taking into account static recrystallization (Trusov and Kondratev, 2018), since for this model at the individual grain level the hypothesis about the uniformity of the stress-strain state is assumed. In this case, additional assumptions about the inhomogeneity of the plastic deformation of the polycrystalline grain should be introduced for the model of formation (appearance) of subgrain structure. According to the foregoing, it is assumed that incidental cell boundaries are formed as a result of the movement of edge dislocations along active systems and their trapping by various defects. Further, these boundaries evolve into the subgrain boundaries, increasing misorientation of the cells. Active slip systems for initially homogeneous grains are determined by the grain's crystallographic orientation with respect to the axes of the given loading. The hypothesis is accepted that during the formation of subgrains their shape, size, and crystallographic misorientation are independent model variables. On the basis of experimental data, the shape of the cells is approximated. At the initial stage of inelastic deformation, the

shape of the cell can be taken as hexagonal. The following procedure is used to set the orientation facet of cell boundaries. Cells are given a cubic form, which is ran-

Volume 9, Issue 4, 2018 


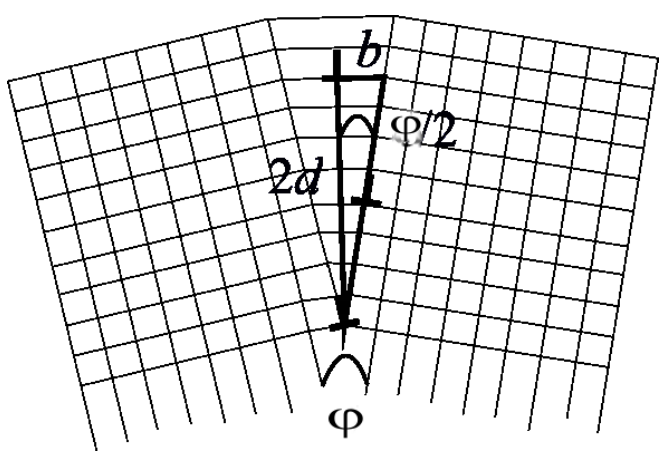

FIG. 2: Scheme for determining the misorientation angle of a subgrain

domly oriented in a uniform manner in space. Further, angles defining the orientation of the facet's normal in the crystallographic coordinate system are deflected randomly according to the uniform law within $10^{\circ}$ of the initial value. Before the completion of subgrains generation process, it is assumed that the crystallographic misorientation of lattices between subgrains can be neglected. The hypothesis is assumed that mobile edge dislocations on active slip systems accumulate in the form of walls in the boundaries of subgrains (Fig. 2). In this case, the small angle of misorientation $\varphi$ of neighboring subgrains (cells) can be assumed to be equal to (Burgers, 1940; Kuhlmann-Wilsdorf and Hansen, 1991; Friedel, 1964)

$$
\varphi=b / d,
$$

where $b$ is the modulus of the Burgers vector and $d$ is the distance between dislocations in the wall, a possible way of determining which is given below. For the case shown in Fig. 2, the direction of misorientation axis of the subgrain lattices coincides with the direction of the line of the considered edge dislocations $\mathbf{l}$. Next, it is necessary to obtain a ratio for the angle $\varphi$ using the internal parameters of the inelastic deformation model of the mesolevel (level of an individual grain) (Trusov and Kondratev, 2018).

Plastic deformation of polycrystalline grains is considered to be due to the motion of the edge dislocations along one $k$ th slip system. The magnitude of the inelastic shear $\gamma^{(k)}$ of the $k$ th slip system can be determined using the Orowan ratio:

$$
\gamma^{(k)}=\rho^{(k)} \lambda^{(k)} b
$$

where $\rho^{(k)}$ is the dislocation density on the $k$ th slip system and $\lambda^{(k)}$ is the dislocation mean free path on the $k$ th slip system. Before the beginning of plastic deformation, a hypothesis is used about the uniform distribution of dislocations in the grain volume (Fig. 3a). In this case, the density of dislocations in the forming subgrain $\rho_{c}^{(k)}$ and in the grain $\rho^{(k)}$ coincide. It is assumed that in the boundary's facet with the normal $\mathbf{N}^{(i)}$, the wall is built up only of dislocations interacting with this facet. 


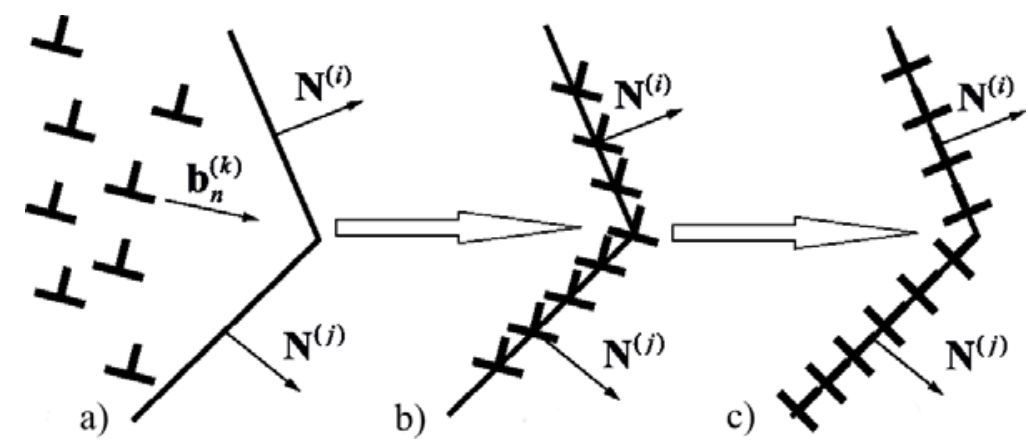

FIG. 3: The scheme for the formation of cell boundaries: a) the initial distribution of dislocations in the grain volume, b) the interaction of dislocations with the boundary, c) the separation from the dislocation cluster of the edge component lying in the boundary

In this case, only a certain fraction of the dislocation density of the $k$ th slip system of the subgrain, which has realized the considered shear $\gamma^{(k)}$, is in the dislocation wall of the facet $\mathbf{N}^{(i)}$. Figure $3 b$ shows the scheme of the "settling" part of the mobile dislocations of the $k$ th slip system on the $\mathbf{N}^{(i)}$ and $\mathbf{N}^{(j)}$ facets of the boundary. A measure of dislocations "settled" in the boundary is cosine of the angle $\alpha$ between the slip direction (the unit direction of the Burgers vector of the $k$ th system) and the outer normal to the facet of the boundary $\mathbf{N}^{(i)}$. In addition, it is necessary to take into account that only the fraction of dislocations $\beta$ "settles" on the boundary, with the remaining part of the dislocations being located in the inner regions of cells (Rybin, 1986). The parameter $\beta$ is determined on the basis of experimental studies as the ratio of the dislocation density in subgrain's boundaries to the total dislocation density in a grain. For the calculations described below, the parameter $\beta$ has the value 0.75 . Finally, the dislocation density $\rho_{w}^{(k)}$ "settles" in the boundary is determined by the relation

$$
\rho_{w}^{(k)}=\beta \rho_{c}^{(k)}\left(\mathbf{b}_{n}^{(k)} \cdot \mathbf{N}^{(i)}\right)=\beta \rho_{c}^{(k)} \cos (\alpha) .
$$

Further, for the "settled" density of dislocations of the kth slip system $\rho_{w}^{(k)}$, in the $\mathbf{N}^{(i)}$ facet the edge component of the dislocations lying in the facet under consideration is chosen (Fig. 3c). The Burgers vector of this component $\mathbf{b}_{d}^{(k)}$ is directed along the normal to the facet $\mathbf{N}^{(i)}: \mathbf{b}_{d}^{(k)}=\left(\mathbf{b}^{(k)} \cdot \mathbf{N}^{(i)}\right) \mathbf{N}^{(i)}$. The dislocation line vector $\mathbf{I}_{d}^{(k)}$ is directed along the line of intersection of the facet boundary plane $\mathbf{N}^{(i)}$ and the $k$ th slip plane $\mathbf{n}^{(k)}: \mathbf{I}_{d}^{(k)}=\mathbf{N}^{(i)} \times \mathbf{n}^{(k)}$. The third normal vector $\mathbf{n}_{d}^{(k)}$ is orthogonal to the first two: $\mathbf{n}_{d}^{(k)}=\mathbf{b}_{d}^{(k)} \times \mathbf{I}_{d}^{(k)}$. The ordered vector triples $\left(\mathbf{b}_{d}^{(k)}, \mathbf{n}_{d}^{(k)}, \mathbf{I}_{d}^{(k)}\right)$ are valid, the vectors $\mathbf{n}_{d}^{(k)}$ and $\mathbf{I}_{d}^{(k)}$ lie in the plane of the boundary (Fig. 4). As a result the dislocation wall with the edge component is singled out, as shown in Fig. 3c.

Only slip systems that interact with the facet $\mathbf{N}^{(i)}$ are considered, i.e., the condition is valid: $\mathbf{b}_{n}^{(k)} \cdot \mathbf{N}^{(i)}>0$. The model takes into account that the dislocations of the $k$ th slip system can move in the opposite direction. By definition, the disloca- 


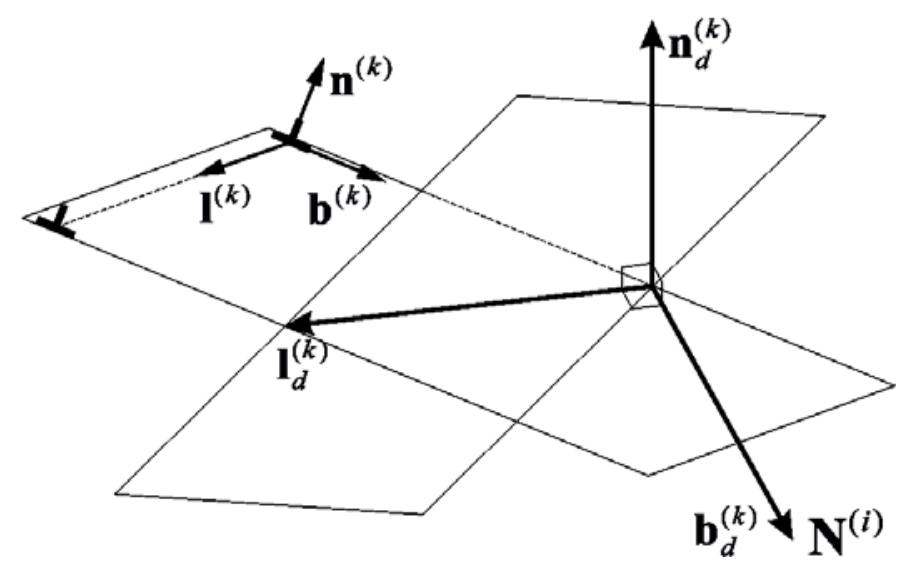

FIG. 4: The scheme for extracting the edge component basis of dislocations lying in the plane of the subgrain boundary $\mathbf{N}^{(k)}$

tion density $\rho_{w}^{(k)}$ is the magnitude of the total length of the dislocation lines $L_{w}^{(k)}$ per unit volume of the subgrain $V_{c}$ :

$$
\rho_{w}^{(k)}=\frac{L_{w}^{(k)}}{V_{c}} .
$$

Combining relations (2), (3), and (4), a relation for $L_{w}^{(k)}$ is obtained:

$$
L_{w}^{(k)}=\frac{\beta \gamma^{(k)} V_{c} \cos (\alpha)}{\lambda^{(k)} b} .
$$

Assuming that the current dimensions of the subgrain are known, the number of dislocation lines $N_{w}$ contained in the wall are determined (Fig. 5).

For this purpose, the ratio of the total length of dislocations $L_{w}^{(k)}$ to the length of the edge $l_{c}^{1}$ is found ( $l_{c}^{1}$ is the edge's length along which the wall is formed):

$$
N_{w}=\frac{L_{w}^{(k)}}{l_{c}^{1}}=\frac{\beta \gamma^{(k)} V_{c} \cos (\alpha)}{\lambda^{(k)} b l_{c}^{1}},
$$

then for a uniform distribution the distance between dislocation lines $d$ is equal to

$$
d=\frac{l_{c}^{2}}{N_{w}}=\frac{\lambda^{(k)} b l_{c}^{1} l_{c}^{(2)}}{\beta \gamma^{(k)} V_{c} \cos (\alpha)} .
$$




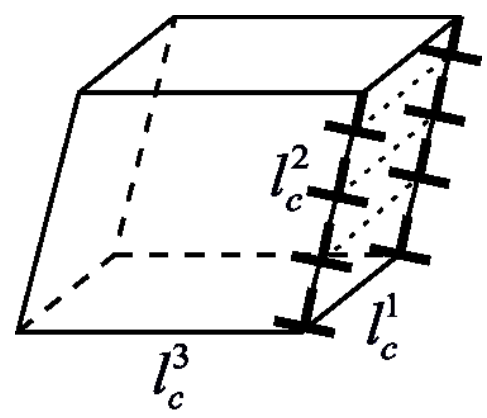

FIG 5: Scheme for formation of a dislocation wall in a subgrain boundary

For the deformation process, due to the slip of edge dislocations along the crystallite slip systems, the mean free path of the dislocation is comparable with the third direction of the subgrain $\lambda^{(k)} \approx l_{c}^{3}$ (Fig. 5). In this case, $V_{c} \approx \lambda^{(k)} l_{c}^{1} l_{c}^{2}$. Using (1), we finally obtain a relation for determining the misorientation angle of neighboring subgrains:

$$
\varphi^{(k)}=\beta \gamma^{(k)} \cos (\alpha)
$$

The shear magnitude $\gamma^{(k)}$ is determined using the mesolevel model (Trusov and Kondratev, 2018) for each active slip system that interacts with the subgrain boundary. The angle $\alpha$ is found using the initial data on the mutual orientation of the subgrain boundaries facets and the shear direction in the considered slip systems. Then the misorientation of the neighboring subgrains $\mathbf{0}_{s g}^{(k)}$, caused by the formation of the dislocation wall due to the motion of the $k$ th slip system, relative to the laboratory coordinate system, is determined by the relation

$$
\mathbf{0}_{s g}^{(k)}=\cos \left(\varphi_{s g}^{(k)}\right) \mathbf{I}+\left(1-\cos \left(\varphi_{s g}^{(k)}\right)\right) \mathbf{l}_{d}^{(k)} \mathbf{I}_{d}^{(k)}+\sin \left(\varphi_{s g}^{(k)}\right) \mathbf{l}_{d}^{(k)} \times \mathbf{I}
$$

where $\mathbf{I}$ is the unit tensor, the misorientation angle $\varphi_{s g}^{(k)}$ takes the values $\pm \varphi^{(k)} / 2$. The value of the misorientation angle $\varphi^{(k)} / 2$ with the plus sign is taken for the subgrain under consideration, minus for the neighboring (Fig. 2). As a result of the formed wall of dislocations, the mutual misorientation of neighboring subgrains will constitute an angle.

Since the orthogonal tensors do not represent a commutative group, for finding the final orientation for each subgrain the spin rotation of the crystallographic coordinate system is determined:

$$
\boldsymbol{\omega}=\sum_{k=1}^{N_{a}} \dot{\mathbf{o}}_{s g}^{(k)} \cdot\left(\mathbf{o}_{s g}^{(k)}\right)^{T} .
$$


where the summation is over all active $N_{a}$ slip systems that form dislocation walls on subgrain boundary facets, including the walls formed by the motion of dislocations in the neighboring subgrain. In relation (10), the index $T$ denotes the transpose of the tensor $\mathbf{0}_{s g}^{(k)}$. For the tensor $\omega$, the angular velocity vector $\mathbf{w}$ is determined: $\mathbf{w}=(1 / 2) \in: \omega$, where $\epsilon$ is the Levi-Civita tensor. The instantaneous axis of rotation e coincides with the direction of the vector $\mathbf{w}$ : $\mathbf{e}=\mathbf{w} / \sqrt{\mathbf{w} \cdot \mathbf{w}}$. The change in the angle of rotation of the subgrain lattice $\Delta \varphi$ during the time $\Delta t$ is determined by the length of the vector $\mathbf{w}: \Delta \varphi=(\sqrt{\mathbf{w} \cdot \mathbf{w}}) \Delta t$. For the known rotation axis $\mathbf{e}$ and the rotation angle $\Delta \varphi$, the lattice rotation tensor per step $\Delta \mathbf{o}_{s b}$ is determined using the relations of a similar (9). The tensor of the crystallographic orientation of the subgrain at the next instant $t+\Delta t \mathbf{0}_{s b}^{t+\Delta t}$ is defined as follows:

$$
\mathbf{0}_{s b}^{t+\Delta t}=\Delta \mathbf{o}_{s b} \cdot \mathbf{o}_{s b}^{t}
$$

where $\mathbf{0}_{s b}^{t}$ is the orientation tensor of the subgrain lattice at time $t$. In the initial configuration, it is assumed that the orientation of the subgrain coincides with the orientation of the grain.

\section{RESULTS OF NUMERICAL SIMULATION}

Numerical experiments were carried out to test the developed model of fragmentation. Using the two-level model of inelastic deformation (Trusov and Kondratev, 2018), uniaxial tension of copper polycrystal by a deformation amount of $11 \%$ is considered. The representative volume of a polycrystal consists of 1000 grains (each grain consists of 1000 subgrains), the crystallographic orientation of which is distributed according to the uniform law. Figure 6 shows the stress-strain intensity diagram (the $\sigma-\varepsilon$ diagram) for copper polycrystal and some random chosen polycrystalline grain. The diagram illustrates the initial stage of plastic deformation, which occurs due to the slip of dislocations along the primary system; linear strengthening of the material is observed.

Figure 7a shows the histogram for misorientation angles of the crystallographic coordinate system of subgrains to the corresponding grain, constructed from the results of model calculations and averaged across all the polycrystalline grains. Experimental data for misorientation of copper subgrains after $11 \%$ deformation by tension (Lebensohn et al., 2008) are presented in Fig. 7b. Figure 7c shows analogous data for one randomly chosen polycrystalline grain. As a result of the simulation, it is found that the average misorientation for subgrains in all polycrystal grains is $1.57^{\circ}$, with the maximum value being equal to $7.70^{\circ}$. In the experiment (Lebensohn et al., 2008) the average angle is $1.71^{\circ}$, the maximum angle is $6.62^{\circ}$. Numerical results obtained by the proposed model indicate a satisfactory quantitative agreement with the experimental data. In addition, it should be noted that the proposed model made it possible to describe not only the average characteristics of 


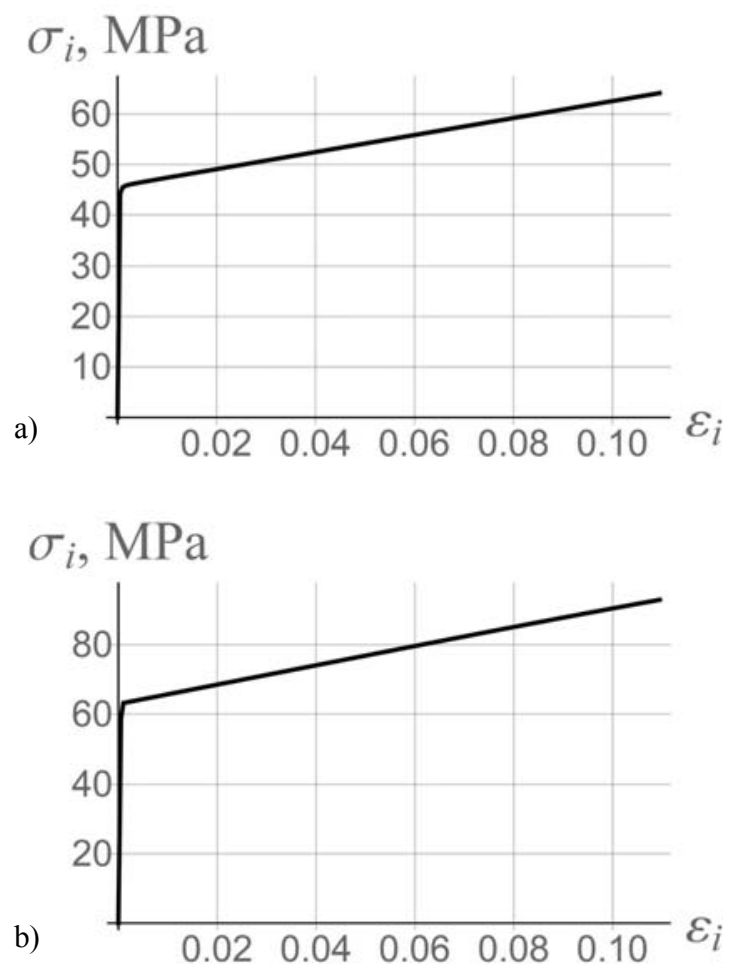

FIG. 6: The $\sigma-\varepsilon$ diagram of uniaxial tension: a) for copper polycrystalline; b) for randomly chosen polycrystalline grain

the misorientation angles, but also that the form of the misorientation angles distribution is close to the experimental one (Fig. 7).

The model allows one to determine the number of active slip systems in the formed cells. This can be useful in further analysis of the formation of blocks of cells. Blocks consist of cells that have the same set of active systems. This analysis is important, because it considers that the mechanism of recrystallization (Trusov and Kondratev, 2018) can also be realized both by the motion of cell boundaries and their blocks. Active slip systems were defined as follows. At the end of plastic deformation, shear rates were determined in all grains, their averaged value was found. In the case where the shear rate on any slip system was less than this value by 3 orders of magnitude, the system was considered not active. Figure 8 shows the histogram of active slip systems in the subgrains of the isolated grain at the end of deformation. The number of active slip systems in the original homogeneous grain (without taking into account the fragmentation model) is 6 , the subgrain rotations lead to a decrease in the number of active slip systems, which qualitatively agrees with the experimental data (Kuhlmann-Wilsdorf and Hansen, 1991). For the random grain at the end of plastic deformation, 6 active slip systems were in 705 subgrains, 5 were in 243,4 were in 52 (the average number of active slip 
a)

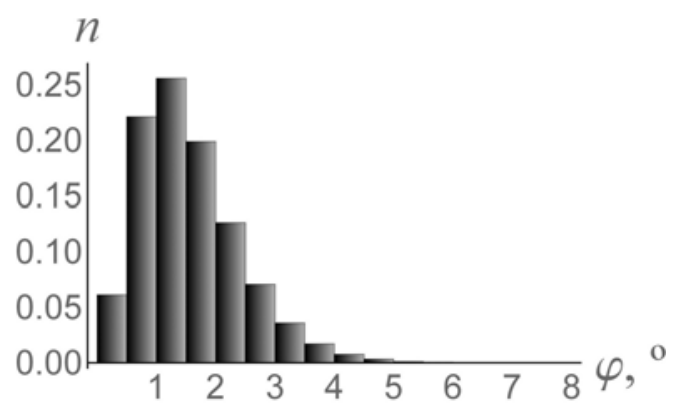

b)

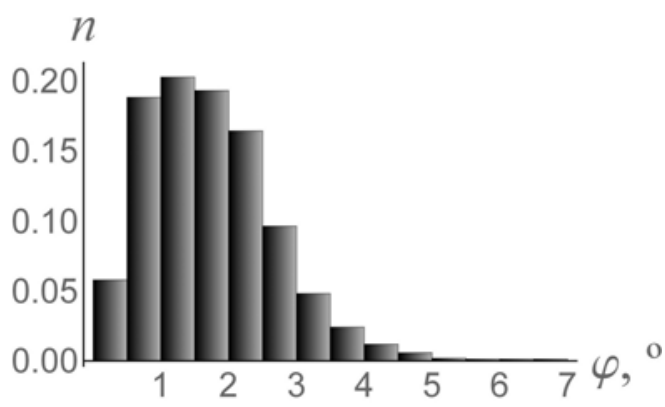

c)

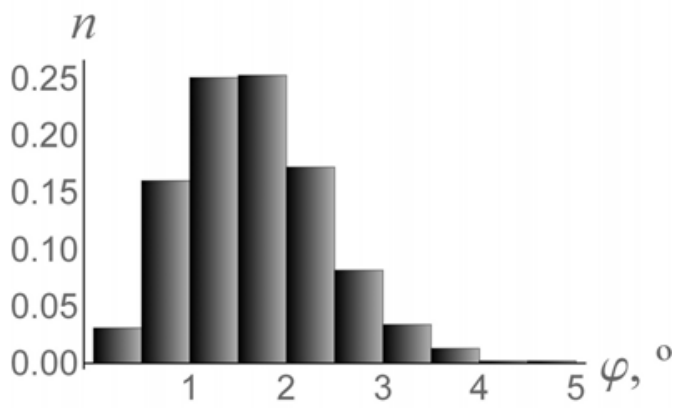

FIG. 7: Histograms for subrains intragranular misorientations of the crystallographic coordinate system: a) subgrains misorientations in all grains of the polycrystal, b) experimental data of subgrains misorientations, based on Lebensohn et al. (2008); c) subgrains misorientation of a randomly chosen polycrystalline grain

systems in subgrains was 5.653). The similar tendency to decrease the number of active slip systems is observed in all grains of a representative volume of a polycrystal. In the entire polycrystal, at the end of deformation number of active slip systems in homogeneous grains is 7.123, in subgrains is 7.079 .

The proposed method makes it possible to determine not only the angles of misorientation of subgrains but also the orientation of the crystallographic coordinate system of subgrains described by tensor (8) at different moments of deformation. Figure 9 shows a straight pole figure at the end of plastic deformation for a 


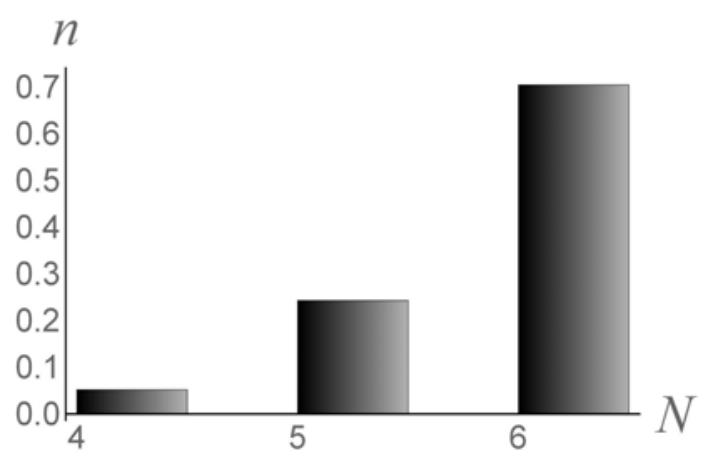

FIG. 8: Histogram of distribution of active slip systems in subgrains of isolated grain

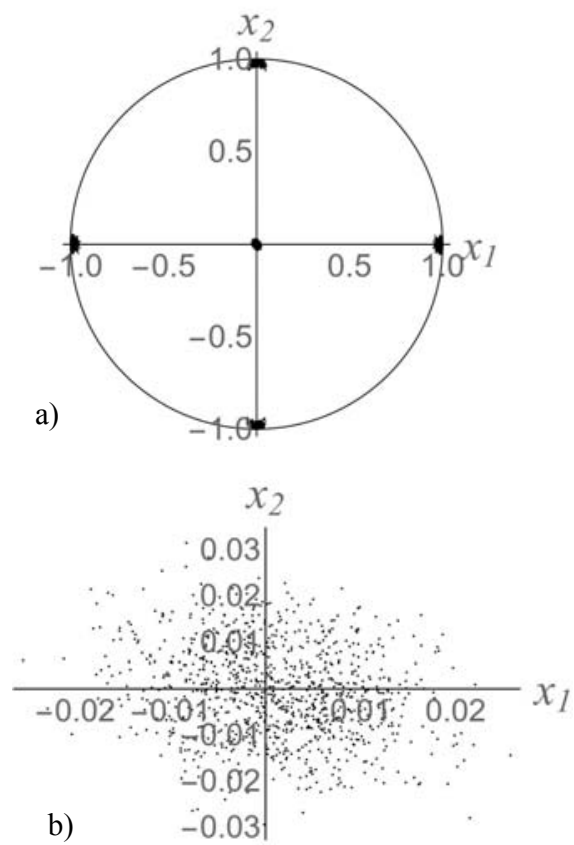

FIG. 9: The straight pole figure at the end of the deformation for the selected grain: a) for directions $\langle 100\rangle ; \mathrm{b})$ the enlarged image for the directions [001]

family of directions $\mathrm{d}$ of a randomly selected grain from a conglomerate constituting a polycrystal. The projection was carried out with respect to the [001] direction (the axis $\mathrm{Ox}_{3}$ is perpendicular to the plane of Fig. 9).

\section{CONCLUSIONS}

A model for determining the crystallographic orientation of subgrains is proposed, which is based on the formation of the physical mechanism of incidental cell 
boundaries. The shape of the cells is approximated by hexahedrons, facets orientation is randomly assigned according to the uniform law. At the initial stage of plastic deformation, it is assumed that cell boundaries are the main obstacle to the movement of edge dislocations. Dislocations are grouped into clusters near the boundaries (cells-dislocation walls), sequentially misorientation of the lattices of neighboring cells occurs. The mathematical description of the evolution of lowangle boundaries is proposed. It is shown that numerical results of subgrain misorientation distribution qualitatively and quantitatively coincide with the results of the experiments. The proposed method for modeling subgrain's misorientation describes the phenomenon of a decrease in the number of active slip systems as a result of subgrains rotations.

\section{ACKNOWLEDGMENTS}

Section 1 of the article was performed at the Perm National Research Polytechnic University, Sections 2-5 of the article were conducted at the Research Institute of Mechanics of the Lobachevsky State University, Nizhni Novgorod. The work was supported by the Russian Science Foundation (Project No. 15-19-10039).

\section{REFERENCES}

Bailey, J.E. and Hirsch, P.B., The Recrystallization Process in Some Polycrystalline Metals, Proc. Roy. Soc., vol. A267, pp. 11-30, 1962.

Burgers, J.M., Geometrical Considerations Concerning the Structural Irregularities to Be Assumed in a Crystal, Proc. Phys. Soc., vol. 52, no. 1, pp. 23-33, 1940.

Friedel, J., Dislocations, New York: Pergamon Press, 1964.

Gholinia, A., Prangnell, P.B., and Markushev, M.V., The Effect of Strain Path on the Development of Deformation Structures in Severely Deformed Aluminum Alloys Processed by ECAE, Acta Materialia, vol. 48, no. 5, pp. 1115-1130, 2000.

Gorelik, S.S., Dobatkin, S.V., and Kaputkina, L.M., Recrystallization of Metals and Alloys, Moscow: MISIS Press, 2005 (in Russian).

Gracio, J.J., Fernandes, J.V., and Schmitt, J.H., Effect of Grain Size on Substructural Evolution and Plastic Behavior of Copper, Mater. Sci. Eng.: A, vol. 118, pp. 97-105, 1989.

Hansen, N., Cold Deformation Microstructures, Mater. Sci. Technol., vol. 6, no. 11, pp. 1039-1047, 1990.

Koneva, N.A., The Nature of the Stages of Plastic Deformation, Soros Educational J., no. 10, pp. 99-105, 1998 (in Russian).

Koneva, N.A. and Kozlov, E.V., The Modern Picture of the Stages of Plastic Deformation, Izv. VUZov, Fiz., no. 8, pp. 90-98, 2004 (in Russian).

Kuhlmann-Wilsdorf, D., Theory of Plastic Deformation: Properties of Low Energy Dislocation Structures, Mater. Sci. Eng.: A, vol. 113, pp. 1-41, 1989.

Kuhlmann-Wilsdorf, D. and Hansen, N., Geometrically Necessary, Incidental and Subgrain Boundaries, Scripta Metallurgica et Materialia, vol. 25, no. 7, pp. 1557-1562, 1991.

Kuhlmann-Wilsdorf, D. and Thompson, A.W., Work Hardening in Tension and Fatigue, New York: AIME, 1977. 
Lebensohn, R.A., Brenner, R., Castelnau, O., and Rollett, A.D., Orientation Image-Based Micromechanical Modeling of Subgrain Texture Evolution in Polycrystalline Copper, Acta Materialia, vol. 56, no. 15, pp. 3914-3926, 2008.

Liu, Q. and Hansen, N., Deformation Microstructure and Orientation of FCC Crystals, Physica Status Solidi (a), vol. 149, no. 1, pp. 187-199, 1995.

Rybin, V.V., Large Plastic Deformation and Destruction of Metals, Moscow: Metallurgy Press, 1986 (in Russian).

Trusov, P.V. and Kondratev, N.S., Two-Level Elastoviscoplastic Model: Application to the Analysis of the Evolution of the Grain Structure under Static Recrystallization, Fiz. Mezomekh., vol. 21, no. 2, pp. 21-32, 2018 (in Russian).

Vishnyakov, Ya.D., Babarenko, A.A., Vladimirov, S.A., and Egiz, I.V., Theory of Texture Formation in Metals and Alloys, Moscow: Nauka Press, 1979 (in Russian).

Volume 9, Issue 4, 2018 



\title{
A VARIANT OF DESCRIBING ADHESION INTERACTION IN THE PROBE-SAMPLE SYSTEM OF AN ATOMIC-FORCE MICROSCOPE
}

\author{
V.Yu. Presnetsova, S.N. Romashin, L.Yu. Frolenkova, \\ V.S. Shorkin,* E S.I. Yakushina
}

I.S. Turgenev Orel State University, 95 Komsomolskaya Str., Orel, 302026, Russian Federation

*Address all correspondence to: V.S. Shorkin, I.S. Turgenev Orel State University, 95 Komsomolskaya Str., Orel, 302026, Russian Federation, E-mail: Vshorkin@yandex.ru

The paper presents a variant of describing the adhesion interaction in the probe-sample system of an atomic-force microscope constructed within the framework of the deformable solid mechanics. The variant is based on the concept of nonlocal pair and triple potential interactions of particles of a continuous elastic medium with account for the criteria of thermodynamicity and stability of both the discrete system of interacting material points and the system of infinitesimal particles constituting a continuous medium. In this case, the potentials of interaction of continuous medium particles are considered as the limits of analogous potentials of the interaction of particles forming an infinite sequence of discrete systems with distance between them, their masses, momenta, and energy tending to zero. The parameters of the interparticle potentials and the characteristics of the adhesive interaction between the sample and the probe are expressed in terms of the characteristics of the elastic state - Young's modulus and Poisson's ratio - under the assumption of their homogeneity, linear elasticity, and the isotropy of their materials. In order to verify the qualitative and quantitative adequacy, the model developed is used to calculate the dependence of the force of the van der Waals interaction between the probe (platinum) and the sample (graphite) on the distance between them. The results of calculation are in satisfactory agreement with the available data.

KEY WORDS: atomic-force microscope, solid elastic body, discrete system of material points, nonlocal many-particle interaction, thermodynamics, stability, adhesion

\section{INTRODUCTION}

Force spectroscopy by means of atomic-force microscopes is of considerable importance (Cappella and Dietler, 1999). However, the quantitative determination of the characteristics of materials by using them is not an easy task. This is due to many reasons, among which is the action of interaction forces between the probe and the sample (Dedkov et al., 2009), i.e., the adhesion forces. This paper is aimed at theoretical evaluation of the magnitude of the forces arising between the 
electrically neutral particles of the probe and sample (both during their contact and in its absence) by the methods of the mechanics of continuous elastic media. Such an assessment can substantially simplify the understanding and description of the results obtained in measurements.

A number of models can be used to describe the probe-sample interactions at the macroscopic level (Lifshitz, 1956; Hamaker, 1937; Derjaguin et al., 1975; Jonson et al., 1971; Maugis, 1992; Goryacheva and Makhovskaya, 2001; Lurie et al., 2018). Within the framework of these models, the interaction between the probe and the studied sample, and between their particles is a potential. The empirical functions characterizing these potentials contain the parameters to be determined experimentally, which complicates calculations.

The model used in this study allows one calculate adhesive interaction forces for samples and probes made of linearly elastic, homogeneous, isotropic materials on the basis of information on the elastic properties of the probe and sample materials. This approach can be applied to determine the potential parameters used in the models of Hamaker (1937), Derjaguin et al. (1975), Jonson et al. (1971), Maugis (1992), Goryacheva and Makhovskaya (2001), and Lurie et al. (2018).

The proposed model is based on the idea of the nonlocality of the interaction of solid-state particles. The concept of nonlocality is inherent in the solid-state physics, where nonlocal representations of atoms are abundant. Here the material is considered to consist of discretely distributed atoms - material points united into a single body by long-range forces from the neighboring atoms (Eringen, 2002; Born and Oppenheimer, 1927). The idea of nonlocal interaction typical of the solid-state physics, has found its use, for example, in Hamaker's theory of adhesive interaction of solids (Hamaker, 1937), in the method of molecular dynamics, and the method of particles (Hockney and Eastwood, 1981; Krivtsov, 2001). In constructing the model, the methods of statistical mechanics (Gibbs, 1982; Kubo, 1965; Ruelle, 1999) and the results of Shorkin (2011), Romashin et al. (2016), Frolenkova and Shorkin (2013), and Dolgov et al. (2015) were used.

\section{GENERAL CONCEPTS}

It is considered that any body $B$ (both the probe and the sample) is continuous, that is, represents a set of body-points $b$, at any instant of time $t$ homeomorphic to some regular geometric set of points $\Lambda_{t} \subset \mathfrak{R}^{3}$ (Truesdell, 1975; Zhilin, 2012), i.e., the actual or current configuration of the body $B$.

The body $B$ is considered to be isolated (first mentally at time $t=0$ by means of the surface $A_{0}$ and then actually at time $t=0+0$ ) from an infinitely extended continuous homogeneous isotropic linearly elastic medium $\Omega$. The configuration and state of the body $B$ at time $t=0$ are reference and thermodynamically equilibrium.

If $t>0$, the configuration and state are current. The two configurations of the body $B$ are considered equivalent, if the conversion of one of them into another is carried out by the motion that keeps the distance between the points. Therefore, 
the configurations $\Lambda_{t=0}$ and $\Lambda_{t=0+0}$ are considered equivalent, although the states of the material of the body $B$ in these configurations are not equivalent. The thermodynamic states of the material of the body $B$ in equivalent configurations are equivalent if the distributions of the parameters of these states coincide in the corresponding configurations. This assumption was introduced in order to reveal the effect of the apperance of surface energy (Maugis, 2000).

The position $b \in B$ in the reference and current configurations is characterized by the vectors $\vec{r}$ and $\vec{R}$. If $b_{i} \in B$ and $b_{j} \subset B$ are two arbitrary body-points with the numbers $i$ and $j$, then the position of the second point relative to the first one in these configurations is characterized by the vectors $\vec{l}_{i j}=\vec{r}_{j}-\vec{r}_{i}$ and $\vec{L}_{i j}=\vec{R}_{j}-\vec{R}_{i}=$ $\vec{l}_{i j}+\Delta \vec{u}_{i j}$. Here $\vec{u}=\vec{R}-\vec{r}$ is the displacement $b$ in transition from the position $\vec{r}$ to the position $\vec{R}$, and $\Delta \vec{u}_{i j}=\vec{u}_{j}-\vec{u}_{i}$ is the displacement $b_{j}$ relative to $b_{i}$.

The body-points $b$ are located in the centers of the inertia of infinitesimal particles $d B \subset B$. The kinematic characteristics of the body-points $b-$ their positions and velocities - are considered to be kinematic characteristics of $d B \subset B$. At any time, the infinitesimal particles $d B \equiv d B_{1}$ of the body $B$ interact with their own particles and of any other body through long-range potential forces. It is assumed that only pair and triple interactions are realized. The potential energy of the interacting particles is proportional to the product of their volumes:

$$
\begin{gathered}
d W_{t}^{(2)}=\Phi^{(2)}\left(\vartheta, \ldots, \theta, L_{12}(t)\right) d V_{t 1} d V_{t 2}, \\
d W_{t}^{(3)}=\Phi^{(3)}\left(\vartheta, \ldots, \theta, L_{12}(t), L_{13}(t)\right) d V_{t 1} d V_{t 2} d V_{t 3} .
\end{gathered}
$$

The proportionality coefficients $\Phi^{(2)} \equiv \Phi^{(2)}\left(\vartheta, \ldots, \theta, L_{12}\right)$ and $\Phi^{(3)}\left(\vartheta, \ldots, \theta, L_{12}, L_{13}\right)$ are the known functions of only (generally dependent on time) interparticle distances $L_{i j}=\left|\vec{L}_{i j}\right|=\left|\vec{R}_{j}-\vec{R}_{i}\right|$ due to the homogeneity and isotropy of the material. It is assumed that the form of these functions, the values of the parameters $\vartheta, \ldots, \theta$ that individualize the coefficients (further potentials) for specific materials, do not depend on either the configuration of the body or the location of the interacting particles inside it. The potential energy $d W_{t}(\vec{R})=w_{t}(\vec{R}) d V_{t}$ of the interaction of the particle $d B \equiv d B_{1}$ with other particles of the body $B$ and the force $d \vec{F}_{t}(\vec{R})=\vec{f}_{t}(\vec{R}) d V_{t}$ acting on the particle from the environment are defined by the equations

$$
\begin{gathered}
d W_{t}=w(\vec{R}) d V_{t}=\left\{\int_{\Lambda_{t}} \Phi^{(2)}\left(L_{12}\right) d V_{t 2}+\int_{\Lambda_{t}} d V_{t 2} \int_{\Lambda_{t}} \Phi^{(3)}\left(L_{12}, L_{13}\right) d V_{t 3}\right\} d V_{t}, \\
d \vec{F}_{t}(\vec{R})=\vec{f}_{t}(\vec{R}) d V_{t}=-\nabla_{\vec{R}} w_{t}(\vec{R}) d V_{t}, \quad\left(\nabla_{\vec{R}} \equiv \frac{d}{d \vec{R}}\right) .
\end{gathered}
$$

Account for only pair interactions is widely used in the study of adhesion problems, starting from the Hamaker model (Hamaker, 1937). At present, triple particle 
interactions are also taken into account to refine the results obtained (Frolenkova and Shorkin, 2017).

Suppose that at the time $t=0$ the body $B$ is separated mentally in an infinite medium $\Omega$. At this moment, the distributions of the temperature $T_{0}$ and of the material density $\rho_{0}$ over the area $\Lambda_{0} \equiv \Lambda \subset \mathfrak{R}^{3}$ occupied by the body $B$ are uniform. The quantity $w(\vec{r}) \equiv w(\vec{r}, t=0)$ is determined by the equality obtained from (1) by replacing $\Lambda_{t}$ by $\mathfrak{R}^{3}$, hence, it is also distributed uniformly over $\Lambda: w(\vec{r}, t=0)=w_{0}=$ const. Therefore, the vector $\vec{f}(\vec{r})=-\nabla_{\vec{r}} w(\vec{r}, t=0)=0$.

Hence, all the particles $d B \subset B$ are in equilibrium. In this case, the internal potential energy of the body $B$ is determined by the equality

$$
W_{0}=\int_{\Lambda} w(\vec{r}) d V=w_{0} V .
$$

At the time $t=0+0$, the work $G$ of instantaneous separation of the body $B$ from the infinitely extended medium $\Omega$ is performed with the same distributions of $\rho$, $T_{0}$, and $w_{0}$ as in the body $B$. At this moment, the interaction of the particles $d B \subset B$ with the particles $d C \subset C \subset \Omega \backslash B$ in the addition to the body $B$ in the framework of $\Omega$ breaks, with the interactions of the particles $d B \subset B$ between themselves being preserved. The instantaneous separation means the constancy of the positions of the particles $d B \subset B$ (as before, $\vec{R}=\vec{r}$ ).

For these reasons

$$
\vec{f}(\vec{r})=-\nabla_{\vec{r}} w(\vec{r}, \quad t=0+0) \neq 0 .
$$

The change in the thermal energy accompanied by thermal expansion does not occur because of the instantaneous separation. Therefore the work $G$ is equal to the change in the potential energy of the body $B$ :

$$
\begin{gathered}
G=\int_{\Lambda}[w(\vec{r}, t=0+0)-(\vec{r}, t=0)] d V \\
=W(t=0+0)-W(t=0)=\Delta W .
\end{gathered}
$$

The value of $G$ is equal to the work done to form the real surface $A_{0}$ of the body $B$, that is, its surface energy at the time $t=0+0$.

Due to the originated instability of the internal forces in the isolated body $B$, deformations and internal stresses arise in it that in the absence of heat exchange with the medium in which the body $B$ occurs will entail a change in the temperature distribution. As a result, a uniform distribution of the new temperature $T \neq T_{0}$ will appear with time, as well as the distribution of the internal potential 
energy with bulk density $w(\vec{R})$, not coinciding with $w(\vec{r}=0+0)$, when $\vec{f}(\vec{R})=0$. The configuration of the region occupied by the body $B$ from $\Lambda$ to $\Lambda_{t}$ will change. It is obvious that in this case it will turn out that

$$
G \neq \int_{\Lambda_{t}} w(\vec{R}) d V_{t}-\int_{\Lambda} w(\vec{r}, t=0) d V=\Delta_{t} W .
$$

It is further assumed that the deformations developing because of the appearance of forces (5) are so small that the change in the configuration in calculating the integrals, gradients, and elementary volumes entering into expressions (2), (3), and (7) and the changes in the temperature and density of materials caused by them can be neglected and it can be considered that

$$
\left|G-\Delta_{t} W\right|=0, \quad G=\Delta_{t} W=\Delta W
$$

In accordance with Lifshitz (1956) and Hamaker (1937), the adhesive interaction of solids begins even when the distance between them is not equal to zero. The sticking effect, called adhesion, is the final stage of this interaction.

Let $B_{(\alpha)}(\alpha=1,2)$ consist of two bodies for which adhesive interaction is to be considered. The relative position of their configurations $\Lambda_{(\alpha) t} \subset \mathfrak{R}^{3}$ is characterized by the vector $\vec{\alpha}_{t}=\vec{O}(1) t_{O_{(2) t}}=\vec{e}_{a t} a_{t}$ which connects the centers of inertia of the bodies $B_{(\alpha)}$, and by the relative orientation of the trihedrals $\vec{e}_{(\alpha) i}$ formed by the unit vectors of the inertia axes of the bodies $B_{(\alpha)}$.

The position of the particles $d B_{(\alpha)}$ is assigned relative to the centers of inertia $O_{(\alpha) t}$ of the sample particles $d B_{0(\alpha)}$ in the current configuration by their radius vectors $\vec{R}_{(\alpha)}$ and by $\vec{r}_{(\alpha)}$ in the reference configuration. The relative position of the particles of different bodies in these configurations of the system

$$
B=\bigcup_{\alpha=1}^{2} B_{(\alpha)}
$$

is assigned by the vectors $\vec{L}_{(\alpha \beta)}$ and $\vec{l}_{(\alpha \beta)}$.

The vector $\vec{h}_{t}=\overrightarrow{M_{(1) t} M_{(2)} t}=\vec{e}_{h t} h_{t}\left(\left|\vec{e}_{h t}\right|=1\right)$ that connects two fixed particles $d B_{A \text { (1) }}$ and $d B_{A \text { (2) }}$ with the centers at the points $M_{(1) t}$ and $M_{(2) t}$ of the boundary surfaces $A_{(1) t}$ and $A_{(2) t}$ is also given. The distance $\Phi_{(N)}^{(2)}$ between them is taken as the distance between the bodies $B_{(1)}$ and $B_{(2)}$, whereas the direction of the vector $\vec{e}_{h t}$ is taken to be the direction, in which the action of the adhesive forces is of interest.

It is admitted that the long-range interaction is characteristic not only for particles $d B_{(\alpha) i}$ of one body $B \equiv B_{(\alpha)}$, but also for the particles $d B_{(1) i}$ and $d B_{(2) j}$ from different bodies $B_{(1)}$ and $B_{(2)}$. In this case, the energy $d W_{(\alpha)}=w_{(\alpha) t} d V_{(\alpha) t}(\alpha=1,2)$ of 
the particle $d B_{(\alpha)} \equiv d B_{(\alpha) 1}$ is equal to the sum of the energies $w_{(\alpha \alpha) t} d V_{(\alpha) t}$ of its interactions inside $B_{(\alpha)}$ and of the energies $w_{(\alpha \beta) t} d V_{(\alpha) t}(\alpha, \beta=1,2 ; \alpha \neq \beta)$ of those of its interactions, in which the particles of the body $B_{(\beta)}$ participate.

$$
w_{(\alpha) t}=w_{(\alpha \alpha) t}+w_{(\alpha \beta) t} .
$$

The volume densities $w_{(\alpha \alpha) t}$ and $w_{(\alpha \beta)}$ of these types of energy are determined by the equalities

$$
\begin{gathered}
w_{(\alpha \alpha) t}=\int_{V_{(\alpha)}} \Phi_{(\alpha \alpha) t}^{(2)} d V_{(\alpha) t 2}+\int_{V_{(1)}} d V_{(\alpha) t 2} \int_{V_{(\alpha)}} \Phi_{(\alpha \alpha \alpha) t}^{(3)} d V_{(\alpha) t 3} \\
w_{(\alpha \beta) t}=\int_{V_{(\beta)}} \Phi_{(\alpha \beta) t}^{(2)} d V_{(\beta) t 2}+\int_{V_{(\alpha)}} d V_{(\alpha) t 2} \int_{V_{(2)}} \Phi_{(\alpha \alpha \beta) t}^{(3)} d V_{(\beta) t 3}+\int_{V_{(\beta)}} d V_{(\beta) t 2} \int_{V_{(\beta)}} \Phi_{(\alpha \beta \beta) t}^{(3)} d V_{(\beta) t 3}
\end{gathered}
$$

An arbitrary particle $d B_{(\alpha)} \equiv d B_{(\alpha)} 1$ of the body $B_{(\alpha)}$ turns out to be subject to the action of the force $d \vec{F}_{(\alpha) t}=\vec{f}_{(\alpha) t} d V_{(\alpha) t}=-\nabla_{\vec{R}} w_{(\alpha) t} d V_{t}$ with a bulk density $\vec{f}_{(\alpha) t}=\vec{f}_{(\alpha \alpha) t}+\vec{f}_{(\alpha \beta) t}+\vec{\phi}_{(\alpha) t}$ (not summable over $\alpha$ ).

The term $\vec{f}_{(\alpha \alpha) t}=-\nabla_{\vec{R}} w_{(\alpha \alpha) t}$ is the volume density of the forces acting on the particle $d B_{(\alpha)} \equiv d B_{(\alpha) 1}$ of the body $B_{(\alpha)}$ from the side of the other particles of the same body $B_{(\alpha)}$; the term $\vec{f}_{(\alpha \beta) t}=-\nabla_{\vec{R}} w_{(\alpha \beta) t}$ is the volume density of the forces acting on $d B_{(\alpha)}$ with participation of the particles of the body $B_{(\beta)}$ : in pair interactions, only of the particles of the body $B_{(\beta)}$, and in triple interactions (depending on the type of particles forming the interacting triplet), both with the participation of the particles from $B_{(\beta)}$ and from $\Phi_{(N)}^{(2)}$; the term $\vec{\phi}_{(\alpha) t}$ is the volume density of the forces acting from the side of the environment of both bodies $B_{(\alpha)}$ and $B_{(\beta)}$.

We believe that there are no other types of impacts on the particles of the interacting bodies $B_{(1)}$ and $B_{(2)}$; for the particles of each of them, we can write the equations of motion:

$$
\begin{aligned}
& \rho_{(1) t} \ddot{\vec{u}}_{(1)}=\vec{f}_{(11) t}+\vec{f}_{(12) t}+\vec{\phi}_{(1) t}, \\
& \rho_{(2)} \ddot{\vec{u}}{ }_{(2)}=\vec{f}_{(22) t}+\vec{f}_{(21) t}+\vec{\phi}_{(2) t} .
\end{aligned}
$$

The system of equations (12) must be supplemented with the initial conditions. The vectors $\Phi_{(N)}^{(2)}$ entering into the right-hand sides of Eqs. (12) are defined in terms of the potentials $\Phi_{(\alpha \beta)}^{(2)}$ and $\Phi_{(\alpha \beta \gamma)}^{(2)}$ of the pair and triple interactions of particles of both one body and different bodies. The arguments of these potentials are the vectors $\vec{l}_{(\alpha) 1(\beta) j}$ of the relative positions of interacting particles in the reference state and the vectors of the relative displacements $\vec{u}_{(\alpha) 1(\beta) j}$. The materials of interacting bodies are considered homogeneous and isotropic. Therefore these dependences have the form:

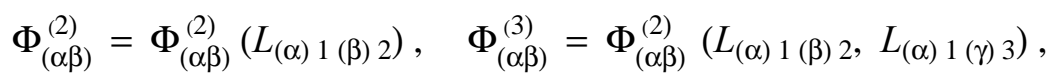


where $L_{(\alpha \beta)}$ is the absolute value of the vector $\vec{L}_{(\alpha) 1(\beta) j}=\vec{l}_{(\alpha) 1(\beta) j}+\vec{u}_{(\alpha) 1(\beta) j}(\alpha ; \beta$, $\gamma=1,2$; the equality between the indices is possible). These functional dependences are considered known and corresponding to the stability condition of the system of particles, the interaction of which is described with their help and with the vanishing at infinity. They can be calculated, if the fields $\vec{u}_{(\alpha) 1(\beta) j}$ and the values of those parameters that enter into the dependences that individualize them for each material and each pair of interacting materials are known. In the physics and mechanics of discrete structures, these parameters are determined on the basis of experiments or additional physical studies. This very procedure is proposed in the present paper.

The system of equations (12) with known initial conditions describes the motion of the system $B=B_{(1)} \cup B_{(2)}$ under the action of internal adhesive and external volumetrically distributed forces. Based on the solution of this problem with the help of Eqs. (9)-(11) and definitions

$$
\begin{gathered}
\vec{f}_{(\alpha \beta) t}=-\nabla_{\vec{R}} w_{(\alpha \beta) t}, \\
\vec{F}_{(\alpha \beta) t}=\int_{\Lambda_{(\beta) t}} \vec{f}_{(\alpha \beta) t} d V_{(\beta) t}=-\vec{F}_{(\beta \alpha) t}=-\int_{\Lambda_{(\alpha) t}} \vec{f}_{(\beta \alpha) t} d V_{(\alpha) t},
\end{gathered}
$$

we calculate the force $\vec{F}_{h(\alpha \beta)}=\vec{e}_{h}\left(\vec{e}_{h} \cdot \vec{F}_{(\alpha \beta)}\right)$ of the adhesive influence on the body $B_{(\alpha)}$ from the side of the body $B_{(\beta)}$ located in a given manner relative to $B_{(\alpha)}$ under the conditions of the external field $\vec{\phi}_{(\alpha) t}$. Using the solution obtained, we can then find the dependence of this force on the vector $\vec{h}$ and on the mutual orientation of the bodies $B_{(\alpha)}$ and $B_{(\beta)}$. The complexity of the solution of this problem in a general form is obvious, although there are particular solutions of similar problems (Ivanova et al., 2007; Berinskii et al., 2007; Grekova, 1996; Byzov and Ivanova, 2007).

We suggest the following variant. The properties of homogeneity and isotropy are not characteristic for the surface layers of solids. Therefore, it is admitted that the bodies $B_{(\alpha)}$ are separated instantly, at the same time $t=0+0$, from the depths of infinitely extended media $\Omega_{(\alpha)}$ and are placed in the region $\Lambda_{0-0(\alpha)} \equiv \Lambda_{(\alpha)} \subset \mathfrak{R}^{3}$. These regions are the configurations of the bodies $B_{(\alpha)}$ equivalent to their initial configurations $\Lambda_{0(\alpha)} \subset \mathfrak{R}^{3}$ when the bodies $B_{(\alpha)}$ were isolated in $\Omega_{(\alpha)}$ only mentally at the moment $t=0$. The equivalence of the configurations means that transition from one into another for each of the bodies has not changed the interparticle distances inside them. The mutual orientation of the regions and the vector $\vec{h}$ for them are considered given, with the temperatures of the bodies being equal to one another.

The hypotheses for the isolated bodies introduced when analyzing the processes accompanying the isolation of one body are considered valid. As soon as the bodies $B_{(\alpha)}$ occupy the positions $\Lambda_{(\alpha)}$, the initial positions of the particles $d B_{(\beta) j}$ with respect to each particle $B_{(\alpha)} \equiv d B_{(\alpha) 1}$ become known: $\vec{l}_{(\alpha) 1(\beta) j}=\vec{a}_{(\alpha \beta)}+\left(\vec{r}_{(\beta) j}-\vec{r}_{(\alpha) 1}\right)$. 
The instantaneous occurrence of bodies in the configuration $\Lambda_{(\alpha)}$ and, accordingly, the instantaneous entrance into the sphere of action of interparticle forces lead to the violation of the internal equilibria and to the start of the transient processes with changes of temperatures and relative positions of the interacting particles. Therefore, in the presence of energy exchange with the environment, the initial total potential energy may undergo a change with time.

It is assumed that the relative displacements of particles $d B_{(\alpha)}$ and $d B_{(\beta)}$ caused by deformations of the bodies $B_{(\alpha)}$ and $B_{(\beta)}$ because of the instability of their internal forces originated after their instantaneous separation and also their instantaneous occurrence in the sphere of mutual influence are small in comparison with $h=|\vec{h}|$. It is also supposed that external (relative to the system $B=B_{(1)} \cup B_{(2)}$ ) effects on it are absent $\left(\vec{\phi}_{(\alpha)}=\overrightarrow{0}\right)$.

In this case, the energy losses during the transition from the nonequilibrium state of the system

$$
B=\bigcup_{\alpha=1}^{2} B_{(\alpha)}
$$

to its equilibrium state can be neglected, and we can assume that the energy of the adhesion interaction of two bodies $B_{(\alpha)}$ and $B_{(\beta)}$, located in a special way relative to one another depending on the chosen distance $h$ between the fixed points of their boundaries, is determined by the expression

$$
W_{(\alpha \beta)}(h)=\int_{\Lambda_{(\beta)}} w_{(\alpha \beta)}(h) d V_{(\beta)} .
$$

In this expression, the integrand is determined by expression (11) written for the instant of time $t=0+0$, when the relative displacements of the particles and the changes in the configurations of the interacting bodies are equal to zero.

The magnitude of the interaction force in the direction of the vector $\vec{h}=\vec{e}_{h} h$ can be determined in this case with account for the fact that the regions $\Lambda_{(\alpha)}$ and $\Lambda_{(\beta)}$ do not change with variation of the value of $h$. That is, it is assumed that

$$
\vec{F}_{(\alpha \beta)}=\left[\vec{F}_{(\alpha \beta) t}\right]_{t=0+0}=\int_{\Lambda_{(\beta)}} \vec{f}_{(\alpha \beta)} d V_{(\beta)}
$$

Then

$$
\vec{F}_{(\alpha \beta) h}=\vec{e}_{h} F_{(\alpha \beta) h},
$$




$$
\begin{aligned}
F_{(\alpha \beta) h} & =-\int_{\Lambda_{(\beta)}} \vec{e}_{h} \cdot\left(\nabla_{\vec{r}} w_{(\alpha \beta)}\right) d V_{(\beta)}=-\int_{\Lambda_{(\beta)}} \frac{d w_{(\alpha \beta)}}{d h} d V_{(\beta)} \\
& =-\frac{d}{d h} \int_{\Lambda_{(\beta)}} w_{(\alpha \beta)} d V_{(\beta)}=-\frac{d}{d h} W_{(\alpha \beta)}(h) .
\end{aligned}
$$

As a result, it turns out that the magnitude and sign (with respect to the direction $\vec{e}_{h}$ ) of the force of the adhesion interaction of two bodies with invariable mutual orientation is determined as the derivative in this direction.

Further arguments concern the determination of the potentials of pair and triple interactions of particles of a homogeneous continuous medium on the basis of the notions used in the description of discrete media.

When a continuum approach is used on the set $\Lambda_{t}$ that is occupied by the solid body $B$, its measure (volume $V_{t}$ ) is given at the time $t>0$. On the set $B$ of bodypoints $b$, its measure (mass $m_{t}$ ) is given. In addition, the body $B$ has an impulse $\vec{P}$ and potential energy $W_{t}$ of interaction of its parts and their mechanical interaction with the environment.

It is supposed (Palmov, 2008) that the properties of large parts of a deformable body do not change when they are crushed into arbitrarily small parts. This allows one to introduce the density $\rho_{t}$ of the distribution of mass, impulse $\vec{p}_{t}$, and energy $w_{t}$ :

$$
\begin{gathered}
\left(m_{t}, \vec{P}_{t}, W_{t}\right)=\sum_{n=1}^{N}\left(\Delta m_{t}, \Delta \vec{P}_{t}, \Delta W_{t}\right) \\
=\lim _{N \rightarrow \infty, \Delta V_{t} \rightarrow 0} \sum_{n=1}^{N}\left(\rho_{t}, \vec{p}_{t}, w_{t}\right)\left(M_{n}\right) \Delta V_{t}=\int_{\Lambda_{t}}\left(\rho_{t}, \vec{p}_{t}, w_{t}\right)\left(M_{n}\right) d V_{t} .
\end{gathered}
$$

The integral sums in (19) were obtained in mental division of the body $B$ into $N$ parts $\Delta B_{(N) n}$ and in bringing their masses, impulses, and energies to the centers of inertia $M_{n}(t)$ with their subsequent summation. It turns out that these characteristics of a continuous body are the limits of the sequences of the corresponding characteristics of discrete systems $B_{N}$ with an infinitely increasing number of material points and with the distance between them that tends to zero. These systems at any time occupy the same region $\Lambda_{t} \subset \mathfrak{R}^{3}$ as the solid body.

Usually, if the medium is discrete, then its particles are considered as material body-points that directly model atoms or molecules of the real structure of the material (Born and Huang, 1954; Gusev and Lurie, 2010; Lurie and Solyaev, 2017). The elements of the structures used by the particle dynamics method (Hockney and Eastwood, 1981; Kittle, 1975) can be considered as body-points.

For each continuous body $B$ we set a sequence of discrete structures $\widehat{B}=\left\{\widehat{B}^{(N)}\right\}$ with such distributions of material points $b_{n}^{(N)} \in \widehat{B}^{(N)}$ over the integration region $\Lambda_{t}$ that coincide with the positions of the centers of inertia of the elements $\Delta B_{(N)} n$. 
They are obtained by dividing the body $B$ into $N$ parts when constructing the integral sums in calculating the mass, momentum, or energy. The material points $b_{n}^{(N)} \in \widehat{B}^{(N)}$ have masses, impulses, and energy of the parts $\Delta B_{(N) n}$. For this system, the region $\Lambda_{t}$ is assumed limited, Lebesgue measurable, with $V_{t}$ being its Lebesgue measure.

For each discrete system $\widehat{B}^{(N)}$, it is admitted that the impulses of its particles $(\Delta \vec{P}) \underset{(n) t}{(N)}$ are random quantities that can take any values - from zero to infinity and any directions. The values of the volume densities $\vec{p}_{t}$ of the impulses $d \vec{P}_{t}$ of infinitesimal particles $d B \subset B$ of a continuous body $B$ are obtained by averaging the possible impulses $(\Delta \vec{P})^{(N)}$ with the subsequent limiting transition

$$
\vec{p}_{t}=\lim _{n \rightarrow \infty} \frac{(\Delta \vec{P})_{(n) t}^{(N)}}{(\Delta V)_{(n) t}^{(N)}} .
$$

Just as for the body $B$, so for any of its discrete approximation $\widehat{B}^{(N)}$, we can write the expression for the Hamiltonian:

$$
\begin{gathered}
H_{t}=\int_{\Lambda_{t}} \frac{\vec{p}_{t}^{2}}{2 \rho_{t}} d V_{t}+\int_{\Lambda_{t}} w_{t} d V_{t} \\
H_{(N) t}=\sum_{n=1}^{N} \frac{\vec{p}_{(n) t}^{2}}{2 \rho_{(n) t}} \Delta V_{(n) t}+\sum_{n=1}^{N} w_{(n) t} \Delta V_{(n) t}=\sum_{n=1}^{N} \frac{(\Delta \vec{P})_{(n) t}^{2}}{2 \Delta m_{(n) t}}+W_{(N) t}\left(\vec{R}_{1}, \ldots, \vec{R}_{N}\right) .
\end{gathered}
$$

In expression (21), the quantity $W_{(N) t}\left(\vec{R}_{1}, \ldots, \vec{R}_{N}\right)$ is the energy of the potential interaction of particles $b_{n}^{(N)}$ among themselves and with the surrounding medium. This energy can be presented as a sum of potential energies of interaction of particles $b_{n}^{(N)}$ with their environment both inside the system and outside it. As for any discrete system (Ruelle, 1999), the energy $W_{(N) t}\left(\vec{R}_{1}, \ldots, \vec{R}_{N}\right)$ can be represented as the sum of the energies of individual particles $\Delta W_{n(N) t}^{(1)}\left(\vec{R}_{1}\right)$ (it plays the role of the chemical potential, it can be the energy of their elastic deformations), of the interaction of their pairs $\Delta W_{n(N) t}^{(2)}\left(\vec{R}_{1}, \vec{R}_{2}\right)$, triples $\Delta W_{n(N) t}^{(3)}\left(\vec{R}_{1}, \vec{R}_{2}, \vec{R}_{3}\right)$, etc. In the work, not more than triple interactions are taken into account:

$$
\Delta W_{n(N) t}^{(k)}\left(\vec{R}_{1}, \ldots, \vec{R}_{k}\right)=w_{n(N) t}^{(k)}\left(\vec{R}_{1}, \ldots, \vec{R}_{k}\right) \Delta V_{1(N) t} \ldots \Delta V_{n(N) t}^{(k)} .
$$

It is obvious that

$$
H_{t}=\lim _{N \rightarrow \infty, \Delta V_{t} \rightarrow 0} H_{(N) t}
$$

To the system $B=B_{(1)} \cup B_{(2)}$ of bodies $B_{(\alpha)}(\alpha=1,2)$ that experience adhesion interaction there corresponds the discrete structure 


$$
\widehat{B}^{(N)}=\widehat{B}_{(1)}^{(N)} \bigcup \widehat{B}_{(2)}^{(N)}\left(N=N_{(1)}+N_{(2)}\right) .
$$

For this system there corresponds the Hamiltonian

$$
H_{(N) t}=\sum_{\alpha=1}^{2} \sum_{n=1}^{N} \frac{(\Delta \vec{P})_{(n(\alpha) t}^{2}}{2 \Delta m_{(n(\alpha) t}}+W_{(N) t} .
$$

Its limit is the Hamiltonian of the system $B=B_{(1)} \cup B_{(2)}$ of continuous bodies:

$$
H_{t}=\lim _{\substack{N(1) \rightarrow \infty, \Delta V_{(1) t \rightarrow 0,} \\ N(2) \rightarrow \infty, \Delta V_{(2) t \rightarrow 0}}} H_{(N) t} .
$$

In accordance with Krivtsov (2001), the potential energy $W_{(N) t}$ of the system

$$
\widehat{B}^{(N)}=\widehat{B}_{(1)}^{(N)} \bigcup \widehat{B}_{(2)}^{(N)}
$$

is the sum of the potential energies of their parts $\widehat{B}_{(\alpha)}^{(N)}$ and of the energy $W_{N(12)}$ of their interaction, which is taken as the energy of their adhesion:

$$
\begin{gathered}
W_{(N) t}=W_{(N) t}\left(\vec{R}_{1(1)}, \ldots, \vec{R}_{N(1)} ; \vec{R}_{1(2)}, \ldots, \vec{R}_{N(2)}\right)=\sum_{\alpha=1}^{2} W_{N(\alpha) t}\left(\vec{R}_{1(\alpha)}, \ldots, \vec{R}_{N(\alpha)}\right) \\
+W_{N(12) t}\left(\vec{R}_{1(1)}, \ldots, \vec{R}_{N(1)} ; \vec{R}_{1(2)}, \ldots, \vec{R}_{N(2)}\right) .
\end{gathered}
$$

Just as for an individual body, the energy (26) is represented as a sum of energies of not more than triple interactions:

$$
\begin{aligned}
& W_{(N) t}=\sum_{\alpha=1}^{2}\left(\sum_{n=1} \Delta W_{n(\alpha) t}^{(1)}+\sum_{m>n} \sum_{n} \Delta W_{n m(\alpha) t}^{(2)}+\sum_{p>m>n} \sum_{m>n} \sum_{n} \Delta W_{n m p(\alpha) t}^{(3)}\right) \\
& +\left(\sum_{m(2)=1}^{N(2)} \sum_{n(1)=1}^{N(1)} \Delta W_{n(1) m(2) t}^{(2)}+\sum_{\gamma=1}^{2} \sum_{p(\gamma)>m(2), n(1)} \sum_{m(2)=1}^{N(2)} \sum_{n(1)=1}^{N(1)} \Delta W_{n(1) m(2) p(\gamma) t}^{(3)}\right) .
\end{aligned}
$$

When the limiting transitions (23) and (25) are realized, the one-, two-, and three-particle potentials of discrete systems must be transformed into one-, two-, and three-particle potentials of continuous media, respectively. As a result, nonlocal models of solids and their adhesions are obtained. Within their frameworks, just as in the framework of discrete models of a solid (Born, 1954; Gusev and Lurie, 2010; Lurie and Solyaev, 2017; Kittle, 1975) and of the particle dynamics method (Hockney and Eastwood, 1981; Krivtsov, 2001), infinitesimal particles can 
have their own potential energy, interact with each other at finite distances, experiencing pair, triple, and, generally larger-order interactions.

It is assumed that the discrete systems obtained in the case of the $N$-particle partitioning of solid bodies $B$ and their supplements, corresponding to $C=\Omega \backslash B$, obey the laws of equilibrium statistical mechanics.

It is also assumed that the form of the empirical functions of the potentials of pair and triple interactions of interparticle distances is the same as in the reference, so in the current states. Therefore, the reference state is considered further, when $t=0$ and $L_{m n}=l_{m n}$.

In view of the assumed equilibrium of the reference state, homogeneity and isotropy of the materials being studied, the relationship between the potentials and distances must be invariant to the shifts and orthogonal transformations of the frame of reference. Therefore, they depend only on interparticle distances. One-particle potentials are considered equal to zero $\Delta W_{n}^{(1)}=0$.

It is held that at any value of $N$ the thermodynamic characteristics of each of the systems $\widehat{B}^{(N)}$ coincide with the thermodynamic characteristics determined in the statistical mechanics by means of a large statistical sum $\Xi$ (Huang, 1987). For its construction, it is necessary to know the masses $\Delta m^{(N)}$ of the particles $b_{n}^{(N)} \in \widehat{B}_{n}^{(N)}$, the form and parameters of the potentials of their interactions, and the equilibrium distances $l_{0}^{(N)}$ between neighboring particles. Such partitions are used for which the volumes and masses of the particles, as well as the equilibrium interparticle distances, are identical, dependent on the density of the solid material and on the number $N$ of particles into which the solid body $B$ is divided. The parameters and the form of the potentials are determined by the properties of the material under study, and by the conditions of the thermodynamics and the stability of the discrete system $\widehat{B}^{(N)}$ (Ruelle, 1999).

The large statistical sum is a functional series:

$$
\Xi^{(N)}=\sum_{n=0}^{\infty} \Xi_{n}^{(N)}=1+\sum_{n=1}^{\infty} \frac{z^{n}}{n ! h^{3 n}} \int_{\Lambda^{n}} \exp \left[-W^{(N)}\left(\vec{r}_{1}, \ldots, \vec{r}_{n}\right) / k T\right] d V_{1} \ldots d V
$$

Here

$$
\begin{aligned}
\Xi_{n}^{(N)}= & \frac{1}{n ! h^{3 n}} \int_{\Lambda^{n}} \exp \left[-\frac{H^{(N)}\left(\Delta \vec{P}_{1}^{(N)}, \ldots, \Delta \vec{P}_{n}^{(N)} ; \vec{r}_{1}, \ldots, \vec{r}_{n}\right)}{k T}\right] \\
& \times d^{3}\left(\Delta \vec{P}_{1}^{(N)}\right) \ldots d^{3}\left(\Delta \vec{P}_{n}^{(N)}\right) ; d V_{1} \ldots d V_{n}
\end{aligned}
$$

is the statistical sum constructed for $n$ particles disposed in the region $\Lambda$ and the equality $n=N$ is one of the variants (Gibbs, 1982; Kubo, 1965); $k$ and $h$ are the Boltzmann and Planck constants; $T$ is the absolute temperature; $z$ is the activity obtainable by integrating the kinetic energy of particles over all possible values and directions of their impulses; $d^{3}\left(\Delta \vec{P}_{i}^{(N)}\right)$ is the elementary volume in the space 
of impulses of the $i$ th particle; $W_{(N)}$ is the total potential energy of the interaction of all pairs and triples composed of $n$ particles. It is determined by equality (27) for $t=0$ under the condition that either all the terms containing the index $\alpha=2$ are zero, or that the bodies $B_{(1)}$ and $B_{(2)}$ are parts of one homogeneous body $B$ divided into two parts.

It is assumed that among such partitions, there exists such an $N=N^{*}$ partition, for which there corresponds the distance $l_{0}\left(N^{*}\right)$ that coincides with the average interatomic distance, whereas the dependences of the potentials of pair and triple interactions of interparticle distances coincide with the real dependences of interatomic interactions on the distances between them.

The conditions of stability of discrete systems and of their thermodynamic properties lead to the dependence of pair potentials on interparticle distances characterized by the repulsive "core" and by the "tail" tending to zero and describing the attraction (Ruelle, 1999; Ulenbek, 1971). This property ensures the convergence of the series (28).

Taking into account the above, for the discrete system $\widehat{B}^{(N)}$ corresponding to the $N$-partition of an arbitrary solid body $B$, the following dependence of the potential $\Delta W_{n m}^{(2)}$ on the interparticle distance $l_{12} \equiv l_{2} \equiv l \equiv l_{m n}$ is proposed:

$$
\begin{gathered}
\Delta W_{n m}^{(2)}=\Phi_{(N)}^{(2)}\left(l_{n m}\right) \Delta V_{m} \Delta V_{n}=\Phi_{(N)}^{(2)}(l) \Delta V_{(N)}^{2}, \\
\Phi_{(N)}^{(2)}(l)=\left\{\begin{array}{ll}
\Psi_{(N)}^{(2)} \eta_{1}(l) & 0 \leq l \leq l_{0(N)} \\
\Phi_{0}^{(2)} \eta_{2}\left[\beta^{(2)}\left(l-l_{0(N)}\right)\right] & l_{0(N)}<l<+\infty
\end{array} .\right.
\end{gathered}
$$

It is assumed that for any pair of particles $b_{m}^{(N)}$ and $b_{n}^{(N)}$ with the same distance $l_{12} \equiv l_{2} \equiv l \equiv l_{m n}$ between them (the same as between arbitrarily chosen particles $b^{(N)} \equiv b_{1}^{(N)}$ and $\left.b_{2}^{(N)}\right)$, the value of the potential $\Phi_{(N)}^{(2)}$ is the same.

In expression (30), $\beta^{(2)}, \Phi_{0}^{(2)}$, and $\Psi_{(N)}^{(2)}$ are the parameters that characterize the mechanical properties of the material determined on the basis of experimental data with account for the stability as of any of the discrete systems $\widehat{B}^{(N)}$, so of the body $B$ corresponding to each of them;

$$
l_{0(N)}=\sqrt[3]{\Delta V_{(N)}}=\sqrt[3]{V / N} .
$$

It is assumed that the functions $\eta_{1}(l)$ and $\eta_{2}(l)$ have the following properties:

$$
\begin{gathered}
\Psi_{(N)}^{(2)}>0, \quad \eta_{1}(0)=1, \quad \eta_{2}(l)<0, \quad \Phi_{0}^{(2)}>0, \\
\Psi_{(N)}^{(2)} \eta_{1}\left(l_{0(N)}\right)=\Phi_{0}^{(2)} \eta_{2}\left(l_{0(N)},\left.\quad \frac{d \eta_{1}}{d l}\right|_{l=l_{0}}=\left.\frac{d \eta_{2}}{d l}\right|_{l=l_{0(N)}}=0,\right.
\end{gathered}
$$




$$
\left.\Psi_{(N)}^{(2)} \frac{d^{2} \eta_{1}}{d l^{2}}\right|_{l=l_{0(N)}}=\left.\Phi_{0}^{(2)} \frac{d^{2} \eta_{2}}{d l^{2}}\right|_{l=l_{0(N)}}>0 .
$$

These properties do not contradict to the properties of the pair potentials of stable discrete systems presented in Hockney and Eastwood (1981).

There are a number of ways of describing triple potential interactions [e.g., (Barash, 1988; Tersoff, 1988; Erkoc, 1997; Poluektov and Soroka, 2015)]. It is seen from these works that the use is made of the functions that do not have a "repulsive" part. This is taken into account in what follows.

The three-particle potential is selected in the form that accounts for the fact [following the pairs in the Mayer scheme (Mayer and Goeppert-Mayer, 1980)] that one particle interacts simultaneously with two other particles that do not interact with each other (Poluektov and Soroka, 2015):

$$
\begin{gathered}
\Delta W_{m n p}^{(3)}=\Phi_{(N)}^{(3)}\left(l_{m n} l_{m p}\right) \Delta V_{m} \Delta V_{n} \Delta V_{p}=\Phi_{(N)}^{(3)}\left(l_{1} l_{2}\right) \Delta V_{(N)}^{(3)}, \\
l_{m n} \equiv l_{j}, j=2,3, \\
\Phi_{(N)}^{(3)}\left(l_{2}, l_{3}\right)=\Phi_{0}^{(3)} \eta_{2}\left(\beta^{(3)} l_{2}\right) \eta_{2}\left(\beta^{(3)} l_{3}\right), 0<l_{j}<\infty .
\end{gathered}
$$

Taking into account the condition that the interparticle potentials coincide at $N=N^{*}$ with real interatomic potentials, it is assumed that the rates of decrease in the potentials of the pair $\left(l>l_{0}\left(N^{*}\right)\right)$ and triple $(l>0)$ interactions, characterized by the parameters $\beta^{(2)}$ and $\beta^{(3)}$ are equal to or the real velocities differ from them slightly. That is, for all the values of $N$, the parameters $\beta^{(2)}$ and $\beta^{(3)}$ are the same.

At any value of the number $N$ and, consequently, at any value of the distance $l_{o(N)}$, the following conditions are fulfilled:

$$
\begin{gathered}
\forall N, \quad \forall l_{0(N)} ; \quad 0<\int_{\Lambda_{0(N)}} \Phi_{(N)}^{(2)}(l) d V=4 \pi \Psi_{(N)}^{(2)} \int_{0}^{l_{0}^{(N)}} l^{2} \eta_{1}(l) d l=I_{0}^{(2)}=\text { const }<+\infty \\
\forall N, \quad \forall l_{0(N)}: \frac{d}{d l_{0}} \int_{\Lambda \backslash \Lambda_{0}} \Phi_{(N)}^{(2)}(l) d V=0 \\
\forall N, \quad \forall l_{0(N)} ; \int_{\Lambda_{(N)}} \Phi_{(N)}^{(2)}\left(l_{12}\right) d V_{2}>0
\end{gathered}
$$


Here $\Lambda_{0}$ is the region bounded by a sphere of radius $l=l_{0}$, in the center of which there is one of the two interacting particles. The second integral in condition (36) is written in a spherical coordinate system $(l, \theta, \varphi)$ with the origin $l=0$ at the center of this sphere.

When $N \rightarrow \infty$, the upper limit $l_{0(N)}=\sqrt[3]{V / N} \rightarrow 0$, the region $\Lambda_{0}$ is shrunk to a point, i.e., to the center of inertia of one of the pair of interacting particles $\Delta B$ and $\Delta B \rightarrow d B$, the potential $\Phi_{(N)}^{(2)}(l) \Delta V_{(N)}^{2}$ of the discrete system tends to the interaction potential

$$
\Phi_{\infty}^{(2)}(l) d V d V_{2} \equiv \Phi^{(2)}(l) d V d V_{2}
$$

of infinitesimal particles of a continuous medium. The dependence $\Phi_{(N)}^{(2)}(l)$ becomes similar to the Dirac delta function. In this case, when $N \rightarrow \infty$ and $l_{0}(N) \rightarrow 0$,

$$
\Phi_{(N)}^{(3)}\left(l_{2}, l_{3}\right)=\Phi_{(\infty)}^{(3)}\left(l_{2}, l_{3}\right) \equiv \Phi^{(3}\left(l_{2}, l_{3}\right)
$$

Condition (36) is a consequence of the thermodynamic condition of the discrete system of interacting material points (Ruelle, 1999). Condition (37) makes it possible to maintain thermodynamic properties for the system of particles $B \Delta \rightarrow d B$ that form the continuous medium $\Omega$ and its component part $B$. Inequality (38) is a condition for the thermodynamic stability of the system in the absence of triple interactions.

In fact, if we integrate Eq. (38) with respect to the region $\Lambda_{(N)}$, we can obtain the condition imposed [as shown in Ruelle (1999)] for the sum of all pair interactions in a stable system $\widehat{B}^{(N)}$ :

$$
W=W_{(N)}\left(\vec{r}_{1}, \ldots, \vec{r}_{N}\right)=\sum_{i<j}^{N} \Delta W_{i j}>0 .
$$

In this case, starting with some number $n_{0}$, for all subsequent $n>n_{0}$ the following inequality holds:

$$
W_{(N)}\left(\vec{r}_{1}, \ldots, \vec{r}_{n}\right)=\sum_{i<j}^{n} \Delta W_{i j}>0
$$

Then the general term of the series (28) satisfies the inequality

$$
u_{n}=\frac{z^{n}}{n ! h^{3 n}} \int_{\Lambda^{n}} \exp \left[-W_{(N)}\left(\vec{r}_{1}, \ldots, \vec{r}_{n}\right) / k T\right]<\frac{1}{n ! h^{3 n}}\left(\frac{z V}{h^{3}}\right)^{n} .
$$

The series (28) itself converges in this case. 
Conditions (38) and (41) are satisfied under the assumption that

$$
\int_{\Lambda_{0}} \Phi_{(N)}^{(2)} d V^{2}=\xi\left|\int_{\Lambda_{\infty} \backslash \Lambda_{0}} \Phi_{(N)}^{(2)} d V_{2}\right|, \xi>1
$$

For each value of $N$, this condition makes it possible to express the parameter $\Psi_{(N)}^{(2)}$ in terms of the parameters $\beta^{(2)}, \Phi_{0}^{(2)}$, and $l_{0(N)}$.

It is suggested that the influence of triple interactions on the stability of a discrete system be considered as follows.

For the reference state, on the basis of Eq. (2), we can write the expression for determining the potential energy of interaction of particles in a continuous medium:

$$
W=\int_{\Lambda} w(\vec{R}) d V=\int_{\Lambda}\left\{\int_{\Lambda_{t}} \Phi^{(2)}\left(L_{12}\right) d V_{t 2}+\int_{\Lambda} d W_{t 2} \int_{\Lambda_{t}} \Phi^{(3)}\left(L_{12}, L_{13}\right) d V_{t 3}\right\} d V .
$$

Here the expression in the curly brackets can be regarded as a generalized potential of pair interactions. For a discrete analogue, the conclusions made in Ruelle (1999) on the stability of discrete systems with a positive sum of pair potentials of their particles are applicable. The integral of the first term is the potential energy of all pair interactions of the solid body $B$, which, due to Eq. (38), is positive. The sign of the second term depends on the sign of the parameter $\Phi_{0}^{(3)}$. In the case of it being negative, the entire sum and the values $W=W_{(N)}$ may turn out to be negative for both the solid body and the discrete system. The system will turn out to be unstable.

Relations (29)-(39) allow us to establish the dependence of the potentials of the interaction of particles of a continuous medium on the distances between them. It is suggested to express their parameters $(\vartheta, \ldots, \theta) \equiv\left(\beta^{(2)}, \beta^{(3)}, \Phi_{0}^{(3)}, \Phi_{0}^{(3)}\right)$ in terms of the elastic state characteristics of the material corresponding to the body $B$ of the infinite medium $\Omega$.

It is believed that the dependences $\Phi^{(2)}\left(L_{2}\right)$ and $\Phi^{(3)}\left(L_{2}, L_{3}\right)$ can be represented by second-order polynomials relative to $\Delta \vec{u}_{j}$. In this case, the vectors $\Delta \vec{u}_{j}$ are represented as the sums of the first two terms of the series in terms of the external powers of the vectors $\vec{l}_{j}$ (Shorkin, 2011).

As a result, expression (10) for the bulk density of the work of internal forces on these deformations takes the following form:

$$
w=w-P_{0}^{(1)} \cdot \cdot(\nabla \vec{u})^{T}=(\nabla \vec{u}) \cdot C^{(1,1)} \cdot \cdot(\nabla \vec{u})^{T}+\left(\nabla^{2} \vec{u}\right) \cdot C^{(2,2)} \cdot \cdot\left(\nabla^{2} \vec{u}\right)^{T} .
$$

Here

$$
P_{0}^{(1)}=\int_{\Lambda(\Omega)}\left(\frac{d \Phi^{(2)}}{d \vec{l}}\right) \vec{l} d V_{2}+\sum_{k=2}^{3} \int_{V}\left[\int_{V}\left(\frac{d \Phi^{(3)}}{d \vec{l}_{k}}\right) \vec{l}_{k} d V_{2}\right] d V_{3}
$$


is the tensor characterizing the initial stressed state, caused by interparticle potential interactions in the reference state;

$$
C^{(m, m)}=\int_{\Lambda(\Omega)} \vec{l}\left(\frac{d^{2} \Phi^{(2)}}{d \vec{l}^{2}}\right) \vec{l} d V_{2}+\sum_{k, q=2}^{3} \int_{\Lambda(\Omega)}\left[\int_{\Lambda(\Omega)} \vec{l}_{k}\left(\frac{d^{2} \Phi^{(3)}}{d \vec{l}_{k} d \vec{l}_{q}}\right) \vec{l}_{q} d V_{2}\right] d V_{3}, \quad(m=1,2)
$$

are the tensors characterizing the elastic properties of the material of the medium $\Omega$.

When the investigated body $B$ occupies a limited region $\Lambda(B) \equiv \Lambda$, tensors (46) and (47) are variables depending on the distance of their determination point to the boundary of the region $\Lambda(B) \equiv \Lambda$. In this case, the initial stress tensor $P_{0}^{(2)}$ performing work on the generalized displacement $\left(\nabla^{2} \vec{u}\right)$ turns to be not equal to zero as well as the tensors $C^{(1,2)}$ and $C^{(2,1)}$ characterizing the ability of the material to realize cross effects. With increasing distance from the boundary to the point of determination of these tensors, they tend to zero.

The symmetry properties satisfied by definitions (47) indicate that the right-hand side of expression (46) corresponds to the gradient theory of Jaramillo (Belov and Lurie, 2014; Gusev and Lurie, 2017). In this case, the tensors $C^{(1,1)}$ and $C^{(2,2)}$ coincide structurally with the Jaramillo tensors $C^{D(1,1)}$ and $C^{D(2,2)}$, with a linear dependence existing between the two nonclassical tensors.

The right-hand side of expression (45) is a quadratic form with respect to the gradients of deformation. For a stable system, such as the medium $\Omega$, it is positively definite. The material constants of the tensors $C^{D(1,1)}$ and $C^{D(2,2)}$ satisfy the conditions for their positive definiteness. Therefore, assuming that $C^{(1,1)}=C^{D(1,1)}$ and $C^{(2,2)}=C^{D(2,2)}$, we may obtain conditions for determining the parameters $\beta^{(1)}$, $\beta^{(3)}, \Phi_{0}^{(2)}$, and $\Phi_{0}^{(3)}$. To ensure uniqueness, it is permissible that $\beta^{(2)}=\beta^{(3)}=\beta$. The parameters $\beta, \Phi_{0}^{(2)}$, and $\Phi_{0}^{(3)}$ found in this way correspond to the requirement of the stability of a continuous elastic medium. It is suggested to determine them experimentally on the basis of two classical tensile and shear experiments, with the third one being an experiment on constructing a nonlinear dispersion law on the propagation of longitudinal high-frequency acoustic waves (Kittle, 1975). A description of the nonlinearity of the dispersion law for transverse waves can serve as a verification of the results obtained.

It is proposed to use the following dependence as one of the possible variants of the function $\eta_{2}\left[\beta\left(l-l_{0(N)}\right)\right]$ entering into expression (30):

$$
\eta_{2}=\exp \left(-2 \beta\left(l-l_{0(N)}\right)\right)-2 \exp \left(-\beta\left(l-l_{0(N)}\right)\right), \quad l>l_{0(N)}
$$

The requirement (36)-(38) imposed on the function $\Psi_{(N)}^{(2)} \eta_{1}(l)$, makes it possible not to use its concrete form in the calculations carried out. It is assumed in equality (43) that $\xi=2$. As a result, the following expressions (Shorkin, 2011) are obtained for the parameters $\beta, \Phi_{0}^{((2)}$, and $\Phi_{0}^{((3)}$ : 


$$
\begin{aligned}
\left(\frac{\Phi_{0}^{(2)}}{\beta^{3}}\right) & =\frac{4 \mu+45 \lambda}{294 \pi}=\frac{E}{294 \pi} \frac{2+41 v}{(1+v)(1-2 v)}, \\
\left(\frac{\Phi_{0}^{(3)}}{\beta^{6}}\right) & =\frac{27(\lambda-\mu)}{1764 \pi^{2}}=\frac{27 E}{1764 \pi^{2}} \frac{4 v-1}{2(1+v)(1-2 v)}, \\
\beta & =\frac{1}{2} \sqrt{3 \pi\left(\frac{f_{0}}{f_{1}}\right)^{15\left(\frac{\Phi_{0}^{(2)}}{\beta^{3}}\right)+\left(\frac{1563 \pi}{4}\right)\left(\frac{\Phi_{0}^{(3)}}{\beta^{6}}\right)}} .
\end{aligned}
$$

In expression (51), $f_{0}$ and $f_{1}$ are experimentally determined parameters characterizing the approximation of the dispersion law by the function $\omega^{2}=f\left(K^{2}\right)=$ $f_{0} K^{2}-f_{1} K^{4}$, in which $\omega$ and $K$ are the circular frequency and the wave number, respectively. Only the parameter $f_{1}$ is new with respect to $\lambda$ and $\mu$, since $f_{0}$ is expressed in terms of the classical characteristics of linear elastic state (Kittle, 1975).

In the case of using the theoretical dispersion law (Kittle, 1975),

$$
\beta=\frac{3 \sqrt{\pi}}{l_{a t}} \sqrt{\frac{15\left(\frac{\Phi_{0}^{(2)}}{\beta^{3}}\right)+\left(\frac{1563 \pi}{4}\right)\left(\frac{\Phi_{0}^{(3)}}{\beta^{6}}\right)}{2 \mu+\lambda} .}
$$

Here $l_{a t}=\frac{1}{\sqrt[3]{n}}$, where $n$ is the number of atoms per unit volume.

\section{ADHESION OF ELASTIC MATERIALS}

It is proposed to calculate the strength of the adhesion of two bodies $B_{(\alpha)}$ and $B_{(\beta)}(\alpha, \beta=1,2 ; \alpha \neq \beta)$ on the basis of expression (18) with account for expression (11) constructed for the moment $t=0$. It is seen that in order to perform the calculations it is necessary to know the dependences of the potentials of the pair and triple interactions of particles consisting of different materials on the distances between them. Information on this is obtained as follows.

It is assumed that the interaction of particles $d B_{(\alpha)}$ and $d B_{(\beta)}$ belonging to bodies $B_{(\alpha)}$ and $B_{(\beta)}$ made of different materials is identical to the interaction of the same particles when they form the particle $d B_{(s s)}$ of the two-component solid solution $B_{(s s)}$ of these materials with the corresponding volume concentrations $c_{(\alpha)}$ and $c_{(\beta)}$ $\left(c_{(\alpha)}+c_{(\beta)}=1\right)$ (Dolgov et al., 2015).

To determine the parameters of the required dependences: $\beta_{(12)}, \Phi_{0(12)}^{(2)}, \Phi_{0(112)}^{(3)}$, and $\Phi_{0(122)}^{(3)}$ it is proposed to use the dependences of Young's modulus and of the shift modulus of the two-component mixture $B_{(s s)}$ on the concentration of its components $B_{(\alpha)}$ and $B_{(\beta)}$. The particles $d B_{(\alpha)}$ and $d B_{(\beta)}$ have the same displacement 
vectors and their gradients, experience a pair and a triple interaction between themselves. It is realized with the help of nonlocal potential forces, as in a homogeneous medium that has the characteristics $\beta_{(s s)}$, $\Phi_{0(s s)}^{(2)}$, and $\Phi_{0(s s)}^{(3)}$ for a nonlocal description, and $\lambda_{(s s)}$ and $\mu_{(s s)}$ for a local one. The potentials of the interparticle interactions of the material $B_{(s s)}$ are subjected to the same requirements as the materials of really homogeneous bodies $B_{(\alpha)}$ and $B_{(\beta)}$. Consequently, Eqs. (49)-(52) can be used for communication of the group of parameters $\left(\beta_{(s s)}, \Phi_{0(s s)}^{(2)}\right.$, and $\left.\Phi_{0(s s)}^{(3)}\right)$ with the group $\left(f_{0(s s)}, \lambda_{(s s)}\right.$, and $\left.\mu_{(s s)}\right)$ [provided that the law (48) is valid]. The concentration dependences of the parameters $f_{0(s s)}, \lambda_{(s s)}$, and $\mu_{(s s)}$ are determined experimentally. They can also be determined theoretically by means of the methods presented in Lurie et al. (2016a,b) and Lurie and Solyaev (2010).

In this paper, a simplified version of the concentration dependences of the Young's $E_{(s s)}$ and the shift $G_{(s s)}$ moduli is selected, which corresponds to the Reuss model, is chosen, without taking into account the "exchange" fraction of the mixture generated by the adhesive interaction of its elements:

$$
\frac{1}{E_{(s s)}}=\frac{c_{(\alpha)}}{E_{(\alpha)}}+\frac{c_{(\beta)}}{E_{(\beta)}}, \frac{1}{G_{(s s)}}=\frac{c_{(\alpha)}}{G_{(\alpha)}}+\frac{c_{(\beta)}}{G_{(\beta)}} .
$$

The Poisson ratio $v_{(s s)}$ and Lame parameters $\lambda_{(s s)}$ and $v_{(s s)}$ are expressed in terms of the Young's $E_{(s s)}$ and the shift $G_{(s s)}$ moduli by the formulas well known in the linear theory of elasticity (Nowacki, 1975).

The potential energy of interaction of two infinitesimally close particles $d B_{(s s) j}$ $(j=1,2)$ that have the volumes

$$
d V_{(s s) j}=d V_{(\alpha) j}+d V_{(\beta) j}=(1-c) d V_{(s s) j}+c d V_{(s s) j},
$$

where $c=c_{(\beta)}$ and $1-c=c_{(\alpha)}$, is equal, on the one hand, to $\Phi_{(s s) 0}^{(2)} d V_{(s s) 1} d V_{(s s) 2}$ and, on the other hand (taking into account that $d B_{(s s) j}=d B_{(\alpha) j} \bigcup d B_{(\beta) j}$ ), to

$$
\left[(1-c)^{2} \Phi_{0(11)}^{(2)}+2 c(1-c) \Phi_{0(12)}^{(2)}+c^{2} \Phi_{0(22)}^{(2)}\right] d V_{(s s) 1} d V_{(s s) 2} \text {. }
$$

Therefore, the following equality holds:

$$
\Phi_{(s s) 0}^{(2)}=(1-c)^{2} \Phi_{(11) 0}^{(2)}+2 c(1-c) \Phi_{(12) 0}^{3)}+c^{2} \Phi_{(22) 0}^{(2)} .
$$

Considering the interaction of three infinitesimally close particles $d B_{(s s)} p(p=1,2,3)$, we can obtain the expression

$$
\begin{aligned}
\Phi_{(s s) 0}^{(3)} & =(1-c)^{3} \Phi_{(111) 0}^{(3)}+3 c(1-c)^{2} \Phi_{(112) 0}^{(3)} \\
& +3 c^{2}(1-c) \Phi_{(122) 0}^{(3)}+c^{3} \Phi_{(222) 0}^{(3)} .
\end{aligned}
$$

Volume 9, Issue 4, 2018 
The calculation of the volumetric density of the potential energy of all pair interactions of an arbitrary particle $d B_{(s s)} \equiv d B_{(s s)} 1$ with its environment inside $\Omega_{(s s)}$ leads to the equality

$$
\left(\frac{\Phi_{(s s) 0}^{(2)}}{\beta_{(s s)}^{3}}\right)=(1-c)^{2}\left(\frac{\Phi_{(11) 0}^{(2)}}{\beta_{(11)}^{3}}\right)+2 c(1-c)\left(\frac{\Phi_{(12) 0}^{(2)}}{\beta_{(12)}^{3}}\right)+c^{2}\left(\frac{\Phi_{(22) 0}^{(2)}}{\beta_{(22)}^{3}}\right) .
$$

The volume density of the potential energy of all triple interactions of the same particle $d B_{(s s)} \equiv d B_{(s s)} 1$ with its environment inside $\Omega_{(s s)}$ is given by

$$
\begin{aligned}
\left(\frac{\Phi_{(s s) 0}^{(3)}}{\beta_{(s s)}^{6}}\right) & =(1-c)^{3}\left(\frac{\Phi_{(111) 0}^{(3)}}{\beta_{(11)}^{6}}\right)+3 c(1-c)^{2}\left(\frac{\Phi_{(112) 0}^{(3)}}{\beta_{(11)}^{3} \beta_{(12)}^{3}}\right) \\
& +3 c^{2}(1-c)\left(\frac{\Phi_{(122) 0}^{(3)}}{\beta_{(22)}^{3} \beta_{(12)}^{3}}\right)+c^{3}\left(\frac{\Phi_{(222) 0}^{(3)}}{\beta_{(22)}^{6}}\right) .
\end{aligned}
$$

In expressions (54)-(57), the parameters $\Phi_{(11) 0}^{(2)}, \Phi_{(22) 0}^{(2)}, \Phi_{(111) 0}^{(3)}, \Phi_{(22) 0}^{(3)}, \beta_{(11)}$, and $\beta_{(22)}$ are known. They are determined for each of the contacting homogeneous materials by Eqs. (49)-(52). The concentration dependencies of the left-hand sides of expressions (54) and (56), which are found on the basis of the same equations, are approximated by the second-degree polynomials, and for expressions (55) and (57), third-degree ones with respect to the argument $c$. Their equating with the polynomials in the right-hand sides of the same equations makes it possible to construct expressions for calculating the parameters with $\beta_{(12)}, \Phi_{(12) 0}^{(2)}, \Phi_{(112) 0}^{(32)}$, and $\Phi_{(122) 0}^{(3)}$.

Using the presented mathematical apparatus, having information about the characteristics of the elastic properties of the materials of two bodies under the assumption of their homogeneity, isotropy, and linear elasticity, we can calculate the strength of their adhesion interaction. In particular, it is suggested to solve the probe-sample interaction problem, experimentally investigated in Dedkov et al. (2009), by a method based on the results of the works of Derjaguin (1934a,b) and Goryacheva and Makhovskaya (2001).

A semi-infinite body, i.e., a body $B_{(1)}$ bounded by the plane $A_{(1)}$, was considered as a sample. The surface $A_{(2)}$ of the probe-body $B_{(12)}$ has a spherical shape with a known radius $R$. The problem has a cylindrical symmetry. The vector $\vec{h}$ in expressions (15)-(18) is directed along the axis $O z$. Therefore, a cylindrical coordinate system $O \varphi, r, z$ is used with an origin at the point $O$, which is located on the plane $A_{(1)}$. The axis $O z$ is perpendicular to $A_{(1)}$, directed towards the body $B_{(2)}$. The center of the sphere is at a distance $d$ from the plane $A_{(1)}$. As a result, the point of the sphere nearest to the plane $A_{(1)}$ is the point $M: \varphi$ is any, $r=0$, $z=z_{M} \equiv h=z_{0}=d-R$.

Further, the spherical surface is replaced by a stepped one as follows: 
1. The segment $\left[z_{M}=z_{0} ; d\right]$ is divided by points $z_{i}(i=0,1, \ldots, n-1)$ into segments of length $\Delta z_{i}=z_{i+1}-z_{i}=\eta$. So that, $z_{i+1}=z_{i}+(i+1) \eta, n=\left(z_{n}-z_{0}\right) / \eta$.

2. The values of radii $r_{i}$ of the sections of the sphere by planes $z=z_{i}$ are calculated by the formulas defining them: $r_{i+1}^{2}=R^{2}-[R-(i \eta)]^{2}$, where $i=1, \ldots, n-1$.

3. The spherical surface is replaced by a stepped one formed by flat rings $0<\varphi \leq 2 \pi, z=z_{i}, r_{i-1}<r<r_{i}$.

4. The problem of the interaction of semi-infinite bodies is solved. They consist of materials $B_{(1)}$ and $B_{(2)}$ limited to parallel planes. The distance between the planes is $z$. The solution determines the values $p_{i}=p\left(z_{i}\right)$ of the force that acts on the part of the body $B_{(1)}$ per unit area of the body $B_{(2)}$.

It is taken into account that the adhesion force is a volumetric one. Therefore, the replacement of a spherical surface in an elementary section by a plane, perpendicular to $\mathrm{Oz}$ does not influence the result of calculations. The basis is the solution of the problem of the interaction of two semi-infinite bodies (Dolgov et al., 2015).

5. For the action force $F_{(12)}$ from the side of the body $B_{(1)}$ on the body $B_{(2)}$, an expression is constructed that determines it:

$$
F_{(12)}=\pi\left[p_{0} r_{0}^{2}+p_{1}\left(r_{1}^{2}-r_{0}^{2}\right)+\ldots+p_{n-1}\left(r_{n-1}^{2}-r_{0}^{2}\right)\right] .
$$

The process is repeated the needed number of times for the given calculation accuracy.

\section{COMPARISON OF CALCULATIONS WITH EXPERIMENTAL DATA}

Figure 1 shows the values of the Van der Waals force of interaction between the probe and the sample, which is determined for different distances between them

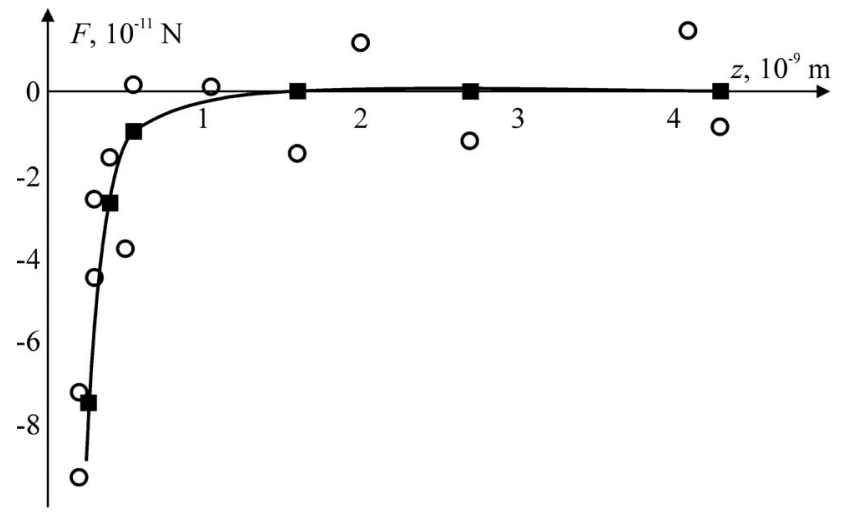

FIG. 1: The experimental (०) (Dedkov et al., 2009) and theoretical (घ) dependences of the Van der Waals force of interaction between the probe and sample, which are made of platinum and carbon, on the distances between them

Volume 9, Issue 4, 2018 
using the experiment (Dedkov et al., 2009), and by calculation. Comparing them among themselves, we can see that they are in satisfactory agreement.

\section{CONCLUSIONS}

The paper presents a method of theoretical calculation of the adhesion forces of a probe and a sample in an atomic-force microscope, depending on the distance between them. The method is based on a specially constructed nonlocal model of the adhesion interaction of solids. It is based on taking into account the pair and triple potential interactions of infinitesimal particles of a continuous elastic medium that can be located at finite distances from each other. The model is constructed by analogy with the model of discretely located potentially interacting particles possessing finite masses, impulses, and energies.

The potentials of interaction of particles of a continuous medium are obtained on the basis of the potentials of the interaction of particles of a discrete system. The stability of the system of particles of a continuous medium is ensured from both the point of view of statistical mechanics (from the condition of convergence of the series defining a large statistical sum) and the point of view of the mechanics of continuous media (from the condition of fulfilling the Born stability criterion). To determine the parameters of the potentials interaction, it is sufficient to know the Young's modulus and Poisson's coefficients of the materials under study. This makes the model convenient for carrying out practical calculations, in particular, on the evaluation of the dependence of the interaction force between the probe and the sample on the distance between them in an atomic-force microscope. The adequacy of their reality results and satisfactory accuracy is confirmed by comparison with known data.

\section{ACKNOWLEDGMENT}

The work was carried out within the framework of the basic part of the State task for 2017-2019 years, project code 1.5265.2017/BCH.

\section{REFERENCES}

Barash, Yu.S., Van der Waals Forces, Moscow: Nauka Press, 1988 (in Russian).

Belov, P.A. and Lurie, S.A., Mathematical Theory of Defective Media. Gradient Theories of Elasticity. Formulations. Hierarchy. Comparative Analysis. Applications, Palmarium Academic Publishing, 2014 (in Russian). ISBN 978-3-639-73761-5

Berinskii, I.E., Ivanova, E.A., Krivtsov, A.M., and Morozov, N.F., Application of Moment Interactions for Construction of Stable Model of the Graphite Crystal Lattice, Mech. Solids, vol. 42, no 5, pp. 663-671, 2007. DOI: 10.3103/S0025654407050020

Born, M. and Huang, H., Dynamical Theory of Crystal Lattices, Oxford: Clarendon Press, 1954. DOI: $10.1119 / 1.1934059$

Born, M. and Oppenheimer, R., Zur Quantentheorie der Molekeln, Annalen der Physik, no. 20, pp. 457-484, 1927. 
Byzov, A.P. and Ivanova, E.A., Mathematical Modeling of Momentum Interactions of Particles with Rotational Degrees of Freedom, Nauch.-Tekh. Vedomosti SPbGPU, no. 2, pp. 260-268, 2007 (in Russian).

Cappella, B. and Dietler, G., Force-Distance Curves by Atomic-Force Microscopy, Surface Sci. Rep., vol. 34, pp. 1-104, 1999. DOI: 10.1016/S0167-5729(99)00003-5

Dedkov, G.V., Kanametov, A.A., and Dedkova, E.G., Electrostatic and van der Waals Forces in the Air Contact between the Atomic-Force Microscope Probe and a Conducting Surface, Zh. Tekh. Fiz., vol. 79, no. 12, pp. 79-85, 2009. DOI: 10.1134/S1063784209120147

Derjaguin, B., Molecular Theory of Outer Friction, Z. Physik, vol. 88, nos. 9-10, pp. 661-675, 1934a.

Derjaguin, B., Untersuchungen uber die Reibung und Adhasion, IV. Theorie des Anhaftens kleiner Teilchen, Kolloid Z., vol. 69, pp. 155-164, 1934b.

Derjaguin, B.V., Muller, V.M., and Toporov, Y.P., Effect of Contact Deformations on the Adhesion of Particles, J. Colloid Interface Sci., vol. 53, no. 2, pp. 314-326, 1975. DOI: 10.1016/00219797(75)90018-1

Dolgov, N.A., Romashin, S.N., Frolenkova, L.Yu., and Shorkin, V.S., A Model of Contact of Elastic Bodies with Account for Their Adhesion, Nanosci. Technol.: An Int. J., vol. 6, no 2, pp. 117-133, 2015. DOI: 10.1615/NanomechanicsSciTechnolIntJ.v6.i2.30

Eringen, C.A., Nonlocal Continuum Field Theories, New York: Springer-Verlag, 2002.

Erkoc, S., Empirical Many-Body Potential Energy Function Used in Computer Simulation of Condensed Matter Properties, Phys. Rep., vol. 278, no 2, pp. 80-105, 1997.

Frolenkova, L.Yu. and Shorkin, V.S., Method of Calculating the Surface and Adhesion Energies of Elastic Bodies, PNRPU Mech. Bull., no. 1, pp. 235-259, 2013.

Frolenkova, L.Yu. and Shorkin, V.S., Surface Energy and Adhesion Energy of Elastic Bodies, Mech. Solids, vol. 52, no 1, pp. 62-74, 2017. DOI: 10.3103/S0025654417010083

Gibbs, J.W., Thermodynamics. Statistical Mechanics, Moscow: Nauka Press, 1982 (Russian translation).

Goryacheva, I.G. and Makhovskaya, Yu.Yu., Adhesive Interaction of Elastic Bodies, J. Appl. Math. Mech., vol. 65, no. 2, pp. 273-282, 2001. DOI: 10.1016/S0021-8928(01)00031-4

Grekova, E.F., Momentum Interactions of Solid Bodies, Proc. 23rd School on Analysis and Synthesis of Nonlinear Mechanical Oscillating Systems, St.Peterburg: Izd. IPMashRAN, pp. 218-228, 1996 (in Russian).

Gusev, A.A. and Lurie, S.A., Strain-Gradient Elasticity for Bridging Continuum and Atomistic Estimates of Stiffness of Binary Lennard-Jones Crystals, Adv. Eng. Mater., vol. 12, no. 6, pp. 529-533, 2010. DOI: $10.1002 /$ adem.201000004

Gusev, A.A. and Lurie, S.A., Symmetry Conditions in Strain Gradient Elasticity, Math. Mech. Solids, vol. 22, no. 4, pp. 683-691, 2017. DOI: 10.1177/1081286515606960

Hamaker, H.C., The London-Van der Vaals Attraction between Spherical Particles, Physica, vol. 4, no 10, pp. 1059-1072, 1937.

Hockney, P.W. and Eastwood, J.W., Computer Simulation Using Particles, Boca Raton, FL: CRC Press, 1981. DOI: 10.1201/9781439822050

Huang, K., Statistical Mechanics, 2nd Ed., New York: John Wiley and Sons, Inc., 1987.

Ivanova, E.A., Krivtsov, A.M., and Morozov, N.F., Macroscopic Relations of Elasticity for Complex Crystal Latices Using Moment Interaction at Microscale, Appl. Math. Mech., vol. 71, no. 4, pp. 543-561, 2007.

Volume 9, Issue 4, 2018 
Jonson, K.K., Kendall, K.L., and Roberts, A.D., Surface Energy and the Contact of Elastic Solids, Proc. Roy. Soc. London, Series A, Math. Phys. Sci., vol. 324, no. 1558, pp. 301-313, 1971. DOI: $10.1098 /$ rspa.1971.0141

Kittle, C., Introduction to Solid State Physics, New York: Wiley and Sons, 1975.

Krivtsov, A.M., Second-Order Equation of State for Lennard-Jones Chain, Proc. XXVIII Summer School on Nonlinear Oscillations in Mechanical Systems. Actual Problems in Mechanics, St. Petersburg, Russia, vol. 1, pp. 79-90, 2001.

Kubo, P., Statistical Mechanics, North-Holland, Amsterdam: Elsevier Science Publishers, 1965.

Lifshitz, E.M., Theory of Molecular Attractive Forces between Solids, Zh. Eksp. Teor. Fiz., vol. 2, pp. 73-83, 1956.

Lurie, S., Volkov-Bogorodskii, D., and Tuchkova, N., Exact Solution of Eshelby-Christensen Problem in Gradient Elasticity for Composites with Spherical Inclusions, Acta Mechanica, no. 227, pp. 127-138, 2016a. DOI: 10.1007/s00707-015-1422-3

Lurie, S., Volkov-Bogorodsky, D., Solyaev, Y., Rizahanov, R., and Agureev, L., Multiscale Modeling of Aluminum-Based Metal-Matrix Composites with Oxide Nanoinclusions, Comput. Mater. Sci., vol. 116, pp. 62-73, 2016b. DOI: 10.1016/j.commatsci.2015.12.034

Lurie, S.A. and Solyaev, Y.O., Identification of Gradient Elasticity Parameters based on Interatomic Interaction Potentials Accounting for Modified Lorentz-Berthelot Rules, Phys. Mesomech., vol. 20, no. 4, pp. 392-398, 2017. DOI: 10.1134/S1029959917040038

Lurie, S.A. and Solyaev, Yu.O., Modified Eshelby Method in the Problem of Determining Effective Properties with Micro- and Nanoinclusions, Vestn. PGTU, Ser. Mekhanika, issue Mathematical Modeling of Physical and Mechanical Processes, no. 1, pp. 80-90, 2010 (in Russian).

Lurie, S.A., Belov, P.A., and Rabinskiy, L.N., Model of Media with Conserved Dislocation. Special Cases: Cosserat Model, Aero-Kuvshinskii Media Model, Porous Media Model, in F. dell'Isola, V. Eremeyev, and A. Porubov (Eds.), Advances in Mechanics of Microstructured Media and Structures. Advanced Structured Materials, vol. 87, pp. 215-249, New York: Springer, 2018. DOI: 10.1007/978-3-319-73694-5_13

Mayer, E.J. and Goeppert-Mayer, M., Statistical Mechanics, Moscow: Mir Press, 1980 (Russian translation).

Maugis, D., Adhesion of Spheres: The JKR-DMT Transition Using a Dugdale Model, J. Colloid Interface Sci., vol. 150, no. 1, pp. 243-269, 1992. DOI: 10.1016/0021-9797(92)90285-T

Maugis, D., Contact, Adhesion and Rupture of Elastic Solids, Berlin Heidelberg/New York: SpringerVerlag, 2000.

Nowacki, W., Theory of Elasticity, Moscow: Mir Press, 1975 (Russian translation).

Palmov, V.A., Fundamental Laws of Nature in Nonlinear Thermomechanics of Deformable Bodies: Tutorial, St. Petersburg: Izd. SPbGPU, 2008 (in Russian).

Poluektov, Yu.M. and Soroka, A.A., The Equation of State and the Quasiparticle Mass in the Degenerate Fermi System with an Effective Interaction, East Eur. J. Phys., vol. 2, no. 3, pp. 40-49, 2015.

Romashin, S.N., Presnetsova, V.Yu., Frolenkova, L.Yu., and Shorkin, V.S., Method for Calculating the Characteristics of Elastic State Media with Internal Degrees of Freedom, Adv. Struct. Mater., vol. 42, no. 1, pp. 363-376, 2016. DOI: 10.1007/978-3-319-31721-2_16

Ruelle, D., Statistical Mechanics: Rigorous Results, France: Institut des Hautes Etudes Scientifiques, 1999.

Shorkin, V.S., Nonlinear Dispersion Properties of High-Frequency Waves in the Gradient Theory of Elasticity, Mech. Solids, vol. 6, pp. 898-912, 2011. DOI: 10.3103/S0025654411060094 
Tersoff, J., New Empirical Approach for the Structure and Energy of Covalent System, Phys. Rev., vol. 37, no. 12, pp. 6991-6999, 1988. DOI: 10.1103/PhysRevB.37.6991

Truesdell, K., A First Course in Rational Continuum Mechanics, Moscow: Nauka Press, 1975 (Russian translation).

Ulenbek, G., Fundamental Problems of Statistical Mechanics, Usp. Fiz. Nauk, vol. 103, no. 2, pp. 275-318, 1971.

Zhilin, P.A., Rational Mechanics of Continua: Tutorial, St. Petersburg: Izd. SPbGPU, 2012 (in Russian).

Volume 9, Issue 4, 2018 



\title{
EFFECTS OF VISCOUS DISSIPATION AND HEAT GENERATION/ABSORPTION ON NANOFLUID FLOW OVER AN UNSTEADY STRETCHING SURFACE WITH THERMAL RADIATION AND THERMOPHORESIS
}

\author{
Alok Kumar Pandey ${ }^{1, *}$ E Manoj Kumar ${ }^{2}$ \\ ${ }^{1}$ Invertis University, Bareilly, 243123, Uttar Pradesh, India \\ ${ }^{2}$ Department of Mathematics, Statistics and Computer Science, G.B. Pant \\ University of Agriculture and Technology, Uttarakhand, 263145, India \\ *Address all correspondence to: Alok Kumar Pandey, Invertis University, Bareilly, \\ 243123, Uttar Pradesh, India, E-mail: mr.alokpandey1@gmail.com
}

Heat and mass transfer that exerts its influence on nanofluid flow through a heated stretching sheet with thermal radiation, viscous dissipation, and heat generation/absorption is analyzed. The transport model is influenced by Brownian motion and thermophoresis. The governing nonlinear PDEs are converted into a system of nonlinear ODEs by means of supplementary transformations and then handled numerically from the assisting RKF scheme of the 45th order with shooting procedure. The thermal field and concentration field are presented graphically for values of heat generation/absorption parameter, thermal radiation parameter, thermophoresis parameter, and Eckert number. The Nusselt number and Sherwood number are tabulated. The results of this study show that the heat transfer coefficient decreases with increase in the heat generation/absorption parameter and Eckert number, while the reverse trend is observed for mass transfer rate for the same pertinent parameters.

KEY WORDS: heat generation/absorption, stretching surface, thermal radiation, thermophoresis, viscous dissipation

\section{INTRODUCTION}

Over the past few decades, a lot of interest has been generated in boundary-layer flow over a stretching sheet due to its significant applications in engineering, for example, materials prepared by extrusion of plastic sheets, continuous cooling, fiber spinning, glass blowing, and so on. In these circumstances, the quality of products depends on the rate of heat transfer at the stretching sheet. Mahapatra and Gupta (2002) considered the heat transfer influence on stagnation-point flow of a 
viscous fluid over a stretched surface. The Keller box method was employed to get the governing equations of stagnation-point flow due to a stretching sheet given by Nazar et al. (2004) and Ishak et al. (2006). Vajravelu et al. (2011) applied the Keller box method to acquire the results of pertinent parameters of the problem on studying the flow of nanofluids due to a stretching surface. Das et al. (2014) examined the effect of unsteady boundary-layer flow together with radiation of nanofluid at a heated stretching sheet. Akbar et al. (2013) analyzed two-dimensional stagnation-point flow of an incompressible nanofluid towards a stretching surface. They calculated that the temperature profiles of nanoparticle volume fraction increases but the heat transfer rate decreases with increase in $N t$.

The influences of thermal radiation on flow and heat transfer have significant applications in engineering and physics, particularly, in space technology and hightemperature processes. The impact of thermal radiation also plays a vital role in controlling heat transfer in industry where the quality of final product depends on heat controlling factors to some extent. The effects of thermal radiation on different geometries were studied by Das et al. (2014), Kothandapani and Prakash (2015), Haq et al. (2015), and Akbar et al. (2013). Singh et al. (2016) investigated the influence of MHD slip flow on an alumina-water nanofluid due to a flat plate. They showed that the heat transfer rate increases with increase in the value of the volume fraction of alumina-water nanofluid. Pandey and Kumar (2017c) analyzed the combined influence of viscous dissipation and thermal raditation on $\mathrm{Cu}$-water nanofluid flow over a wedge surface. They described that the temperature profiles of the $\mathrm{Cu}-$ water nanofluid increase regularly along with the Eckert number. Mishra et al. (2018) explained the effects of viscous dissipation and suction/injection on Agwater nanofluid flow over a stretching cylinder. Pandey and Kumar (2018a) studied unsteady squeezing nanofluid flow between two parallel plates. Pandey and Kumar (2018b) analyzed the influence of MHD flow of nanofluid through a stretching/shrinking convergent/divergent channel with viscous dissipation. Pal and Mandal (2015) analyzed the impact of magnetohydrodynamic and viscous dissipation on fluid flow past a vertical stretching/shrinking sheet in cooperation with thermal radiation and Ohmic heating. Mabood et al. (2015) discussed the consequence of mass and heat transfer plus MHD flow of nanofluids past a nonlinear stretching surface numerically. Lin et al. (2015) inspected the influences of viscous dissipation and heat transfer in common with thermal conductivity of finite pseudoplastic nanofluid flow past an unsteady stretching surface. Freidoonimehr et al. (2015) discussed the impact of unsteady MHD free convection flow past a leaky stretchable vertical sheet in a nanofluid. MHD flow of nanofluid over a permeable stretching sheet in conjunction with heat generation/absorption was considered by Pandey and Kumar (2016b). Hakeem et al. (2015) investigated the influence of thermal radiation due to nanofluid flow past a stretchable/shrinkable sheet of second-order slip. Alsaedi et al. (2012) discussed stagnation-point nanofluid flow past a sheet due to heat generation/absorption effect along with convective boundary conditions. Nandy and Mahapatra (2013) studied two-dimensional MHD stagnation-point flow due to 
a stretchable/shrinkable sheet under the upshot of heat generation via viscous dissipation plus convective boundary conditions. Hayat et al. (2015) utilized the homotopy analysis method (HAM) to acquire the solution for mixed-convection flow of a Casson nanofluid due to a stretched surface. In several literatures, the influence of MHD flow of a working fluid due to various geometries was studied (Hayat et al., 2016; Haq et al., 2015; Jalilpour et al., 2014; Ibrahim and Shankar, 2013). The significance of viscous dissipation in fluid flow over different geometries was studied by Pandey and Kumar (2016a, 2017a,b) and Hayat et al. (2016). Kothandapani and Prakash (2015) studied peristaltic motion of a Williamson nanoliquid due to the thermal radiation and magnetic field effects in a tapered asymmetric channel.

Investigations of heat generation/absorption in moving fluids play a significant role in studying fluid flow and heat transfer rate. The effects of heat generation/absorption change on the temperature profiles due to this cause particle deposition that may arise in semi-conductor wafers, electronic chips, and nuclear reactors. Moshizi et al. (2014) theoretically investigated an alumina-water nanofluid inside a concentric pipe with heat generation/absorption. Upreti et al. (2017) analyzed combined effects of heat generation/absorption and viscous-Ohmic dissipation on porous flat plate using an Ag-water nanofluid. They illustrated that the temperature profile of Ag-water nanofluid is an increasing function of the heat generation/absorption parameter.

Thermophoresis occurs when high-energy molecules in a warmer region of liquid impinge on the molecule with greater momentum than molecules from a cold area. This leads to a movement of particles in the direction opposite to that of the temperature gradient, i.e., from warmer areas to cooler ones. There are several engineering applications of thermophoresis phenomenon in removing small particles from gas streams, in determining exhaust gas particle trajectories from combustion devices, and in studying the particulate material deposition on turbine blades. Loganathan and Arasu (2010) examined the impact of thermophoresis on viscous fluid flow over a porous wedge surface with suction/injection. Rahman et al. (2012) studied the influence of thermophoresis on unsteady convective heat and mass transfer flow over a wedge surface. Postelnicu (2012) investigated the combined effects of thermophoresis and natural convection over inclined surfaces. Singh et al. (2018) elucidated the impact of thermophoresis and Brownian motion on MHD nanofluid flow over a stretching sheet. They found that the concentration profile of a $\mathrm{Cu}$-water nanofluid is constantly growing with the thermophoresis parameter. The Brownian motion and thermophoresis influence on nanofluid flow on a stretching surface were proposed by Khan and Pop (2010).

The goal of the recent paper is to study the impact of heat generation/absorption together with viscous dissipation and thermal radiation on nanofluid flow owing to an unsteady stretching sheet. An assisting transformation is adopted to renovate the governing PDEs into a set of nonlinear ODEs and solutions are obtained with the aid of the fourth-fifth-order Runge-Kutta-Fehlberg technique via shooting proce-

Volume 9, Issue 4, 2018 
dure, and several graphical results are analyzed in detail with various employed parameters.

\section{MATHEMATICAL FORMULATION}

The geometry of the model together with flow arrangement is displayed in Fig. 1. The system deals with two-dimensional unsteady nanofluid flow past a heated stretchable surface in the presence of radiation, thermophoresis, and heat generation/absorption. The flow is supposed to be restricted to $y>0$. The two identical and reverse forces are spontaneously employed in the $x$ direction, in such a way that the sheet being stretched is fixed at the origin. The authors consider that both the fluid and heat flows are in steady condition when $t<0$. The unsteady flow and heat flow start from $t=0$. The stretching surface having the velocity $U_{w}(x, t)=(a x) /(1-\lambda t)$, down the $x$ axis, where $a>0$, is known as the stretching rate and where $\lambda>0$ is a constant. It is also obvious that the stretching rate $(a x) /(1-\lambda t)$ amplifies with time since $a>0$, the velocity of the ambient fluid along the $y$ axis is $U_{e}(x, t)=$ $b x /(1-\lambda t)$, where the stagnation flow strength is $b>0$. It is also assumed that the sheet temperature $T_{w}(x, t)$ and nanoparticle volume fraction $C_{w}(x, t)$ at the surface depend on the distance $x$ along the sheet and time $t$, respectively. It is presumed that there is no-slip among the suspended nanoparticles and a regular fluid due to thermal equilibrium.

Under the above assumptions, the basic unsteady conservation of mass, momentum, and energy and concentration equations of nanofluid in the presence of radiation, viscous dissipation, thermophoresis, and heat generation/absorption past a stretching sheet can be expressed as follows:

$$
\frac{\partial u}{\partial x}+\frac{\partial v}{\partial y}=0
$$

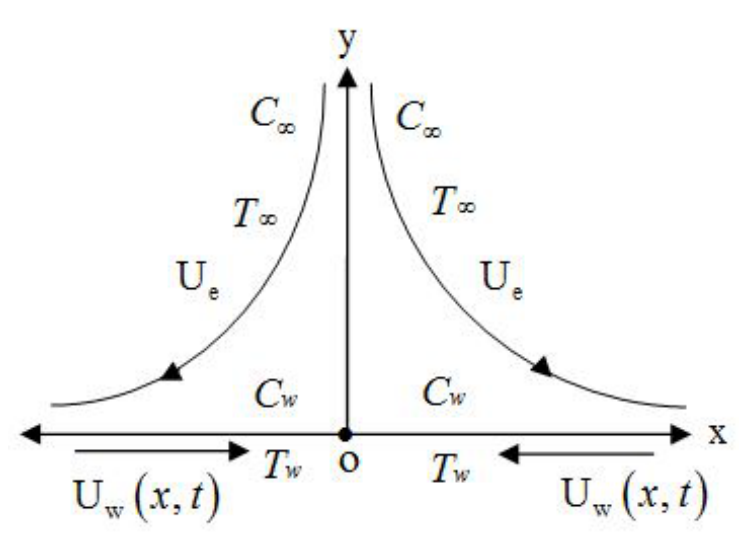

FIG. 1: Physical model and coordinate system 


$$
\begin{gathered}
\frac{\partial u}{\partial t}+u \frac{\partial u}{\partial x}+v \frac{\partial u}{\partial y}=\frac{\partial U_{e}}{\partial t}+U_{e} \frac{d U_{e}}{d x}+\frac{\mu_{n f}}{\rho_{n f}} \frac{\partial^{2} u}{\partial y^{2}}, \\
\frac{\partial T}{\partial t}+u \frac{\partial T}{\partial x}+v \frac{\partial T}{\partial y}=\alpha_{n f} \frac{\partial^{2} T}{\partial y^{2}} \\
+\tau\left[D_{B} \frac{\partial C}{\partial y} \frac{\partial T}{\partial y}+\frac{D_{T}}{T_{\infty}}\left(\frac{\partial T}{\partial x}\right)^{2}\right]+\frac{\mu_{n f}}{\left(\rho C_{p}\right)_{n f}}\left(\frac{\partial u}{\partial y}\right)^{2} \\
-\frac{1}{\left(\rho C_{p}\right)_{n f}} \frac{\partial q_{r}}{\partial y}+\frac{Q_{0}}{\left(\rho C_{p}\right)_{n f}}\left(T-T_{\infty}\right), \\
\frac{\partial C}{\partial t}+u \frac{\partial C}{\partial x}+v \frac{\partial C}{\partial y}=D_{B} \frac{d^{2} C}{d y^{2}}+\frac{D_{T}}{T_{\infty}} \frac{\partial^{2} T}{\partial y^{2}},
\end{gathered}
$$

where $u$ and $v$ are the components of velocity in the $x$ and $v$ direction, respectively, $\rho_{f}$ is the density of the base fluid, $\alpha_{n f}=\kappa_{n f} /\left(\rho C_{p}\right)_{n f}$ is the thermal diffusivity of the nanofluid fluid, $\tau=\left(\rho C_{p}\right)_{s p} /\left(\rho C_{p}\right)_{b f}$ is the ratio of heat capacity of nanoparticle to the heat capacity of the base fluid, $\left(\rho C_{p}\right)_{s p}$ and $\left(\rho C_{p}\right)_{b p}$ are the specific heat parameters of the solid nanoparticles and regular fluid, respectively, $T$ is the temperature of the fluid, $C$ is the solid nanoparticle volumetric fraction, $T_{\infty}$ and $C_{\infty}$ are the temperature and solid nanoparticle fraction of the fluid far from the sheet, $D_{B}$ is the Brownian diffusion coefficient, $D_{T}$ be the thermophoretic diffusion coefficient, and $q_{t}$ is radiative heat flux in the $y$ direction.

Employing the Rosseland approximation, the radiative heat flux can be written as

$$
\frac{\partial q_{r}}{\partial y}=-\frac{16 \sigma^{*} T_{0}^{3}}{3 \kappa^{*}} \frac{\partial^{2} T}{\partial y^{2}},
$$

where $\sigma^{*}$ is the Stefan-Boltzmann constant and $\kappa^{*}$ is the mean absorption coefficient.

Using Eq. (5), we obtain the energy equation (3) in the form

$$
\begin{gathered}
\frac{\partial T}{\partial t}+u \frac{\partial T}{\partial x}+v \frac{\partial T}{\partial y}=\alpha_{n f}\left(1+\frac{16 \sigma^{*} T_{\infty}^{3}}{3 \kappa_{n f} \kappa^{*}}\right) \frac{\partial^{2} T}{\partial y^{2}} \\
+\tau\left[D_{B} \frac{\partial C}{\partial y} \frac{\partial T}{\partial y}+\frac{D_{T}}{T_{\infty}}\left(\frac{\partial T}{\partial y}\right)^{2}\right]+\frac{\mu_{n f}}{\left(\rho C_{p}\right)_{n f}}\left(\frac{\partial u}{\partial y}\right)^{2}+\frac{Q_{0}}{\left(\rho C_{p}\right)_{n f}}\left(T-T_{\infty}\right),
\end{gathered}
$$


with the boundary conditions

$$
\left\{\begin{array}{ll}
u=U_{w}(x, t), \quad v=0, T=T_{w}, \quad C=C_{w} & \text { at } y \rightarrow 0 \\
u=U_{e}(x, t), \quad T=T_{\infty}, C=C_{\infty} & \text { as } y \rightarrow \infty
\end{array} .\right.
$$

The wall temperature $T_{w}(x, t)$ and the volume fraction of nanoparticles $C_{w}(x, t)$ are defined as

$$
\begin{gathered}
T_{w}(x, t)=T_{\infty}+T_{0}\left(\frac{a x^{2}}{2 v_{b f}}\right)(1-\lambda t)^{2}, \\
C_{w}(x, t)=C_{\infty}+C_{0}\left(\frac{a x^{2}}{2 v_{b t}}\right)(1-\lambda t)^{-2},
\end{gathered}
$$

where $\left(T_{\infty}, C_{\infty}\right)$ are the free stream and $\left(T_{0}, C_{0}\right)$ are the positive reference values of temperature and volume fraction of solid nanoparticles, respectively, such that $0<<T_{0}<<T_{w}$ and $0<<C_{0}<<C_{w}$. It is noted that the above expressions are appropriate for the time $t<\lambda^{-1}$.

The following nondimensional similarity transformations are invoked:

$$
\begin{gathered}
u=\frac{\partial \psi}{\partial y}, \quad v=-\frac{\partial \psi}{\partial x}, \quad u=\left(\frac{a x}{(1-\lambda t)}\right) f^{\prime}(\eta) \\
v=-\left(\frac{a v_{b f}}{(1-\lambda t)}\right)^{1 / 2} f(\eta), \quad \psi=\left(\frac{a v_{b f}}{(1-\lambda t)}\right)^{1 / 2} x f(\eta), \\
\eta=\left(\frac{a}{(1-\lambda t) v_{b f}}\right), \quad T=T_{\infty}+T_{0}\left(\frac{a x^{2}}{(1-\lambda t)^{-2}}\right) \theta \\
C=C_{\infty}+C_{0}\left(\frac{a x^{2}}{(1-\lambda t)^{-2}}\right) \phi .
\end{gathered}
$$

Now substituting Eqs. (9) into Eqs. (2), (4), and (6), respectively, we acquire the following system of coupled nonlinear ODEs:

$$
f^{\prime \prime \prime}+(f-0.5 \eta S) f^{\prime \prime}-f^{\prime}\left(f^{\prime}+S\right)+\beta^{2}+\beta S=0,
$$




$$
\begin{gathered}
\frac{1}{\operatorname{Pr}}(1+N r) \theta^{\prime \prime}-S\left(2 \theta+0.5 \eta \theta^{\prime}\right) \\
+f \theta^{\prime}-2 f^{\prime} \theta+N b \theta^{\prime} \phi^{\prime}+N t \theta^{\prime 2}+\operatorname{Ec} f^{\prime \prime 2}+H \theta=0, \\
\phi^{\prime \prime}-S\left(2 \phi+0.5 \eta \phi^{\prime}\right) \text { Le }+ \text { Le } f \phi^{\prime}+\frac{N t}{N b} \theta^{\prime \prime}=0 .
\end{gathered}
$$

Boundary conditions (7) converted in terms of $f, \theta$, and $\phi$, are expressed as

$$
\left\{\begin{array}{l}
f(0)=0, f^{\prime}(0)=1, \quad \theta(0)=1, \phi(0)=1, \\
f^{\prime}(\infty)=\beta, \quad \theta(\infty)=0, \quad \phi(\infty)=0 .
\end{array}\right.
$$

The nondimensional pertinent parameters are

$$
\begin{gathered}
s=\frac{\lambda}{a}, \quad \beta=\frac{b}{a}, \quad \operatorname{Pr}=\frac{v_{b f}}{\alpha_{b f}}, \quad \text { Le }=\frac{v_{b f}}{D_{B}}, N r=\frac{16 \sigma^{*} T_{\infty}^{3}}{3 \kappa^{*} \kappa_{n f}}, \\
\operatorname{Re}_{x}=\frac{U_{w} x}{v_{f}}, \quad N b=\frac{D_{B}\left(\rho C_{p}\right)_{s p}\left(C_{w}-C_{\infty}\right)}{v_{b f}\left(\rho C_{p}\right)_{b f}}, N t=\frac{D_{T}\left(\rho C_{p}\right)_{s p}\left(T_{w}-T_{\infty}\right)}{v_{b f} T_{\infty}\left(\rho C_{p}\right)_{b f}}, \\
\mathrm{Ec}=\frac{\mu_{n f}}{\left(T_{w}-T_{\infty}\right)\left(\rho C_{p}\right)_{n f}}\left(\frac{U_{w}^{2}}{2 v_{b f}^{2}(1-\lambda t)^{4}}\right), \text { and } H=\frac{(1-\lambda t) Q_{0}}{a\left(\rho C_{p}\right)_{n f}} .
\end{gathered}
$$

The expressions for the local Nusselt number $\mathrm{Nu}$ and the local Sherwood number Sh are defined as

$$
\begin{gathered}
\mathrm{Nu}=\frac{x}{\kappa\left(T_{w}-T_{\infty}\right)}\left[\kappa\left(\frac{\partial T}{\partial y}\right)_{y=0}-\frac{4 \sigma^{*}}{3 \kappa^{*}}\left(\frac{\partial T^{4}}{\partial y}\right)_{y=0}\right], \\
\mathrm{Sh}=-\frac{x}{\left(C_{w}-C_{\infty}\right)}\left(\frac{\partial C}{\partial y}\right)_{y=0} .
\end{gathered}
$$

Solving Eq. (14) with the aid of the above equations, we get

$$
-(1+N r) \theta^{\prime}(0)=\mathrm{Nu} r=\operatorname{Re}_{x}^{1 / 2} \mathrm{Nu},-\phi^{\prime}(0)=\mathrm{Sh} r=\operatorname{Re}_{x}^{1 / 2} \mathrm{Sh},
$$

where Nu $r$ is the reduced Nusselt number and Sh $r$ is the reduced Sherwood number. 


\section{METHOD OF SOLUTION}

The set of ordinary differential equations (10)-(12) subject to the associated boundary conditions (13) have been solved by the fourth-fifth-order RKF method together with a shooting technique. This scheme depends on the discretization of the problem domain and the computation of unidentified boundary conditions. The range of the study is discretized, and the boundary conditions for $\eta_{\infty}$ are exchanged by $f^{\prime}\left(\eta_{\infty}\right)=\beta, \theta\left(\eta_{\infty}\right)=0=\phi\left(\eta_{\infty}\right)$, where $\eta_{\infty}$ is the large value of the variable $\eta$ for boundary conditions (13). We take $\eta_{\infty}=3$ and mesh size $\Delta \eta=0.001$. To solve the problem, the nonlinear equations (10)-(12) have been renewed into eight first-order linear ODEs. There are four conditions at the boundary $\eta=0$ and three conditions at the boundary $\eta=\infty$. To acquire the outcomes of the problem, we should have three more conditions like $f^{\prime \prime}\left(\eta_{0}\right), \theta^{\prime}\left(\eta_{0}\right)$, and $\phi^{\prime}\left(\eta_{0}\right)$. These conditions have been obtained by the shooting technique.

\section{CODE VALIDATION}

In order to certify the accurateness of our code, we compared our results for the skin friction coefficient at diverse values of the stretching parameter $\beta$ with those obtained by Mahapatra and Gupta (2002), Nazar et al. (2004), Ishak et al. (2006), and Das et al. (2014) and found that the results are in excellent agreement, as is seen from Table 1. Hence employing the present code is justified.

\section{RESULTS AND DISCUSSION}

The effects of several objective parameters on $\theta(\eta)$ and $\phi(\eta)$ are examined with the aid of graphs, and the influence of these parameters on the rate of heat and mass transfer is also revealed. Table 2 displays the coefficient of heat and mass transfer for of the selected values of the Eckert number Ec, heat generation/absorption parameter $H$, thermal radiation parameter $N r$, and thermophoresis parameter Nt. From the table it is detected that the heat transfer rate decreased with increase in the numerical values of the heat generation/absorption parameter, Eckert number,

TABLE 1: Comparison of skin friction coefficient $f$ "(0) when $\operatorname{Pr}=1$

\begin{tabular}{|c|c|c|c|c|c|}
\hline $\boldsymbol{\beta}$ & $\begin{array}{c}\text { Mahapatra and } \\
\text { Gupta (2002) }\end{array}$ & $\begin{array}{c}\text { Nazar et al. } \\
\mathbf{( 2 0 0 4 )}\end{array}$ & $\begin{array}{c}\text { Ishak et al. } \\
\mathbf{( 2 0 0 6 )}\end{array}$ & $\begin{array}{c}\text { Das et al. } \\
\mathbf{( 2 0 1 4 )}\end{array}$ & $\begin{array}{c}\text { Present } \\
\text { Study }\end{array}$ \\
\hline 0.1 & -0.9694 & -0.9694 & -0.9694 & -0.969328 & -0.9694 \\
\hline 0.2 & -0.9181 & -0.9181 & -0.9181 & -0.9180980 & -0.918121 \\
\hline 0.5 & -0.6673 & -0.6673 & -0.6673 & -0.667301 & -0.667283 \\
\hline 2.0 & 2.0175 & 2.0176 & 2.0175 & 2.017467 & 2.017394 \\
\hline 3.0 & 4.7293 & 4.7296 & 4.7294 & 4.729406 & 4.7290374 \\
\hline
\end{tabular}


TABLE 2: Several values of Nusselt number $(-\theta(0))$ and Sherwood number $(-\phi(0))$ at $\beta=0.5$, Le $=1, N b=0.1, \operatorname{Pr}=0.72$, and $S=0.4$

\begin{tabular}{|c|c|c|c|c|c|}
\hline $\boldsymbol{H}$ & $\boldsymbol{N r}$ & $\boldsymbol{N t}$ & $\mathbf{E c}$ & $-\boldsymbol{\theta ( \mathbf { 0 } )}$ & $-\boldsymbol{\phi ( \mathbf { 0 } )}$ \\
\hline-1 & 0.1 & 0.1 & 0.03 & 1.5208607 & 0.509264 \\
\hline 0 & - & - & - & 1.2908998 & 0.686028 \\
\hline 1 & - & - & - & 1.0081 & 0.890065 \\
\hline 2 & - & - & - & 0.611253 & 1.1471157 \\
\hline- & 0 & - & 0.1 & 0.6295 & 1.137933 \\
\hline- & 0.3 & - & - & 0.56455 & 1.17568 \\
\hline- & 0.5 & - & - & 0.534967 & 1.192782 \\
\hline- & 1 & - & - & 0.4859567 & 1.220976 \\
\hline 1 & 0.5 & 0.3 & - & 0.8376321 & 0.3979916 \\
\hline- & - & 0.5 & - & 0.81525452 & -0.13403528 \\
\hline- & - & 0.8 & - & 0.7835876 & -0.8332672 \\
\hline- & - & - & 0.01 & 1.0099051 & 0.888593 \\
\hline- & - & - & 0.1 & 1.0018079 & 0.895227 \\
\hline- & - & - & 0.6 & 0.956824 & 0.9320958 \\
\hline- & - & - & 1 & 0.920834 & 0.961597 \\
\hline- & - & - & 5 & 0.560631 & 1.256861 \\
\hline
\end{tabular}

and radiation parameter, while the mass transfer rate was constantly enhanced in the same cases and at the same parameters. The graph of nondimensional temperature and concentration profiles of nanofluid are influenced by pertinent parameters as revealed in Figs. 2-9. The heat generation/absorption parameter $H$ exerts its influence on the temperature and concentration profiles at $\operatorname{Pr}=0.72$, Ec $=0.03, N b=N r=N t=0.1, S=0.4, \beta=0.5$, and Le $=1$ as depicted in Figs. 2 and 3, respectively. In Fig. 2, the thermal field profiles of the nanofluid regularly accelerated with heat generation/absorption corresponding to each value of the similarity variable $\eta$. Moreover, the thermal boundary layer also increases with $H$. Figure 3 reveals variation of the concentration curves with the dimensionless parameter $\eta$ for several values of $H$. In these graphs, we examined two cases, firstly the concentration of the nanofluid as an accelerating function of $H$ in the fixed range of $\eta \in[0,0.25)$; in this section the thermal boundary layer decreases when the heat generation/absorption parameter $H$ increases. In the second case, the trend is opposite in the domain of $\eta \in[0.25,3)$, it means that the concentration profiles increase slowly with $H$. The temperature and concentration graphs of the nanofluid for several values of radiation parameter $\mathrm{Nr}$ are presented in Figs. 4 and 5, respectively, when $\operatorname{Pr}=0.72, \mathrm{Ec}=0.03, N b=N t=0.1, H=2, S=0.4, \beta=0.5$, and 


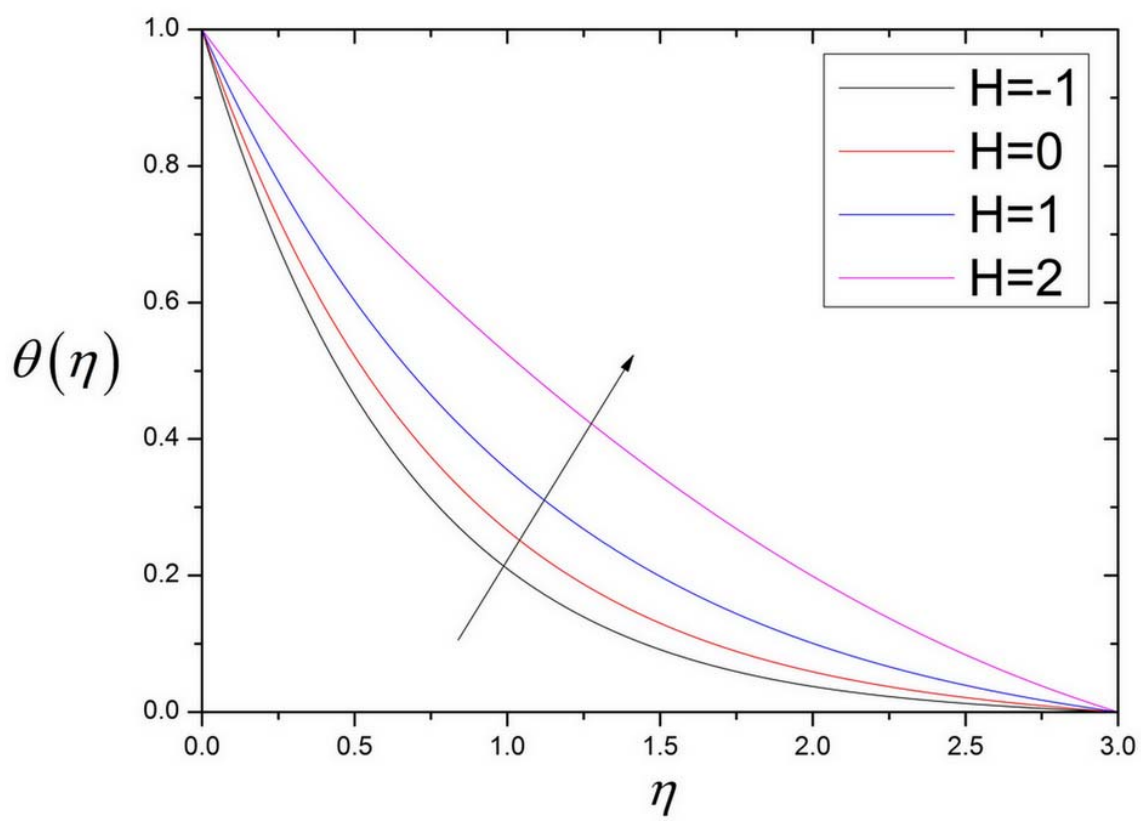

FIG. 2: Influence of $H$ on temperature profiles

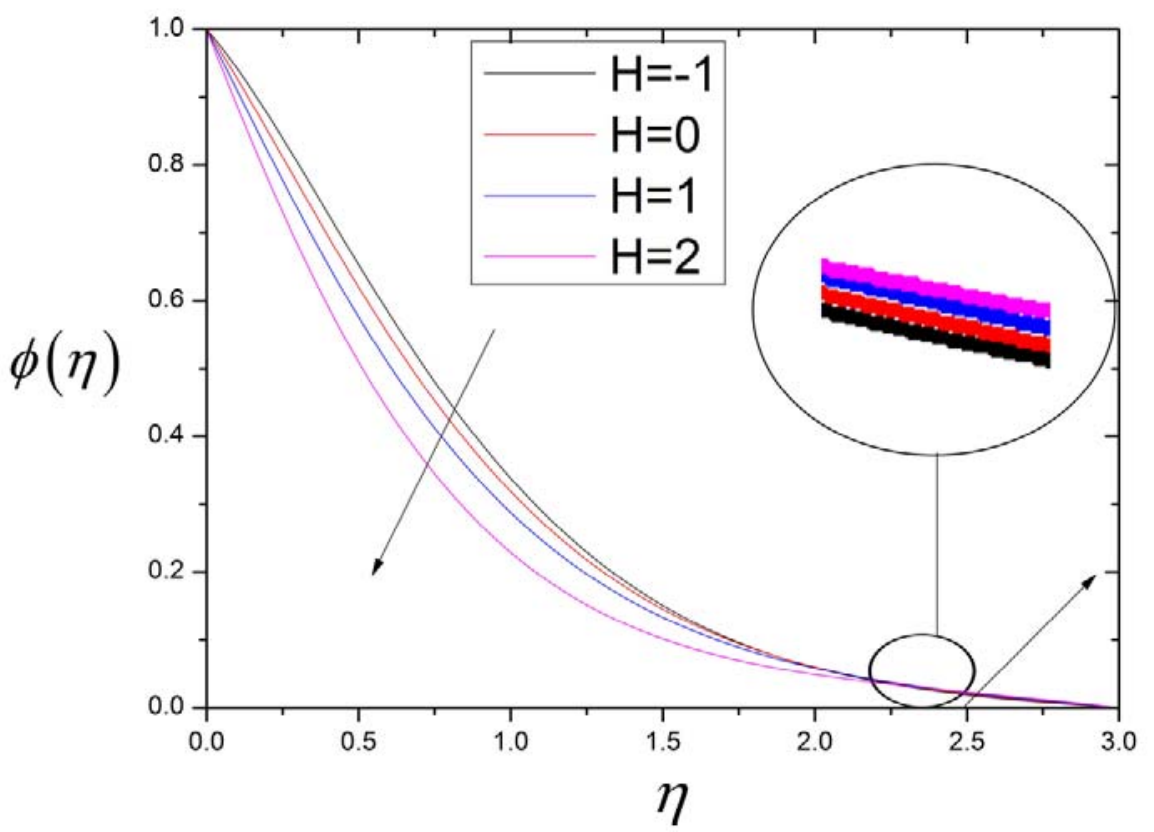

FIG. 3: Influence of $H$ on concentration profiles 


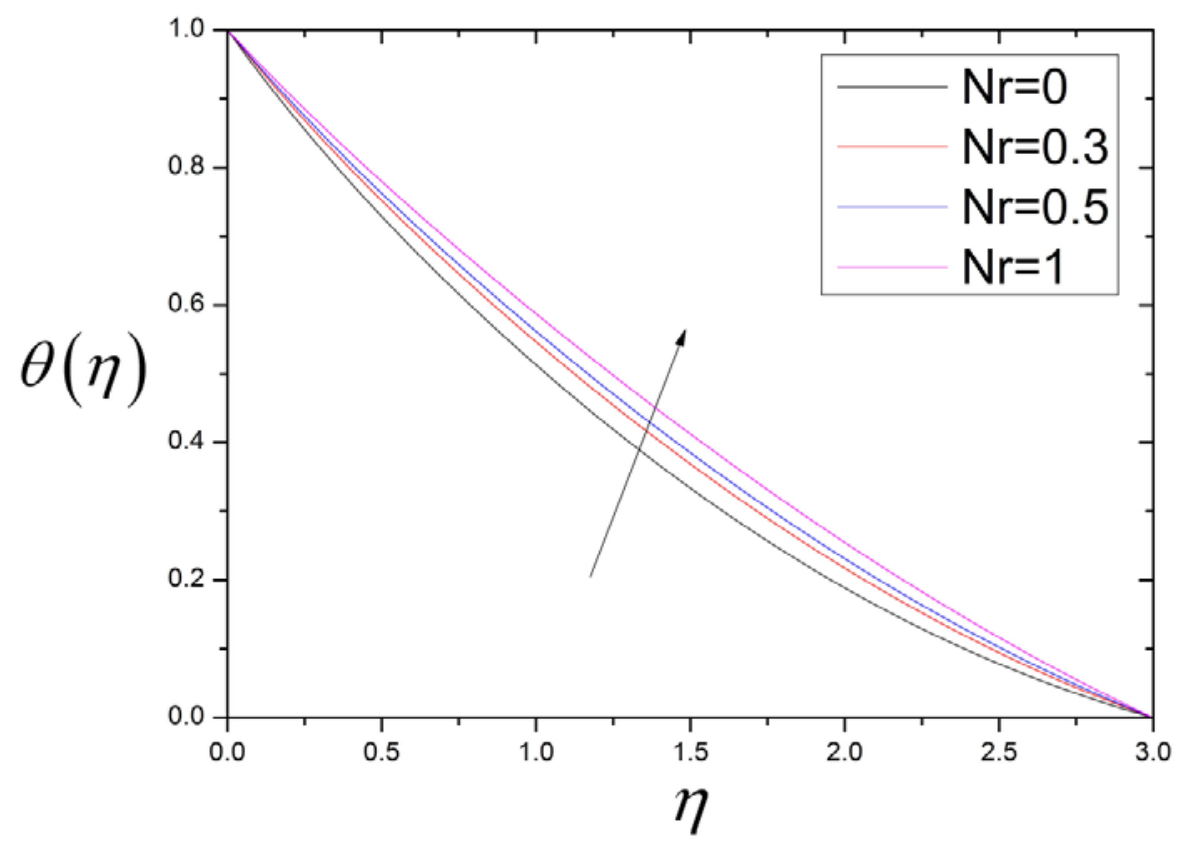

FIG. 4: Influence of $\mathrm{Nr}$ on temperature profiles

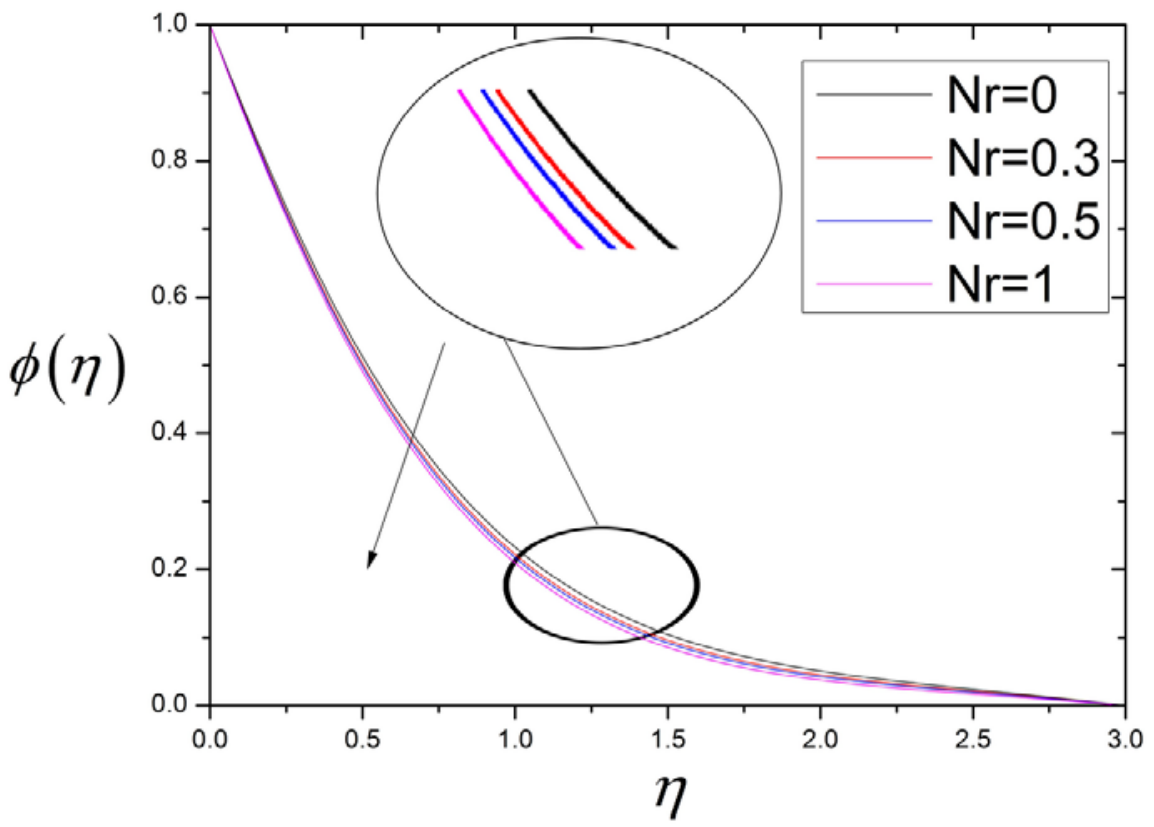

FIG. 5: Influence of $\mathrm{Nr}$ on concentration profiles 


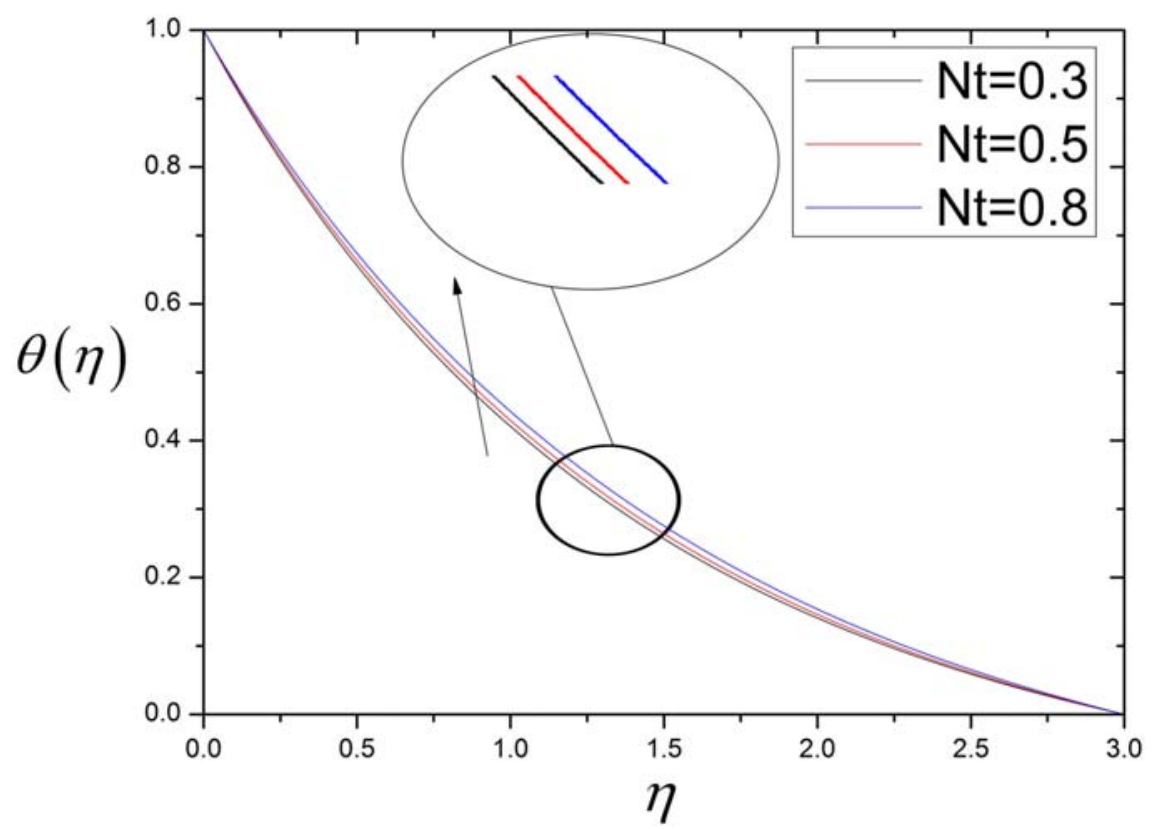

FIG. 6: Influence of $N t$ on temperature profiles

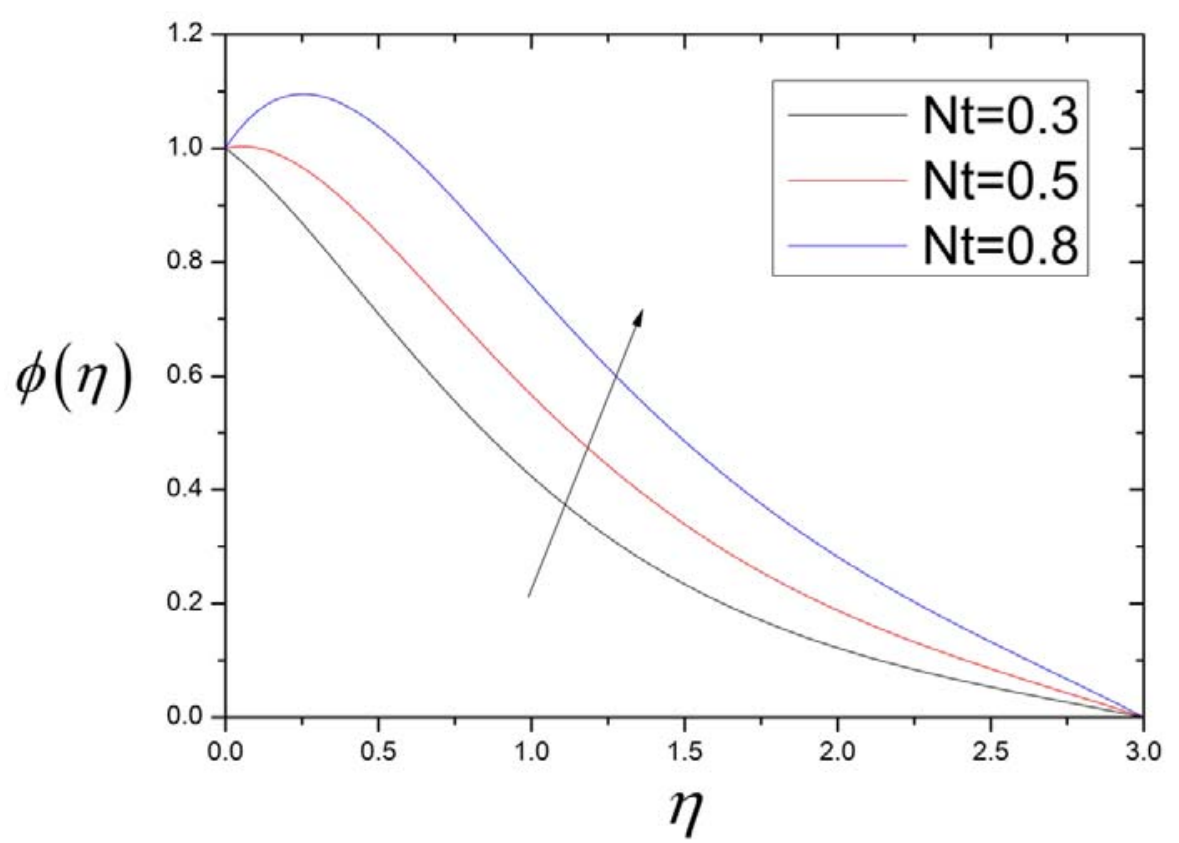

FIG. 7: Influence of $N t$ on concentration profiles 


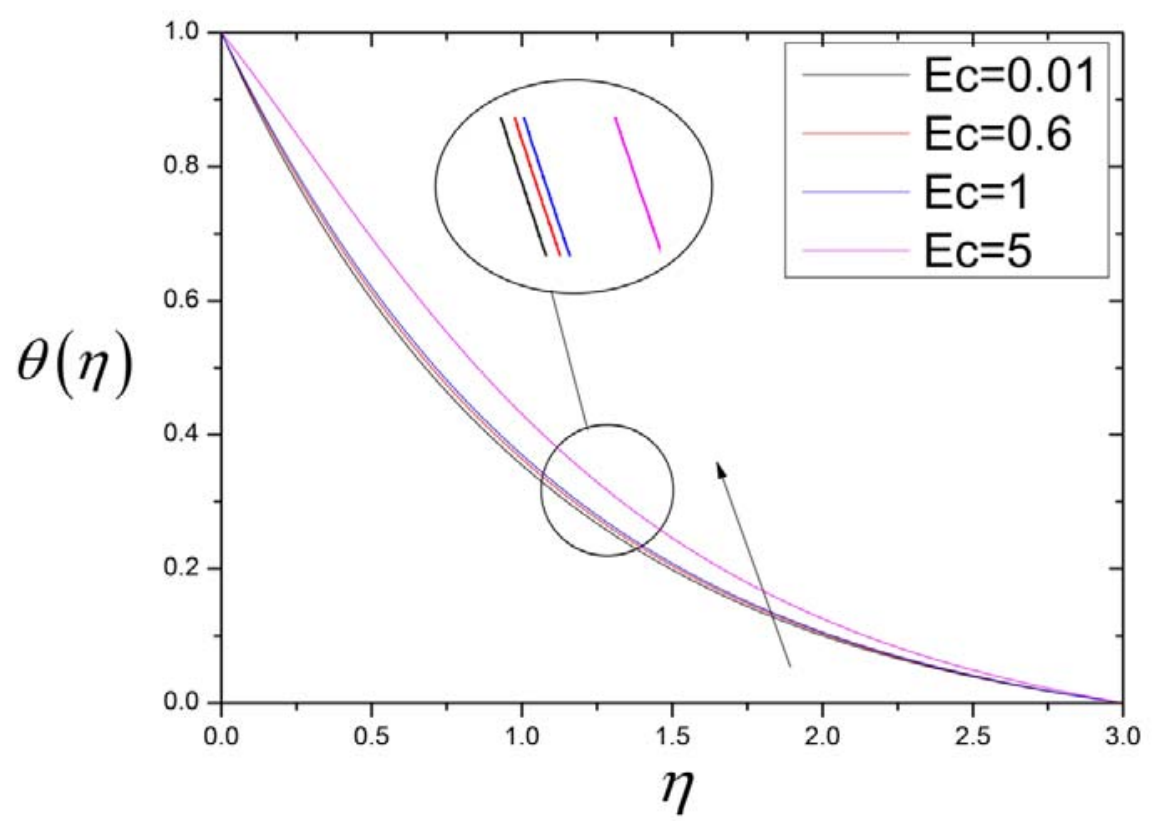

FIG. 8: Influence of Ec on temperature profiles

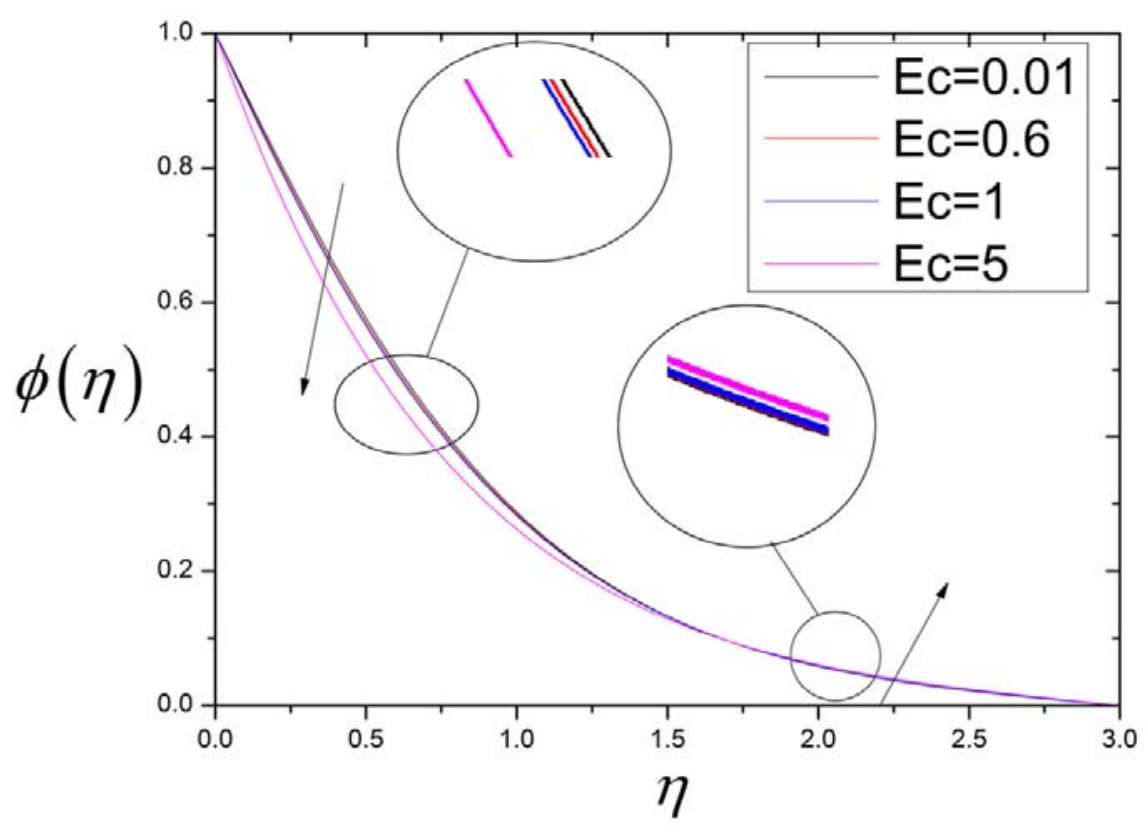

FIG. 9: Influence of Ec on concentration profiles 
$\mathrm{Le}=1$. Figure 4 displays the influence of the radiation parameter $\mathrm{Nr}$ on the thermal field. As the radiation parameter increases in the range $\eta \in[0,3]$, the thermal boundary layer is endlessly amplified. Figure 5 shows the asymptotic behavior of the concentration profiles of nanofluid for a variety of values of the radiation parameter $\mathrm{Nr}$. In analyzing these graphs, we saw that the concentration curves were continuously reduced with increase in the values of the thermal radiation parameter $N r$. However, the concentration boundary-layer thickness of the nanofluid decreased promptly with increase in $N r$. Figures 6 and 7 have been sketched to examine the variation in the thermal and concentration curves of the nanofluid with $\eta$ for unlike values of the thermophoresis parameter $N t$ at $\operatorname{Pr}=0.72, \mathrm{Ec}=0.1, N b=N t=0.1$, $N r=0.5, H=1, S=0.4, \beta=0.5$, and $\mathrm{Le}=1$. It is noticed that the temperature and concentration curves are augmented on increase in the values of Nt. Moreover, the concentration and thermal boundary-layer width increase with Nt. In Table 2, we observed that as the values of $N t$ increased, the heat transfer rate decreased but the mass transfer rate increased rapidly with the thermal radiation parameter $N t$. The Eckert number Ec exerts its influence on the temperature and concentration profiles at $\operatorname{Pr}=0.72, N b=N r=N t=0.1, S=0.4, \beta=0.5$, and $\mathrm{Le}=H=1$, as depicted in Figs. 8 and 9, respectively. Figure 8 displays that the temperature of the nanofluid amplified with increase in Ec. Corresponding to each value of the dimensionless variable $\eta$, in the particular range $(0 \leq \eta \leq 3)$, the thermal boundary-layer thickness of the nanofluid increased. Figure 9 reveals that the concentration distribution profile of the nanofluid decreases with increase in the Eckert number Ec, when the value of the horizontal axis ( $\eta$ axis) lies in the range $0 \leq \eta<2$, however, the thickness of the thermal boundary layer decreases with increase in the Eckert number Ec. When the similarity variable lies in the range $2 \leq \eta<3$, the thermal field graphs trend changes gently and the thermal boundary-layer width also increases with Ec.

\section{CONCLUSIONS}

The solution for unsteady nanofluid flow over a stretching surface due to the influences of thermal radiation, thermophoresis, heat generation/absorption, and viscous dissipation has been examined numerically with the aid of the fourth-fifthorder RKF technique via the shooting technique. From the numerical experiments the authors arrived at the following conclusions:

- On increase in the thermophoresis parameter, both heat and mass transfer rates are reduced.

- The thermal flow field is enhanced continuously with heat generation/absorption parameter.

- The concentration profiles of nanofluid decrease with boosting in the radiation parameter.

- The thermal boundary-layer thickness increases with the Eckert number. 
- The Sherwood number increases with the heat generation/absorption parameter, while the Nusselt number decreases with rise in the heat generation/absorption parameter.

- The concentration boundary-layer width increases with the thermophoresis parameter.

\section{REFERENCES}

Akbar, N.S., Nadeem, S., Haq, R.U., and Khan, Z.H., Radiation Effects on MHD Stagnation Point Flow of Nano Fluid towards a Stretching Surface with Convective Boundary Condition, Chinese J. Aeronaut., vol. 26, no. 6, pp. 1389-1397, 2013.

Alsaedi, A., Awais, M., and Hayat, T., Effects of Heat Generation/Absorption on Stagnation Point Flow of Nanofluid over a Surface with Convective Boundary Conditions, Commun. Nonlinear Sci. Numer. Simul., vol. 17, no. 11, pp. 4210-4223, 2012.

Das, K., Duari, P.R., and Kundu, P.K., Nanofluid Flow over an Unsteady Stretching Surface in Presence of Thermal Radiation, Alexandria Eng. J., vol. 53, no. 3, pp. 737-745, 2014.

Freidoonimehr, N., Rashidi, M.M., and Mahmud, S., Unsteady MHD Free Convective Flow past a Permeable Stretching Vertical Surface in a Nano-Fluid, Int. J. Therm. Sci., vol. 87, pp. 136-145, 2015.

Hakeem, A.A., Ganesh, N.V., and Ganga, B., Magnetic Field Effect on Second Order Slip Flow of Nanofluid over a Stretching/Shrinking Sheet with Thermal Radiation Effect, J. Magn. Magn. Mater., vol. 381, pp. 243-257, 2015.

Haq, R.U., Nadeem, S., Khan, Z.H., and Akbar, N.S., Thermal Radiation and Slip Effects on MHD Stagnation Point Flow of Nanofluid over a Stretching Sheet, Phys. E: Low-dimens. Syst. Nanostr., vol. 65, pp. 17-23, 2015.

Hayat, T., Ashraf, M.B., Shehzad, S.A., and Alsaedi, A., Mixed Convection Flow of Casson Nanofluid over a Stretching Sheet with Convectively Heated Chemical Reaction and Heat Source/Sink, J. Appl. Fluid Mech., vol. 8, no. 4, pp. 803-813, 2015.

Hayat, T., Khan, M.I., Waqas, M., Yasmeen, T., and Alsaedi, A., Viscous Dissipation Effect in Flow of Magnetonanofluid with Variable Properties, J. Molec. Liq., vol. 222, pp. 47-54, 2016.

Ibrahim, W. and Shankar, B., MHD Boundary Layer Flow and Heat Transfer of a Nanofluid past a Permeable Stretching Sheet with Velocity, Thermal and Solutal Slip Boundary Conditions, Comput. Fluids, vol. 75, pp. 1-10, 2013.

Ishak, A., Nazar, R., and Pop, I., Mixed Convection Boundary Layers in the Stagnation-Point Flow toward a Stretching Vertical Sheet, Meccanica, vol. 41, no. 5, pp. 509-518, 2006.

Jalilpour, B., Jafarmadar, S., Ganji, D.D., Shotorban, A.B., and Taghavifar, H., Heat Generation/Absorption on MHD Stagnation Flow of Nanofluid towards a Porous Stretching Sheet with Prescribed Surface Heat Flux, J. Molec. Liq., vol. 195, pp. 194-204, 2014.

Khan, U., Ahmed, N., Asadullah, M., and Mohyud-din, S.T., Effects of Viscous Dissipation and Slip Velocity on Two-Dimensional and Axisymmetric Squeezing Flow of $\mathrm{Cu}-\mathrm{Water}$ and $\mathrm{Cu}-\mathrm{Kerosene}$ Nanofluids, Propuls. Power Res., vol. 4, no. 1, pp. 40-49, 2015.

Khan, W.A. and Pop, I., Boundary-Layer Flow of a Nanofluid past a Stretching Sheet, Int. J. Heat Mass Transf., vol. 53, no. 11, pp. 2477-2483, 2010.

Kothandapani, M. and Prakash, J., Effects of Thermal Radiation Parameter and Magnetic Field on the Peristaltic Motion of Williamson Nanofluids in a Tapered Asymmetric Channel, Int. J. Heat Mass Transf., vol. 81, pp. 234-245, 2015.

Volume 9, Issue 4, 2018 
Lin, Y., Zheng, L., and Chen, G., Unsteady Flow and Heat Transfer of Pseudo-Plastic Nanoliquid in a Finite Thin Film on a Stretching Surface with Variable Thermal Conductivity and Viscous Dissipation, Powder Technol., vol. 274, pp. 324-332, 2015.

Loganathan, P. and Arasu, P.P., Thermophoresis Effects on Non-Darcy MHD Mixed Convective Heat and Mass Transfer past a Porous Wedge in the Presence of Suction/Injection, Theor. Appl. Mech., vol. 37, no. 3, pp. 203-227, 2010.

Mabood, F., Khan, W.A., and Ismail, A.M., MHD Boundary Layer Flow and Heat Transfer of Nanofluids over a Nonlinear Stretching Sheet: A Numerical Study, J. Magn. Magn. Mater., vol. 374, pp. 569-576, 2015.

Mahapatra, T.R. and Gupta, A.S., Heat Transfer in Stagnation-Point Flow towards a Stretching Sheet, Heat Mass Transf., vol. 38, no. 6, pp. 517-521, 2002.

Mishra, A., Pandey, A.K., and Kumar, M., Ohmic-Viscous Dissipation and Slip Effects on Nanofluid Flow over a Stretching Cylinder with Suction/Injection, Nanosci. Technol.: An Int. J., vol. 9, no. 2, pp. 99-115, 2018.

Moshizi, S.A., Malvandi, A., Ganji, D.D., and Pop, I., A Two-Phase Theoretical Study of $\mathrm{Al}_{2} \mathrm{O}_{3}-$ Water Nanofluid Flow inside a Concentric Pipe with Heat Generation/Absorption, Int. J. Therm. Sci., vol. 84, pp. 347-357, 2014.

Nandy, S.K. and Mahapatra, T.R., Effects of Slip and Heat Generation/Absorption on MHD Stagnation Flow of Nanofluid past a Stretching/Shrinking Surface with Convective Boundary Conditions, Int. J. Heat Mass Transf., vol. 64, pp. 1091-1100, 2013.

Nazar, R., Amin, N., Filip, D., and Pop, I., Unsteady Boundary Layer Flow in the Region of the Stagnation Point on a Stretching Sheet, Int. J. Eng. Sci., vol. 42, no. 11, pp. 1241-1253, 2004.

Pal, D. and Mandal, G., Hydromagnetic Convective-Radiative Boundary Layer Flow of Nanofluids Induced by a Nonlinear Vertical Stretching/Shrinking Sheet with Viscous-Ohmic Dissipation, Powder Technol., vol. 279, pp. 61-74, 2015.

Pandey, A.K. and Kumar, M., Boundary Layer Flow and Heat Transfer Analysis on Cu-Water Nanofluid Flow over a Stretching Cylinder with Slip, Alexandria Eng. J., vol. 56, no. 4, pp. 671-677, 2017a.

Pandey, A.K. and Kumar, M., Chemical Reaction and Thermal Radiation Effects on Boundary Layer Flow of Nanofluid over a Wedge with Viscous and Ohmic Dissipation, St. Petersburg Polytech. Univ. J.: Phys. Math., vol. 3, no. 4, pp. 322-332, $2017 \mathrm{~b}$.

Pandey, A.K. and Kumar, M., Effect of Viscous Dissipation and Suction/Injection on MHD Nanofluid Flow over a Wedge with Porous Medium and Slip, Alexandria Eng. J., vol. 55, no. 4, pp. 3115-3123, 2016a.

Pandey, A.K. and Kumar, M., Impact of Heat Generation/Absorption on Steady MHD Boundary Layer Flow of Nanofluid over a Permeable Stretching Sheet in Porous Medium with Chemical Reaction, Int. J. Res. Appl. Sci. Eng. Technol, vol. 4, no. 1, pp. 138-141, 2016b.

Pandey, A.K. and Kumar, M., Natural Convection and Thermal Radiation Influence on Nanofluid Flow over a Stretching Cylinder in a Porous Medium with Viscous Dissipation, Alexandria Eng. J., vol. 56, no. 1, pp. 55-62, 2017c.

Pandey, A.K. and Kumar, M., Squeezing Unsteady MHD Cu-Water Nanofluid Flow between Two Parallel Plates in Porous Medium with Suction/Injection, Comput. Appl. Math. J., vol. 4, no. 2, pp. 31-42, 2018a. 
Pandey, A.K. and Kumar, M., MHD Flow inside a Stretching/Shrinking Convergent/Divergent Channel with Heat Generation/Absorption and Viscous-Ohmic Dissipation Utilizing Cu-Water Nanofluid, Comput. Therm. Sci.: An Int. J., vol. 10, no. 5, pp. 457-471, 2018 b.

Postelnicu, A., Thermophoresis Particle Deposition in Natural Convection over Inclined Surfaces in Porous Media, Int. J. Heat Mass Transf., vol. 55, pp. 2087-2094, 2012.

Rahman, A.T.M.M., Alam, M.S., and Chowdhury, M.K., Local Similarity Solutions for Unsteady Two-Dimensional Forced Convective Heat and Mass Transfer Flow along a Wedge with Thermophoresis, Int. J. Appl. Math. Mech., vol. 8, no. 8, pp. 86-112, 2012.

Rawat, S.K., Pandey, A.K., and Kumar, M., Effects of Chemical Reaction and Slip in the Boundary Layer of MHD Nanofluid Flow through a Semi-Infinite Stretching Sheet with Thermophoresis and Brownian Motion: The Lie Group Analysis, Nanosci. Technol.: An Int. J., vol. 9, no. 1, pp. 47-68, 2018.

Singh, P., Pandey, A.K., and Kumar, M., Forced Convection in MHD Slip Flow of Alumina Water Nanofluid over a Flat Plate, J. Enhanced Heat Transf., vol. 23, no. 6, pp. 487-497, 2016.

Upreti, H., Pandey, A.K., and Kumar, M., MHD Flow of Ag-Water Nanofluid over a Flat Porous Plate with Viscous-Ohmic Dissipation, Suction/Injection and Heat Generation/Absorption, Alexandria Eng. J., 2017. DOI: 10.1016/j.aej.2017.03.018

Vajravelu, K., Prasad, K.V., Lee, J., Lee, C., Pop, I., and Van Gorder, R.A., Convective Heat Transfer in the Flow of Viscous Ag-Water and $\mathrm{Cu}-$ Water Nanofluids over a Stretching Surface, Int. J. Therm. Sci., vol. 50, no. 5, pp. 843-851, 2011.

Volume 9, Issue 4, 2018 



\section{MHD MIXED CONVECTION IN TRAPEZOIDAL ENCLOSURES FILLED WITH MICROPOLAR NANOFLUIDS}

Sameh E. Ahmed, ${ }^{1,2}$ Ahmed Kadhim Hussein, ${ }^{3}$
M.A. Mansour, ${ }^{4}$ Z.A. Raizah, ${ }^{2,}{ }^{5}$ E Xiaohui Zhang

${ }^{1}$ Department of Mathematics, South Valley University, Faculty of Science, Qena, Egypt

${ }^{2}$ Department of Mathematics, Faculty of Science, Abha, King Khalid University, Saudi Arabia

${ }^{3}$ College of Engineering ,Mechanical Engineering Department, Babylon University, Babylon City, Hilla, Iraq

${ }^{4}$ Department of Mathematics, Assuit University, Faculty of Science, Assuit, Egypt

${ }^{5}$ College of Physics, Optoelectronics and Energy, Soochow University, Suzhou, China

*Address all correspondence to: Z.A. Raizah, Department of Mathematics, Faculty of Science, Abha, King Khalid University, Saudi Arabia, E-mail: zaalrazh@kku.edu.sa

A steady laminar two-dimensional magnetohydrodynamic mixed-convection flow in trapezoidal enclosures filled with water-based micropolar nanofluids is investigated numerically by using the finite difference method. The left and right inclined vertical sidewalls of the trapezoidal enclosure are maintained at a low temperature. The horizontal top wall is considered adiabatic and moves at a uniform lid-driven velocity, while a part of the bottom wall is subjected to a uniform heat source and the remaining parts of it are considered adiabatic. An external magnetic field at different orientation angles is applied on the left sidewall of the enclosure. The fluid inside the enclosure is a water-based micropolar nanofluid containing different types of solid spherical nanoparticles ( $\mathrm{Cu}, \mathrm{Ag}, \mathrm{Al}_{2} \mathrm{O}_{3}$, and $\mathrm{TiO}_{2}$ ). Parametric studies of the influence of various parameters such as the Hartmann number, Richardson number, the type of nanofluid, magnetic field orientation angle, dimensionless viscosity, dimensionless length, location of a heat source, solid volume fraction in the fluid flow, and the heat transfer rate have been conducted. Comparisons with previously published numerical works are performed and good agreement between the results is obtained. It is found that the average Nusselt number increases when the Richardson number decreases and the solid volume fraction increases, while it decreases as the heat source length increases. Moreover, it is observed that the Hartmann number, heat source location, and the dimensionless viscosity have a significant effect on the average Nusselt number.

KEY WORDS: mixed convection, magnetohydrodynamics, micropolar nanofluid, trapezoidal enclosure, lid-driven flow 


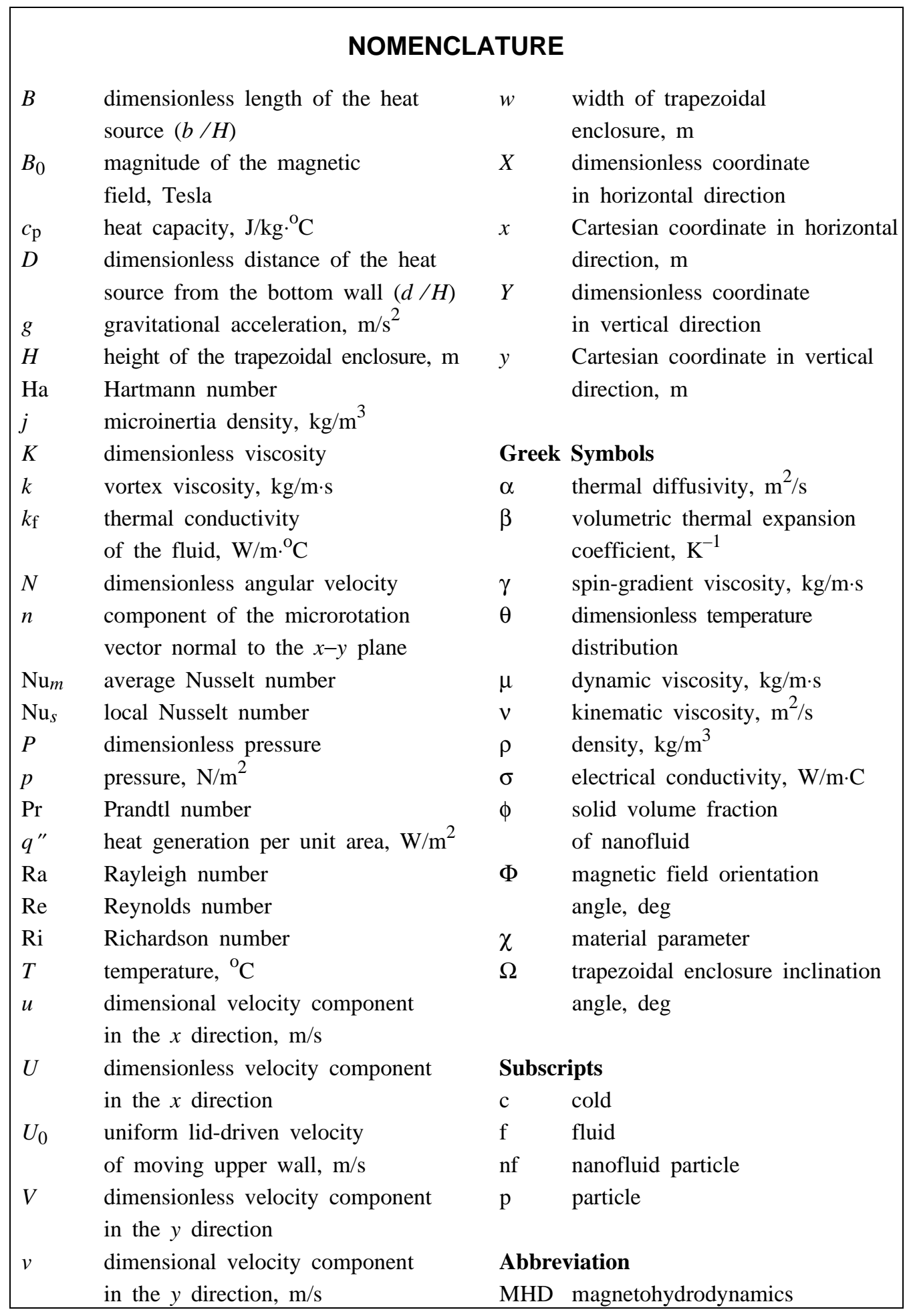




\section{INTRODUCTION}

Nanotechnology has been widely used in industry since materials with sizes of nanometers have clear physical and chemical properties. A fluid with added nanoscale particles is called a nanofluid which was firstly utilized by Choi (1995). Nanofluids are a new kind of heat transfer fluids containing a small quantity of nanosized particles (usually less than $100 \mathrm{~nm}$ ) uniformly and stably suspended in a liquid. They have received more attention as a new generation of heat transfer fluids in building heating, heat exchangers, plants and automotive cooling applications, because of their excellent thermal performance. Many studies indicate that the dispersion of a few amount of solid nanoparticles in conventional fluids remarkably increases their thermal conductivity. Compared to the existing techniques of enhancing heat transfer, the nanofluids show a superior potential for increasing heat transfer rates in a variety of cases. Various advantages of nanofluids applications include: improved heat transfer, heat transfer system size reduction, microchannel cooling and miniaturization of systems (Hussein, 2015; Kolsi et al., 2014). On the other hand, mixed convection in closed enclosures is one of the most important subjects of fluid flow and heat transfer. This subject has received much attention in the last ten years due to many energy-related applications, such as cooling of electronic components, thermal insulation of buildings using air gaps, solar energy collectors, dynamics of lakes, float glass production, and fire control in buildings (Hussain and Hussein, 2011). In fact, analysis of natural- or forcedconvection flows may be not so complex when they are investigated separately. But, when a temperature gradient is imposed so that shear-driven flow due to the lid-driven motion and natural-convection flow due to buoyancy effects are of comparable magnitude, the resulting flow falls in a mixed-convection regime, and the interaction and coupling of these effects make the analysis more complex. Numerous investigations have been conducted in the past on a lid-driven enclosure flow and heat transfer, considering various combinations of imposed temperature gradients and enclosure configurations (Sivasankaran et al., 2014). Most authors considered the flow and heat transfer inside a square or rectangular enclosure because the numerical solution is not complicated and the geometry is very simple to simulate. But, mixed convection in nonrectangular enclosures, such as triangular and trapezoidal ones, has received little attention even in the recent time, since it is much more complicated to investigate flow inside these enclosures. Also, actual or real enclosures occurring in practice generally have shapes different from a square or rectangle. Previous studies related to mixed-convection heat transfer in trapezoidal enclosures filled with a pure fluid are reported in literature by Tmartnhad et al. (2008), Hasib et al. (2015), Mamun et al. (2010), and Bhattacharya et al. (2013), whereas other authors investigated mixed-convection heat transfer in classical square or rectangular enclosures filled with a nanofluid. Sebdani et al. (2012) numerically investigated the problem of mixed-convection fluid flow and heat transfer of $\mathrm{Al}_{2} \mathrm{O}_{3}$-water nanofluid with temperature and nanoparticles concentration depend-

Volume 9, Issue 4, 2018 
ent on thermal conductivity and effective viscosity inside a square cavity with a heat source on the bottom wall, insulated top wall, and moving downward cold sidewalls. It was found that when a heat source was located in the middle of the bottom wall and the Rayleigh number was kept constant, the effect of the addition of nanoparticles on heat transfer increased enhancement with increase in the Reynolds number. Sheikhzadeh et al. (2012) numerically investigated laminar mixed convection of $\mathrm{Cu}$-water nanofluid in two-sided lid-driven enclosures. The moving vertical walls of the enclosure were maintained at a constant temperature, while the horizontal walls were considered insulated. It was observed that the direction of the moving wall mainly affected the flow field, temperature gradient, and heat transfer. Mansour et al. (2010) presented a numerical investigation of laminar mixed-convection cooling of a heat source embedded on the bottom wall of an enclosure filled with nanofluids. The influence of governing parameters, such as the Rayleigh number, Reynolds number, location and geometry of the heat source, the type of nanofluid, and solid volume fraction of nanoparticles on the cooling performance was studied. They concluded that the Nusselt number was increased by adding titanium oxide $\left(\mathrm{TiO}_{2}\right)$ nanoparticles to the base fluid. Mansour and Ahmed (2013), using the finite volume method, numerically investigated mixed convection in double lid-driven enclosures with a heat source embedded in the left sidewall and with an $\mathrm{Al}_{2} \mathrm{O}_{3}$-water nanofluid. Four cases were considered depending on the direction of the movement of the walls. It was found that the fluid flow and heat transfer characteristics depended strongly on the direction of the movement of horizontal walls. Also, a significant heat transfer enhancement was obtained due to the presence of nanoparticles. Hussein et al. (2013) numerically investigated mixed convection in a rectangular inclined lid-driven cavity filled with water-based nanofluids. Since a uniform heat source was located on a part of the left inclined sidewall of the cavity, the right inclined sidewall was considered adiabatic together with the remaining parts of the left inclined sidewall. The top and bottom walls were maintained at a low temperature, and the top wall moved from left to right with a uniform lid-driven velocity. It was found that the local Nusselt number was decreased as the inclination angle and the solid volume fraction were increased. Esfe et al. (2013) numerically studied mixed convection within nanofluid-filled cavities with two adjacent moving walls by using the finite volume approach. It was found that by adding nanoparticles to the fluid, the strength of the vortices was increased. Also, they concluded that the average Nusselt number was increased by increasing the volume fraction of nanoparticles. Kalteh et al. (2014) using the finite-difference method, numerically studied steady laminar mixed-convection flow in a lid-driven square cavity with a triangular heat source and with a water-based nanofluid filling the cavity. The left and bottom walls of the enclosure were kept insulated, the top wall, which was moving at a constant speed, was maintained at a constant temperature, and the right wall was kept at a constant temperature also. It was found that suspending nanoparticles in a pure fluid lead to a significant increase in the heat transfer rate. Muthtamilselvan 
and Doh (2014) numerically investigated mixed convection in a lid-driven square cavity filled with a $\mathrm{Cu}$-water nanofluid in the presence of internal heat generation. The bottom wall was uniformly and nonuniformly heated, while two vertical walls were considered fixed and thermally insulated. The top wall was moving from left to right at a constant speed. The presented results showed that the solid volume fraction played a significant role in the flow and thermal fields, as well as the Nusselt number distributions for different flow configurations. Ferdows et al. (2012) numerically studied MHD mixed-convection boundary-layer flow of a nanofluid over an exponentially stretching sheet. The numerical solutions for the wall skin friction coefficient, the heat and mass transfer coefficients, as well as the velocity, temperature, and concentration profiles were computed and discussed graphically. Keshtkar et al. (2014) numerically investigated the effect of heat source/sink on MHD mixed-convection boundary-layer flow past a vertical permeable surface embedded in a porous medium saturated with a nanofluid. Three types of metallic or nonmetallic nanoparticles [i.e., copper $(\mathrm{Cu})$, alumina $\left(\mathrm{Al}_{2} \mathrm{O}_{3}\right)$, and titanium $\left(\mathrm{TiO}_{2}\right)$ ] were considered by using a water-based fluid to investigate the effect of the solid volume fraction of the nanofluid. It was found that the suction parameter increased the velocity profiles and delayed the separation of the boundary layer, while the injection parameter decreased the velocity profiles. Other useful researches conducted to simulate mixed-convection heat transfer using a nanofluid under different conditions can be found in Talebi et al. (2010), Alinia et al. (2011), Chamkha and Abu-Nada (2012), Arani et al. (2012), and Garoosi et al. (2014). Recently, much attention has been devoted to micropolar fluids due to their many practical applications such as liquid crystals, low-concentration suspension flow, blood rheology, the presence of dust or smoke, exotic lubricants, and the effect of dirt in a journal bearing. In fact, micropolar fluids are those with microstructure belonging to a complex class of non-Newtonian fluids with a nonsymmetrical stress tensor. Physically, they represent fluids consisting of random particles suspended in a viscous medium (Mahmoud and Waheed, 2010). The theory of the micropolar fluid was first suggested by Eringen (1965) to take into account the effects of the local structure and micromotions of the fluid particles, which cannot be described by the classical models. Anyway, some investigations consider mixed convention in an enclosure filled with a micropolar fluid such as Hsu and Wang (2000). A literature survey indicates that no studies at all have been done on the magnetohydrodynamic mixed convection in a lid-driven trapezoidal enclosure partially heated from its bottom wall and filled with a micropolar nanofluid. Most of the published papers are related to mixed-convection heat transfer in classical enclosures filled with Newtonian nanofluids. The extension to the non-Newtonian or micropolar nanofluids is not considered previously anywhere. To reach this goal, the objective of the present work is to examine the effects of Richardson number, Hartmann number, the type of nanofluid, length and location of the heat source, solid volume fraction, magnetic field orientation angle, and dimensionless viscosity on the magnetohydrody-

Volume 9, Issue 4, 2018 
namic mixed convection in a two-dimensional lid-driven trapezoidal enclosure filled with micropolar nanofluids.

\section{MATHEMATICAL MODELING}

\subsection{Governing Equations and Geometric Configuration}

Figure 1 shows a schematic diagram of a two-dimensional trapezoidal enclosure of height $H$ and width $w$ along with the important geometric parameters. The fluid inside the enclosure is represented by micropolar water-based nanofluids containing four different types of solid spherical nanoparticles (i.e., $\mathrm{Cu}, \mathrm{Ag}, \mathrm{Al}_{2} \mathrm{O}_{3}$, and $\mathrm{TiO}_{2}$ ). The nanofluid is assumed to be incompressible and the flow is assumed to be twodimensional, laminar, and steady. It is assumed that the base fluid (i.e., water) and nanoparticles are in a thermal equilibrium state. The Reynolds number is considered fixed during the calculation at $\operatorname{Re}=10$. The solid volume fractions $\phi$ varied as $0 \leq \phi \leq 0.02$, while the dimensionless length of the heat source $B$ varied as $0.1 \leq B \leq 0.8$. The dimensionless location of the heat source $D$ varied as $0.3 \leq D \leq 0.7$, while the dimensionless viscosity $K$ varied as $0 \leq K \leq 5$, respectively. The range of the Richardson number is taken as $0.04 \leq \mathrm{Ri} \leq 100$, while the range of the Hartmann number was taken to be $0 \leq \mathrm{Ha} \leq 50$, respectively. The thermophysical properties of the base fluid and nanoparticles are tabulated in Table 1. It is assumed that the upper lid is moving from left to right with a constant velocity $U_{0}$. The right and left inclined vertical walls are kept at a low temperature, while the top lid wall is assumed to be thermally insulated. In order to create the buoyancy effect, a uniform heat source with a constant volumetric rate $q$ " is located in a part of the

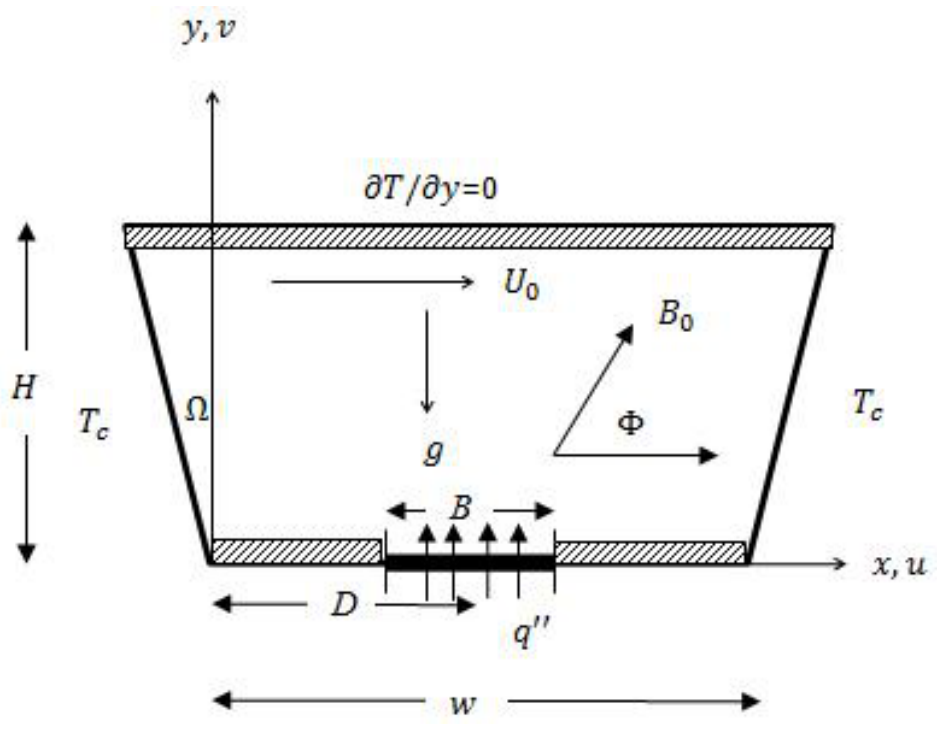

FIG. 1: Physical model and the coordinates system 
TABLE 1: Thermophysical properties of water and nanoparticles

\begin{tabular}{|c|c|c|c|c|c|}
\hline Property & Water & Copper (Cu) & Silver $\mathbf{( A g )}$ & $\begin{array}{c}\text { Alumina } \\
\mathbf{( A l}_{\left.\mathbf{2} \mathbf{O}_{3}\right)}\end{array}$ & $\begin{array}{c}\text { Titanium Dioxide } \\
\mathbf{( T i O}_{\mathbf{2}} \mathbf{)}\end{array}$ \\
\hline$\rho$ & 997.1 & 8933 & 10.500 & 3970 & 4250 \\
\hline$c_{p}$ & 4179 & 385 & 235 & 765 & 686.2 \\
\hline$k$ & 0.613 & 401 & 429 & 40 & 8.9538 \\
\hline$\beta$ & $21 \times 10^{-5}$ & $1.67 \times 10^{-5}$ & $1.89 \times 10^{-5}$ & $0.85 \times 10^{-5}$ & $0.9 \times 10^{-5}$ \\
\hline$\sigma$ & 0.05 & $5.96 \times 10^{7}$ & $3.6 \times 10^{7}$ & $1 \times 10^{-10}$ & $1 \times 10^{-12}$ \\
\hline
\end{tabular}

bottom wall, while the remaining parts of the same wall are considered adiabatic. The flow in the trapezoidal enclosure is subjected to a uniform magnetic field $B_{0}$ in the range of the orientation angles $0^{\circ} \leq \Phi \leq 180^{\circ}$. The thermophysical properties of both the base fluid and nanofluids are assumed to be constant except for the density variation, which is modeled using the Boussinesq model. Under these assumptions, the dimensional governing continuity, momentum, angular momentum, and energy equations are, respectively, expressed as [see (Eringen, 1965; Ahmadi, 1976)]

$$
\begin{gathered}
\frac{\partial u}{\partial x}+\frac{\partial v}{\partial y}=0 \\
u \frac{\partial u}{\partial x}+v \frac{\partial u}{\partial y}=\frac{1}{\rho_{\mathrm{nf}}} \frac{\partial p}{\partial x}+\frac{1}{\rho_{\mathrm{nf}}}\left(\mu_{\mathrm{nf}}+k\right)\left(\frac{\partial^{2} u}{\partial x^{2}}+\frac{\partial^{2} u}{\partial y^{2}}\right) \\
+\frac{k}{\rho_{\mathrm{nf}}} \frac{\partial n}{\partial y}+\frac{\sigma_{\mathrm{nf}} B_{0}^{2}}{\rho_{\mathrm{nf}}}\left(v \sin \Phi \cos \Phi-u \sin ^{2} \Phi\right) \\
u \frac{\partial v}{\partial x}+v \frac{\partial v}{\partial y}=-\frac{1}{\rho_{\mathrm{nf}}} \frac{\partial p}{\partial y}+\frac{1}{\rho_{\mathrm{nf}}}\left(\mu_{\mathrm{nf}}+k\right)\left(\frac{\partial^{2} v}{\partial x^{2}}+\frac{\partial^{2} v}{\partial y^{2}}\right) \\
\frac{k n}{\rho_{\mathrm{nf}}} \frac{\partial x}{\partial x} \frac{\sigma_{\mathrm{nf}} B_{0}^{2}}{\rho_{\mathrm{nf}}}\left(u \sin \Phi \cos \Phi-v \cos ^{2} \Phi\right)+\frac{(\rho \beta)_{\mathrm{nf}}}{\rho_{\mathrm{nf}}} g\left(T-T_{c}\right) \\
u \frac{\partial n}{\partial x}+v \frac{\partial n}{\partial y}=\frac{\gamma_{\mathrm{nf}}}{\rho_{\mathrm{nf}} j}\left(\frac{\partial^{2} n}{\partial x^{2}}+\frac{\partial^{2} n}{\partial y^{2}}\right)-\frac{2 k}{\rho_{\mathrm{nf}} j}+\frac{k}{\rho_{\mathrm{nf}} j}\left(\frac{\partial v}{\partial x}-\frac{\partial u}{\partial y}\right) \\
u \frac{\partial T}{\partial x}+v \frac{\partial T}{\partial y}=\alpha_{\mathrm{nf}}\left(\frac{\partial^{2} T}{\partial x^{2}}+\frac{\partial^{2} T}{\partial y^{2}}\right)
\end{gathered}
$$


In the present study, the relations which depend only on the nanoparticles volume fraction are adopted. These relations are used in many previous studies (Rees and Pop, 1998; Khanafer et al., 2003; Oztop and Abu-Nada, 2008) and can be written as follows:

$$
\gamma_{\mathrm{nf}}=\left(\mu_{\mathrm{nf}}+\frac{k}{2}\right) j .
$$

The effective density, heat capacity, thermal expansion coefficient, and the thermal diffusivity of the nanofluid are respectively given by

$$
\begin{gathered}
\rho_{\mathrm{nf}}=(1-\phi) \rho_{\mathrm{f}}+\phi \rho_{\mathrm{p}}, \\
\left(\rho c_{\mathrm{p}}\right)_{\mathrm{nf}}=(1-\phi)\left(\rho c_{\mathrm{p}}\right)_{\mathrm{f}}+\phi\left(\rho c_{\mathrm{p}}\right)_{\mathrm{p}}, \\
(\rho \beta)_{\mathrm{nf}}=(1-\phi)(\rho \beta)_{\mathrm{f}}+\phi(\rho \beta)_{\mathrm{p}}, \\
\alpha_{\mathrm{nf}}=\frac{k_{\mathrm{nf}}}{\left(\rho c_{\mathrm{p}}\right)_{\mathrm{nf}}} .
\end{gathered}
$$

The thermal conductivity of the nanofluid is given by (Aminossadati and Ghasemi, 2009; Godson et al., 2010; Pak and Cho, 1998; Basak et al., 2009)

$$
\begin{gathered}
\frac{k_{\mathrm{nf}}}{k_{\mathrm{f}}}=\frac{\left(k_{\mathrm{p}}+2 k_{\mathrm{f}}+2 \phi\left(k_{\mathrm{p}}-k_{\mathrm{f}}\right)(1+R)^{3}\right.}{\left(k_{\mathrm{p}}+2 k_{\mathrm{f}}-2 \phi\left(k_{\mathrm{p}}-k_{\mathrm{f}}\right)(1+R)^{3}\right.}, \\
\text { where } R=0.1[\text { for } \mathrm{Cu} \text { nanoparticles] }, \\
\frac{k_{\mathrm{nf}}}{k_{\mathrm{f}}}=(0.9508+0.9692 \phi)[\text { for Ag nanoparticles] } \\
\frac{k_{\mathrm{nf}}}{k_{\mathrm{f}}}=(1+7.47 \phi)\left[\text { for } \mathrm{Al}_{2} \mathrm{O}_{3}\right. \text { nanoparticles] } \\
\frac{k_{\mathrm{nf}}}{k_{\mathrm{f}}}=\left(1+2.92 \phi-11.99 \phi^{2}\right)\left[\text { for } \mathrm{TiO}_{2} \text { nanoparticles] },\right.
\end{gathered}
$$

and the effective dynamic viscosity of the nanofluid is given by (Aminossadati and Ghasemi, 2009; Godson et al., 2010; Pak and Cho, 1998) 


$$
\begin{gathered}
\mu_{\mathrm{nf}}=\frac{\mu_{\mathrm{f}}}{(1-\phi)^{2.5}} \text { [for Cu nanoparticles] } \\
\mu_{\mathrm{nf}}=\mu_{\mathrm{f}}\left(1.005+0.49 \phi-0.1149 \phi^{2}\right) \text { [for Ag nanoparticles] } \\
\left.\mu_{\mathrm{nf}}=\mu_{\mathrm{f}}\left(1+39.11 \phi+533.9 \phi^{2}\right) \text { [for } \mathrm{Al}_{2} \mathrm{O}_{3} \text { nanoparticles }\right] \\
\mu_{\mathrm{nf}}=\mu_{\mathrm{f}}\left(1+5.45 \phi+108.2 \phi^{2}\right) \text { [for } \mathrm{TiO}_{2} \text { nanoparticles] }
\end{gathered}
$$

Moreover, the electrical conductivity of the nanofluid is given by

$$
\frac{\sigma_{\mathrm{nf}}}{\sigma_{\mathrm{f}}}=1+\frac{3\left(\frac{\sigma_{\mathrm{p}}}{\sigma_{\mathrm{f}}}-1\right) \phi}{\left(\frac{\sigma_{\mathrm{p}}}{\sigma_{\mathrm{f}}}+2\right)-\left(\frac{\sigma_{\mathrm{p}}}{\sigma_{\mathrm{f}}}-1\right) \phi} .
$$

By introducing the following dimensionless variables as listed below

$$
\begin{gathered}
X=\frac{x}{w}, \quad Y=\frac{y}{H}, \quad U=\frac{u}{U_{0}}, \quad V=\frac{v}{U_{0}}, \\
P=\frac{p}{\rho_{\mathrm{nf}} U_{0}^{2}}, \quad \theta=\frac{\left(T-T_{\mathrm{c}}\right)}{H \times q^{\prime \prime}} k_{\mathrm{f}}, \quad N=\frac{n \times H}{U_{0}}, \\
\chi=\frac{H^{2}}{j}, \quad K=\frac{k}{\mu_{\mathrm{f}}}, \quad \operatorname{Pr}=\frac{v_{\mathrm{f}}}{\alpha_{\mathrm{f}}}, \\
\operatorname{Ri}=\frac{\operatorname{Ra}}{\operatorname{Pr}_{\operatorname{Re}}^{2}}, \quad H a=B_{0} H \sqrt{\sigma_{\mathrm{f}} / \mu_{\mathrm{f}}}, \\
\operatorname{Ra}=\frac{g \beta_{\mathrm{f}} q^{\prime \prime} H^{4}}{\nu_{\mathrm{f}} \alpha_{\mathrm{f}} k_{\mathrm{f}}}, \quad \text { and } \quad \operatorname{Re}=\frac{U_{0} H}{\nu_{\mathrm{f}}},
\end{gathered}
$$

the dimensional equations (1)-(5) can be written in dimensionless form as follows:

$$
\frac{\partial U}{\partial X}+\frac{\partial V}{\partial Y}=0
$$




$$
\begin{aligned}
& U \frac{\partial U}{\partial X}+V \frac{\partial U}{\partial Y}=-\frac{\partial P}{\partial X}+\frac{1}{\operatorname{Re}}\left(\frac{\rho_{\mathrm{f}}}{\rho_{\mathrm{nf}}}\right)\left(\frac{\mu_{\mathrm{nf}}}{\mu_{\mathrm{f}}}+K\right)\left(\frac{\partial^{2} U}{\partial X^{2}}+\frac{\partial^{2} U}{\partial Y^{2}}\right) \\
& +\frac{K}{\operatorname{Re}}\left(\frac{\rho_{\mathrm{f}}}{\rho_{\mathrm{nf}}}\right) \frac{\partial N}{\partial Y}+\frac{\mathrm{Ha}^{2}}{\operatorname{Re}}\left(\frac{\rho_{\mathrm{f}}}{\rho_{\mathrm{nf}}}\right)\left(\frac{\sigma_{\mathrm{nf}}}{\sigma_{\mathrm{f}}}\right)\left(V \sin \Phi \cos \Phi-u \sin ^{2} \Phi\right), \\
& U \frac{\partial V}{\partial X}+V \frac{\partial V}{\partial Y}=-\frac{\partial P}{\partial Y}+\frac{1}{\operatorname{Re}}\left(\frac{\rho_{\mathrm{f}}}{\rho_{\mathrm{nf}}}\right)\left(\frac{\mu_{\mathrm{nf}}}{\mu_{\mathrm{f}}}+K\right)\left(\frac{\partial^{2} V}{\partial X^{2}}+\frac{\partial^{2} V}{\partial Y^{2}}\right)-\frac{K}{\operatorname{Re}}\left(\frac{\rho_{\mathrm{f}}}{\rho_{\mathrm{nf}}}\right) \frac{\partial N}{\partial X} \\
& +\frac{\mathrm{Ha}^{2}}{\operatorname{Re}}\left(\frac{\rho_{\mathrm{f}}}{\rho_{\mathrm{nf}}}\right)\left(\frac{\sigma_{\mathrm{nf}}}{\sigma_{f}}\right)\left(U \sin \Phi \cos \Phi-v \cos ^{2} \Phi\right)+\mathrm{Ri}\left(\frac{\rho_{\mathrm{f}}}{\rho_{\mathrm{nf}}}\right)\left(\frac{(\rho \beta)_{\mathrm{nf}}}{\rho_{\mathrm{f}} \beta_{\mathrm{f}}}\right) \theta, \\
& U \frac{\partial N}{\partial X}+V \frac{\partial N}{\partial Y}=\frac{1}{\operatorname{Re}}\left(\frac{\rho_{\mathrm{f}}}{\rho_{\mathrm{nf}}}\right)\left(\frac{\mu_{\mathrm{nf}}}{\mu_{\mathrm{f}}}+\frac{K}{2}\right)\left(\frac{\partial^{2} N}{\partial X^{2}}+\frac{\partial^{2} N}{\partial Y^{2}}\right) \\
& -\frac{K}{\operatorname{Re}} \chi\left(\frac{\rho_{\mathrm{f}}}{\rho_{\mathrm{nf}}}\right)\left(2 N-\left(\frac{\partial V}{\partial X}-\frac{\partial U}{\partial Y}\right)\right) \\
& U \frac{\partial \theta}{\partial X}+V \frac{\partial \theta}{\partial Y}=\left(\frac{1}{\operatorname{Pr} \operatorname{Re}}\right) \frac{\alpha_{\mathrm{nf}}}{\alpha_{\mathrm{f}}}\left(\frac{\partial^{2} \theta}{\partial X^{2}}+\frac{\partial^{2} \theta}{\partial Y^{2}}\right)
\end{aligned}
$$

\subsection{Boundary Conditions}

The dimensionless form of the boundary conditions for Eqs. (21)-(25) is expressed as follows:

left inclined sidewall:

$$
X+Y \tan \Omega=0, \quad 0 \leq Y \leq 1: U=V=\theta=0,
$$

right inclined sidewall:

$$
X-Y \tan \Omega=1, \quad 0 \leq Y \leq 1: U=V=\theta=0,
$$

top wall:

$$
Y=1,-\tan \Omega \leq V \leq 1+\tan \Omega: U=1, \quad V=0, \frac{\partial \theta}{\partial Y}=0,
$$

bottom wall:

$$
Y=0,0 \leq X \leq 1: U=V=0,
$$




$$
\begin{gathered}
\frac{\partial \theta}{\partial Y}=-\frac{k_{\mathrm{f}}}{k_{\mathrm{nf}}}, \quad(D-0.5 B) \leq X \leq(D+0.5 B) \\
\frac{\partial \theta}{\partial Y}=0, \text { otherwise } \\
N=-\lambda \frac{\partial \theta}{\partial n^{*}}
\end{gathered}
$$

where $n^{*}$ is the unit vector normal to the wall.

\subsection{Local and Average Nusselt Numbers}

The local Nusselt number along the heat source surface can be written as

$$
\mathrm{Nu}_{s}=-\left(\frac{\partial \theta}{\partial Y}\right)_{Y=0},
$$

while the average Nusselt number is given by

$$
\mathrm{Nu}_{m}=\int_{0}^{1} \mathrm{Nu}_{s} d X .
$$

\section{NUMERICAL METHOD AND VERIFICATION}

The dimensionless governing equations (21)-(25) with dimensionless boundary conditions (26) are solved numerically using the finite difference method. Firstly, the pressure terms in Eqs. (22) and (23) are eliminated by the differentiation equation (22) with respect to $Y$ and Eq. (23) with respect to $X$ and subtract Eq. (22) from Eq. (23). Then, these equations are transformed to the vorticity-stream function forms. Secondly, the first and second derivatives in the resulting system are approximated using central difference quotients. The resulting system of discretized equations was solved iteratively. As convergence criteria, $10^{-6}$ is chosen for all dependent variables, and the value of 0.1 is used for the underrelaxation parameter. The number of grid points is taken as $129 \times 61$ with uniform spaced mesh in both $X$ and $Y$ directions. Further verification is performed by using the present numerical algorithm to simulate the same problem considered by Basak et al. (2009) using the same geometry and boundary conditions for laminar natural-convection flow in a trapezoidal enclosure filled with porous medium. The comparison for both streamlines and isotherms is given in Fig. 2. Very good agreement is found between the present results and those obtained by Basak et al. (2009). Therefore 


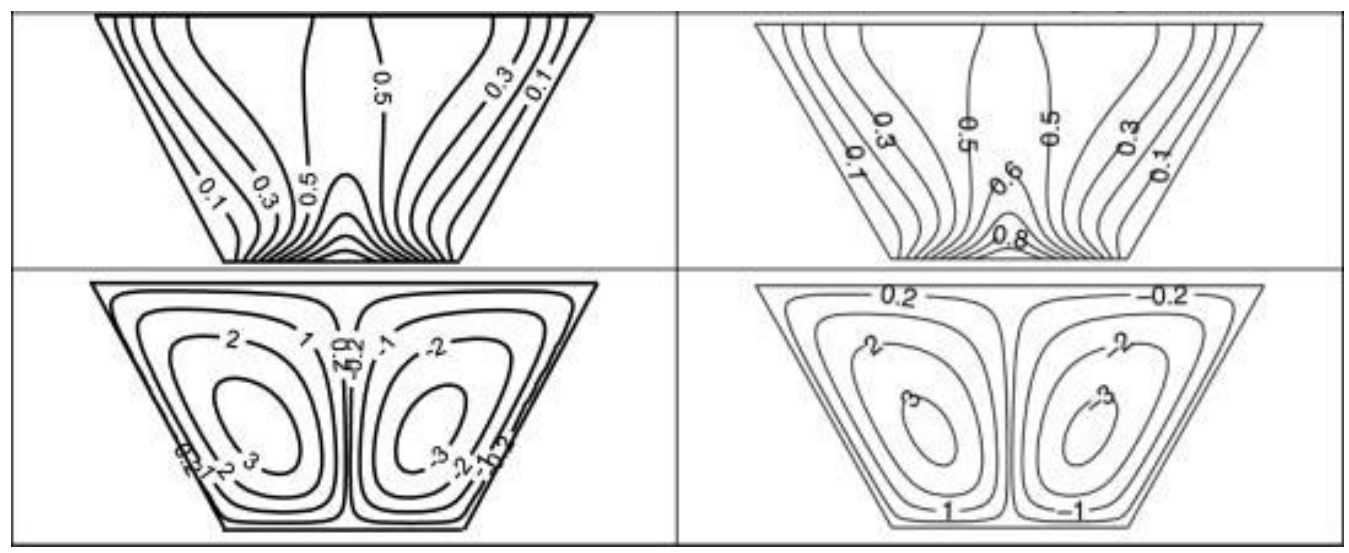

FIG. 2: Comparison of isotherms (top) and streamlines (bottom) at $K=0, \phi=0$, and $\mathrm{Ha}=0$

the computational procedure is practicable and can predict mixed convection in a trapezoidal enclosure filled with a micropolar $\mathrm{Cu}$-water nanofluid in the presence of an inclined magnetic field.

\section{RESULTS AND DISCUSSION}

The laminar magnetohydrodynamic mixed-convection heat transfer of water-based micropolar nanofluids in a trapezoidal lid-driven enclosure with a uniform heat source located on a part of its bottom wall has been investigated numerically in this study. The results of the present study are shown in Figs. 3-19. The fluid in-

Natural convection

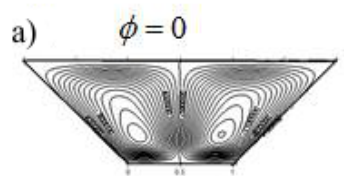

b)

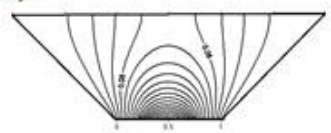

c)

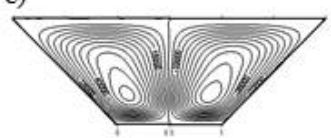

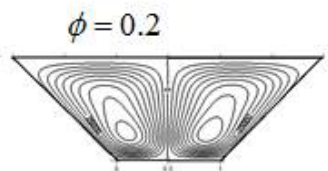
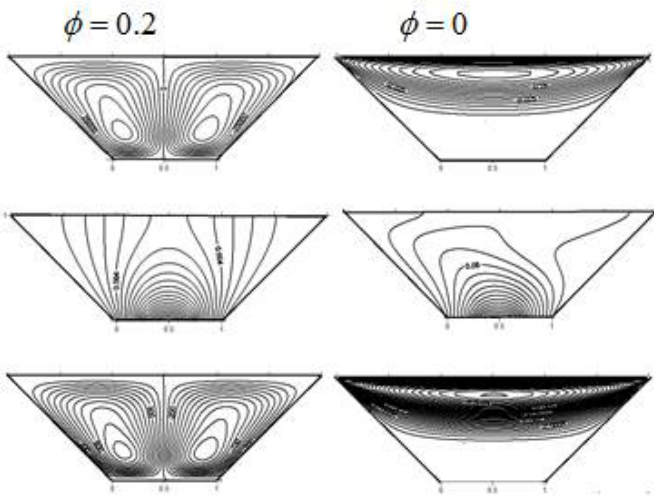

Forced convection
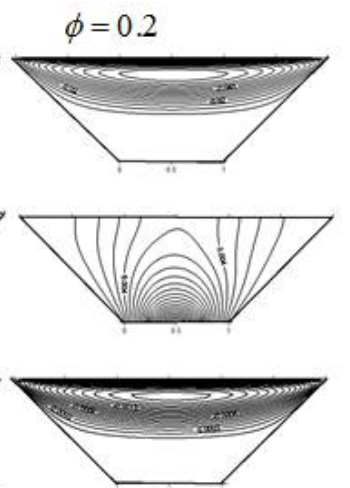

FIG. 3: Contours of streamlines (a), isotherms (b), and isolines of microrotation (c) for pure water $(\phi=0)$ and a micropolar $\mathrm{Cu}$-water nanofluid $(\phi=0.2)$ at $\Phi=0^{\circ}, K=0.5$, $D=0.5, B=0.5$, and $\mathrm{Ha}=0$ when $\mathrm{Re}=10$ for forced convection 


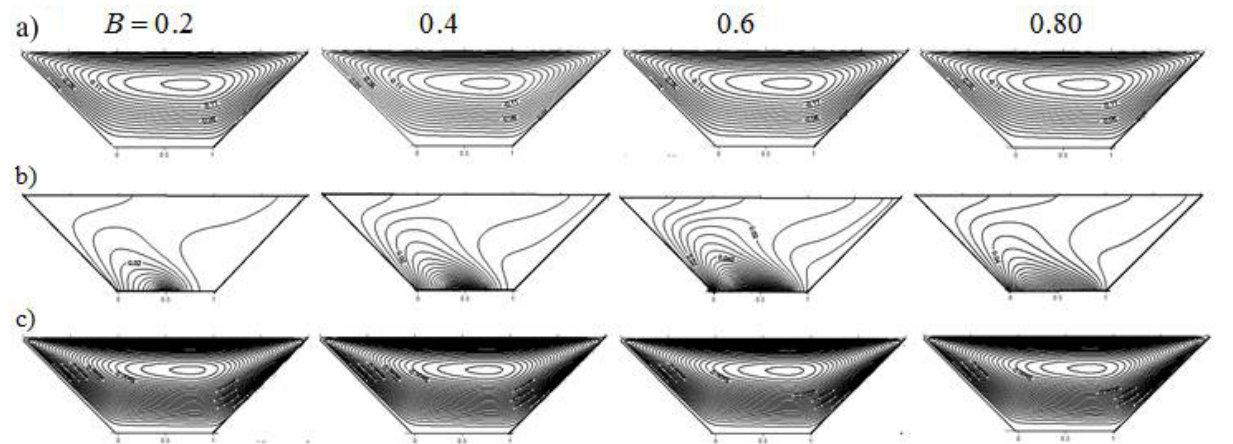

FIG. 4: Contours of streamlines (a), isotherms (b), and isolines of microrotation (c) of a micropolar $\mathrm{Cu}$-water nanofluid for various heat source lengths at $\mathrm{Ri}=1, \phi=0.05, \Phi=0^{\circ}$, $K=0.5, D=0.5$, and $\mathrm{Ha}=0$

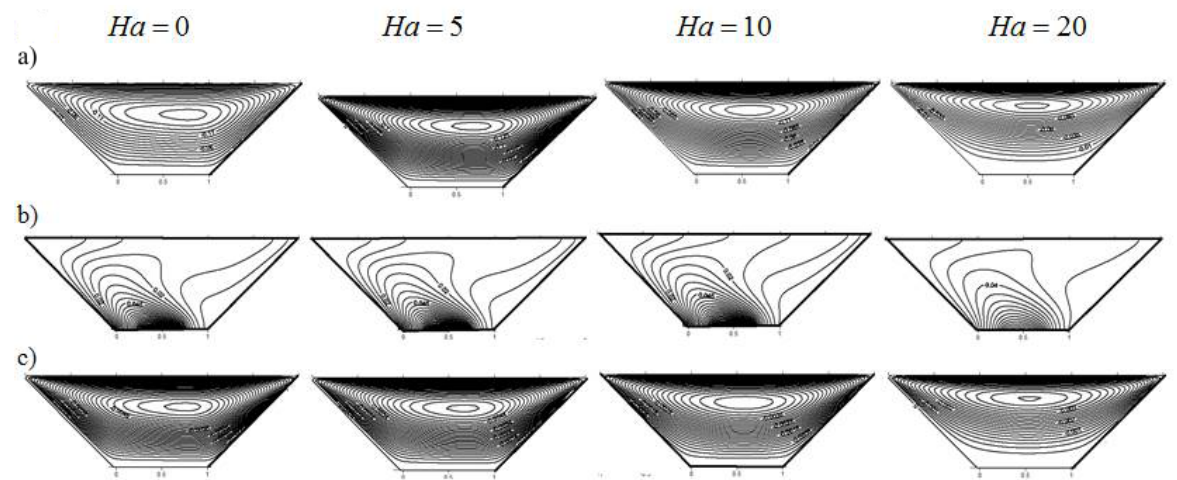

FIG. 5: Contours of streamlines (a), isotherms (b) and isolines of microrotation(c) of a micropolar $\mathrm{Cu}$-water nanofluid for various Hartmann numbers $\mathrm{Ha}$ at $\mathrm{Ri}=1, \phi=0.05$, $\Phi=0^{\circ}, K=0.5, D=0.5$, and $B=0.5$

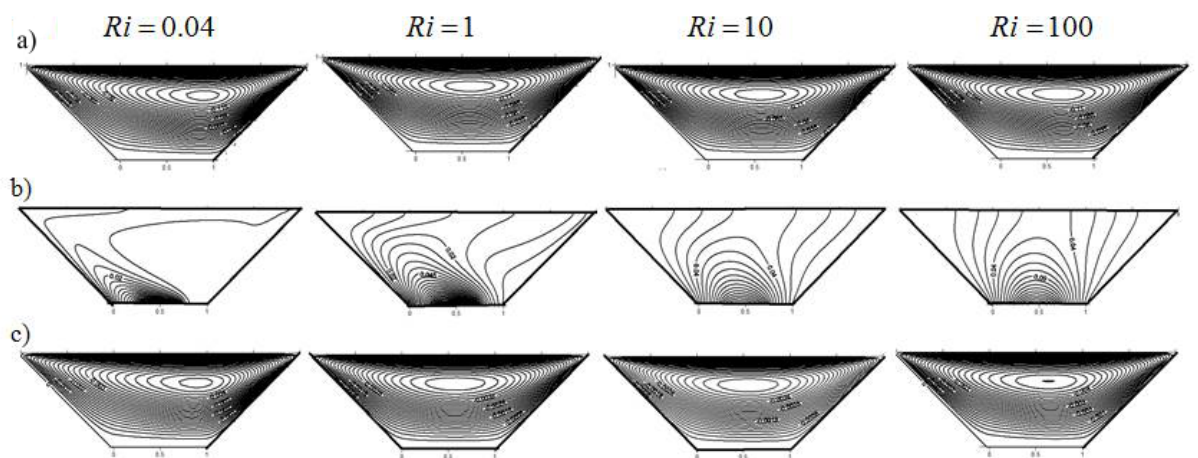

FIG. 6: Contours of streamlines (a), isotherms (b), and isolines of microrotation (c) of a $\mathrm{Cu}$-water micropolar nanofluid for various Richardson numbers $\mathrm{Ri}$ at $\mathrm{Ha}=10, \phi=0.05$, $\Phi=0^{\circ}, K=0.5, D=0.5$, and $B=0.5$ 


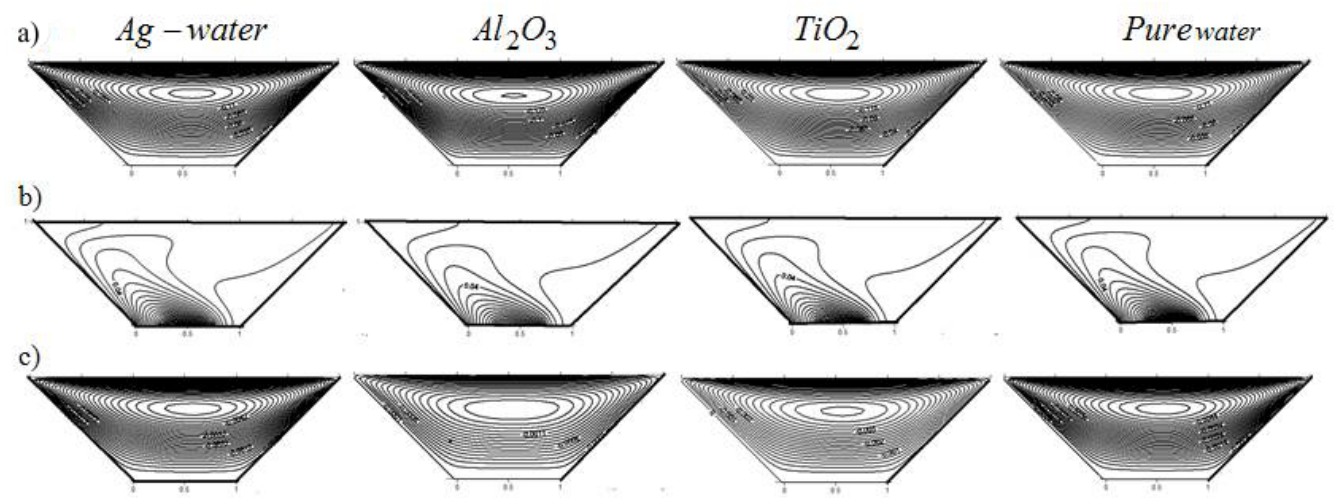

FIG. 7: Contours of streamlines (a), isotherms (b), and isolines of microrotation (c) for different nanofluids and pure water at $\mathrm{Ha}=10, \mathrm{Ri}=1, \phi=0.05, \Phi=0^{\circ}, K=0.5, D=0.5$, and $B=0.5$

side the enclosure is a water-based micropolar nanofluid containing four various types of solid spherical nanoparticles which are $\mathrm{Cu}, \mathrm{Ag}, \mathrm{Al}_{2} \mathrm{O}_{3}$, and $\mathrm{TiO}_{2}$. The results are presented in this study for the following fixed parameters: $\operatorname{Ra}=10^{3}, \chi=0.1$, $\lambda=0, \Omega=\pi / 4$.

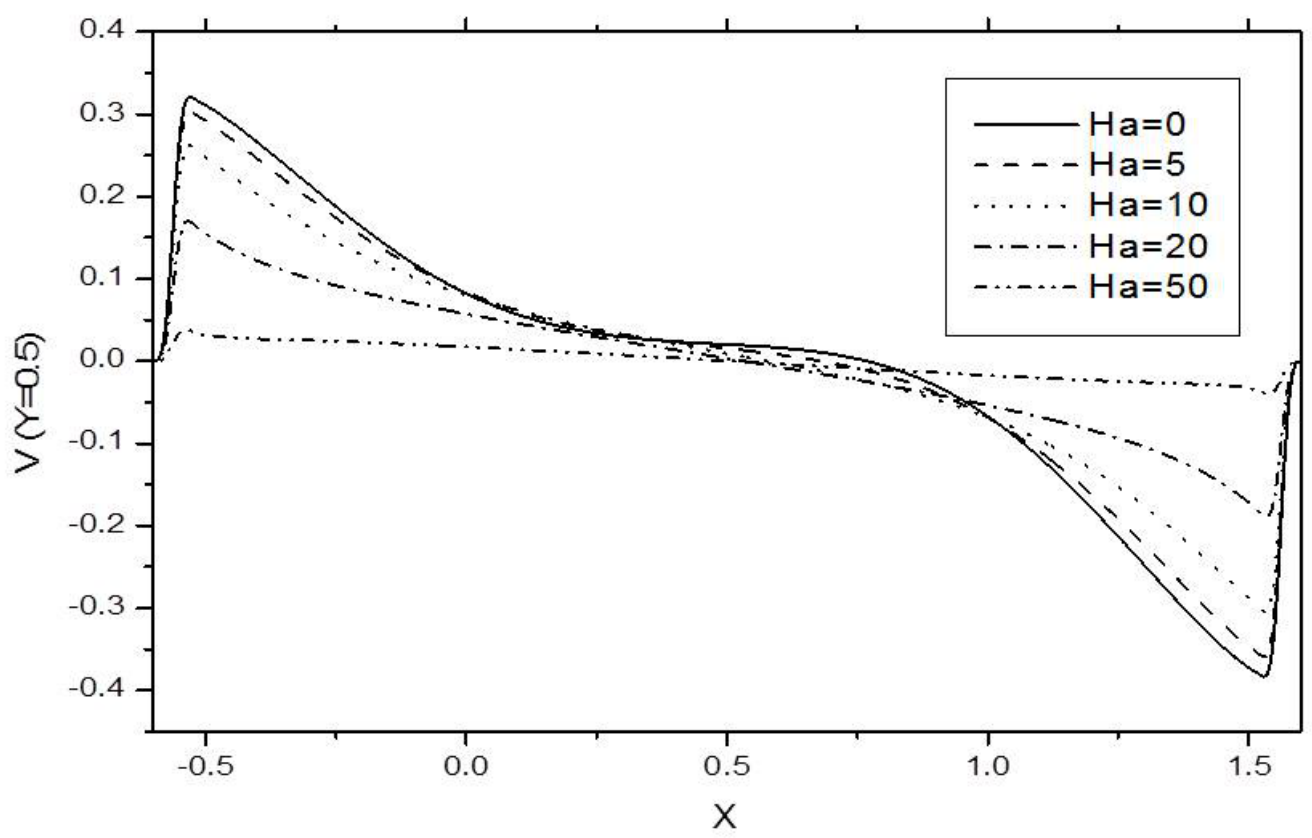

FIG. 8: Profiles of the vertical velocity component at the enclosure midsection for a micropolar $\mathrm{Cu}$-water nanofluid at $B=0.5, \mathrm{Ri}=1, D=0.5, \Phi=0^{\circ}, K=0.5$, and $\phi=0.05$ 


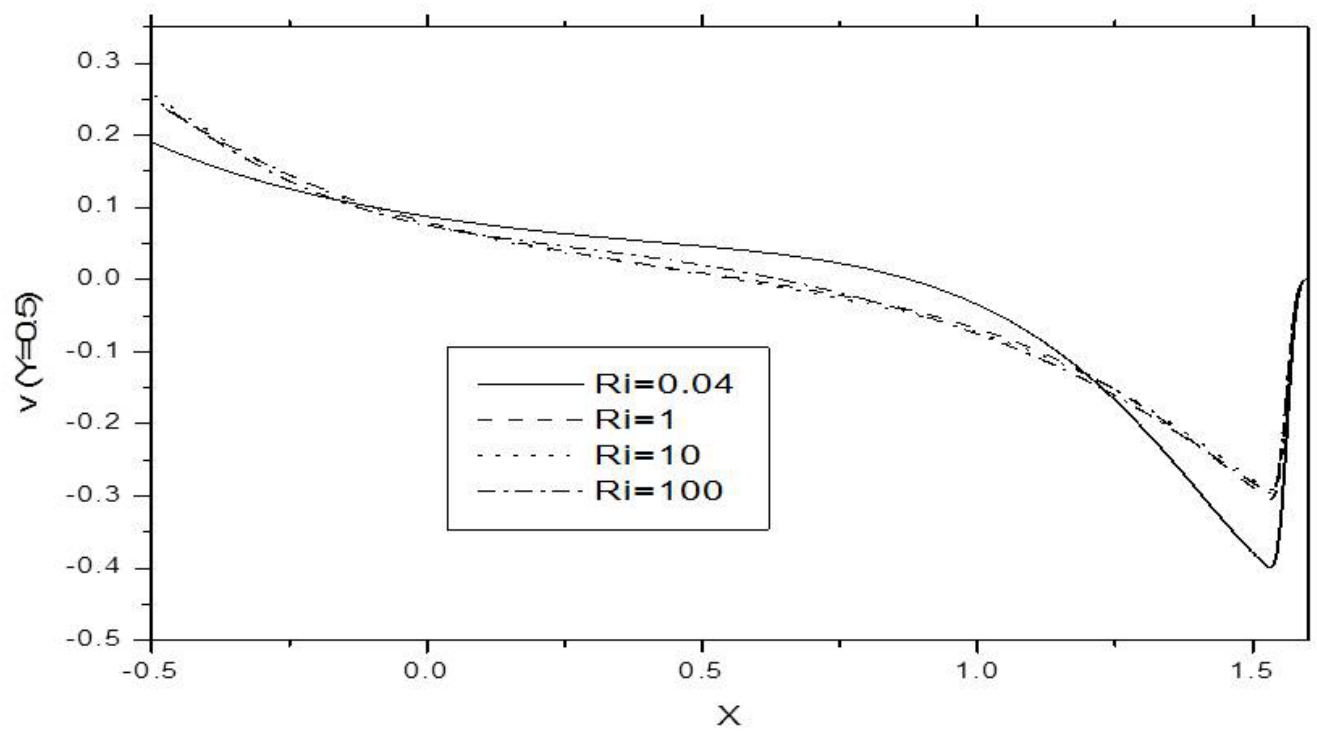

FIG. 9: Profiles of the vertical velocity component at the enclosure midsection for a micropolar Cu-water nanofluid at $B=0.5$, $\mathrm{Ha}=10, D=0.5, \Phi=0^{\circ}, K=0.5$, and $\phi=0.05$

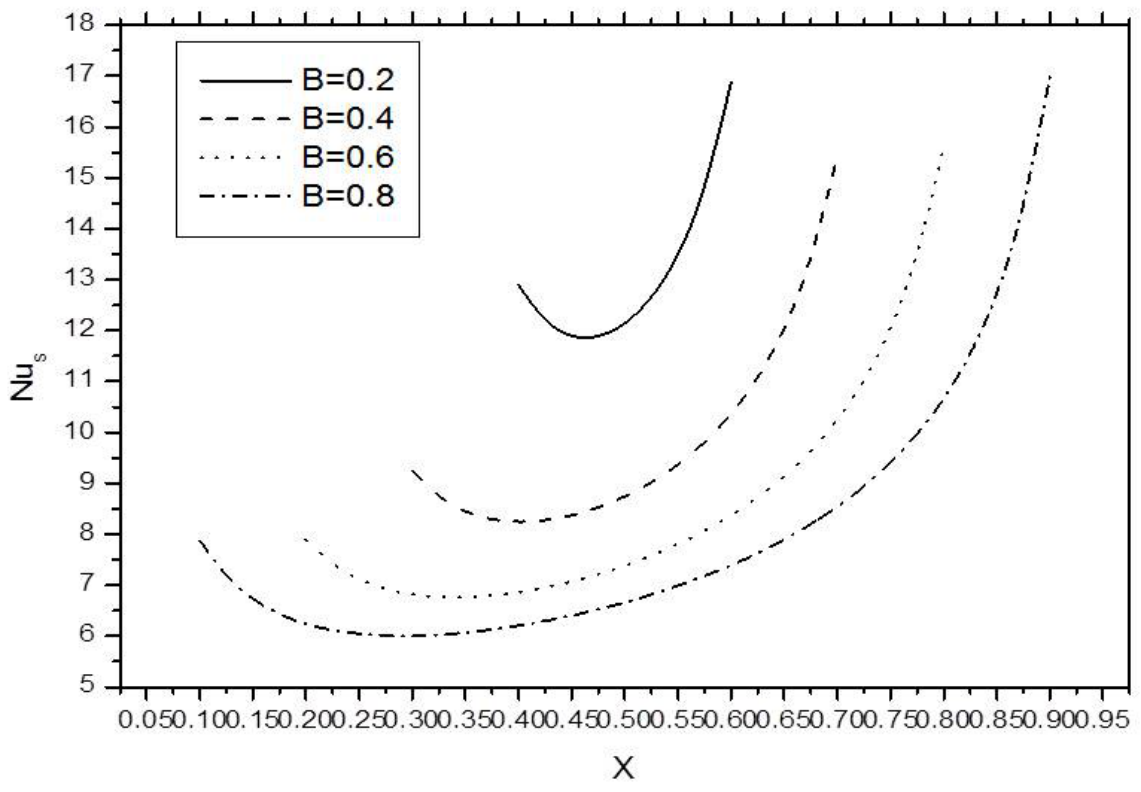

FIG. 10: Profiles of the local Nusselt number along the heat source for a micropolar $\mathrm{Cu}$-water nanofluid with different values of dimensionless length of the heat source $B$ at $D=0.5, K=0.5, \mathrm{Ri}=1, \phi=0.05, \Phi=0^{\circ}$, and $\mathrm{Ha}=0$ 


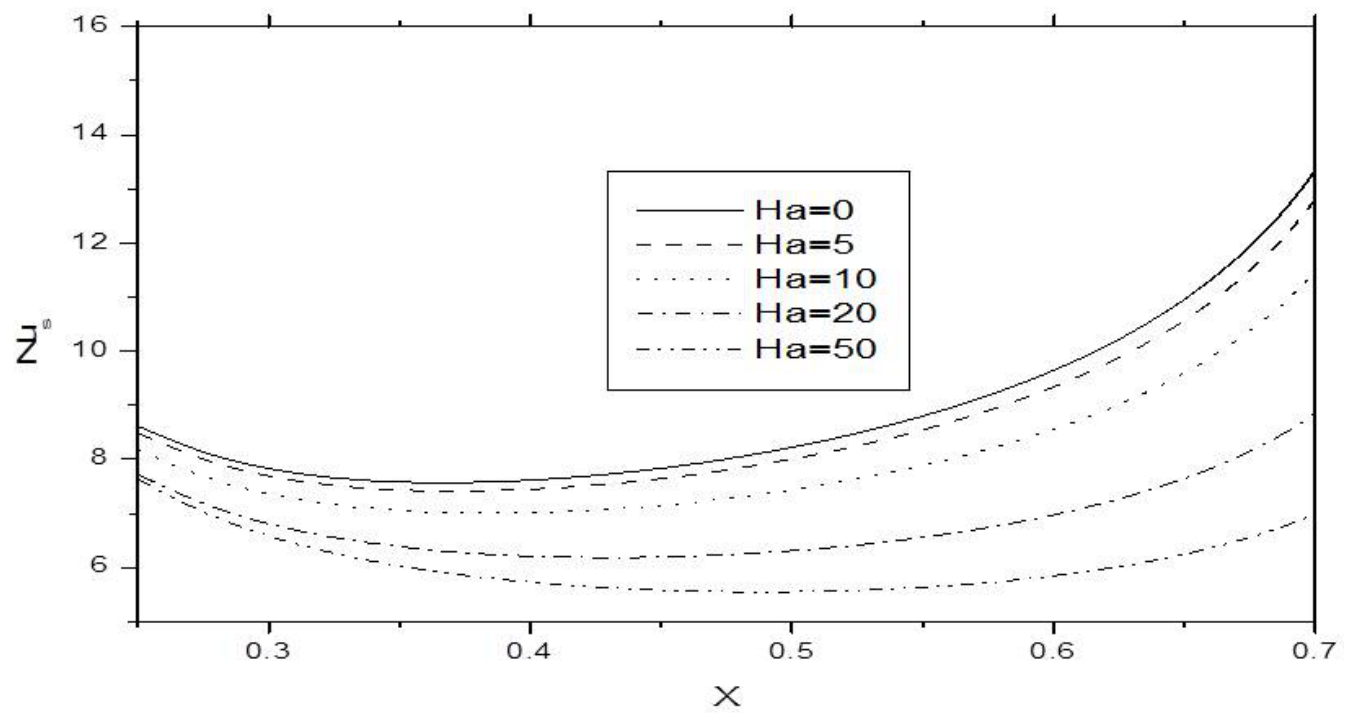

FIG. 11: Profiles of the local Nusselt number along the heat source for a micropolar $\mathrm{Cu}$-water nanofluid with different values of the Hartmann number $\mathrm{Ha}$ at $D=0.5, K=0.5$, $\mathrm{Ri}=1, \phi=0.05, \Phi=0^{\circ}$, and $B=0.5$

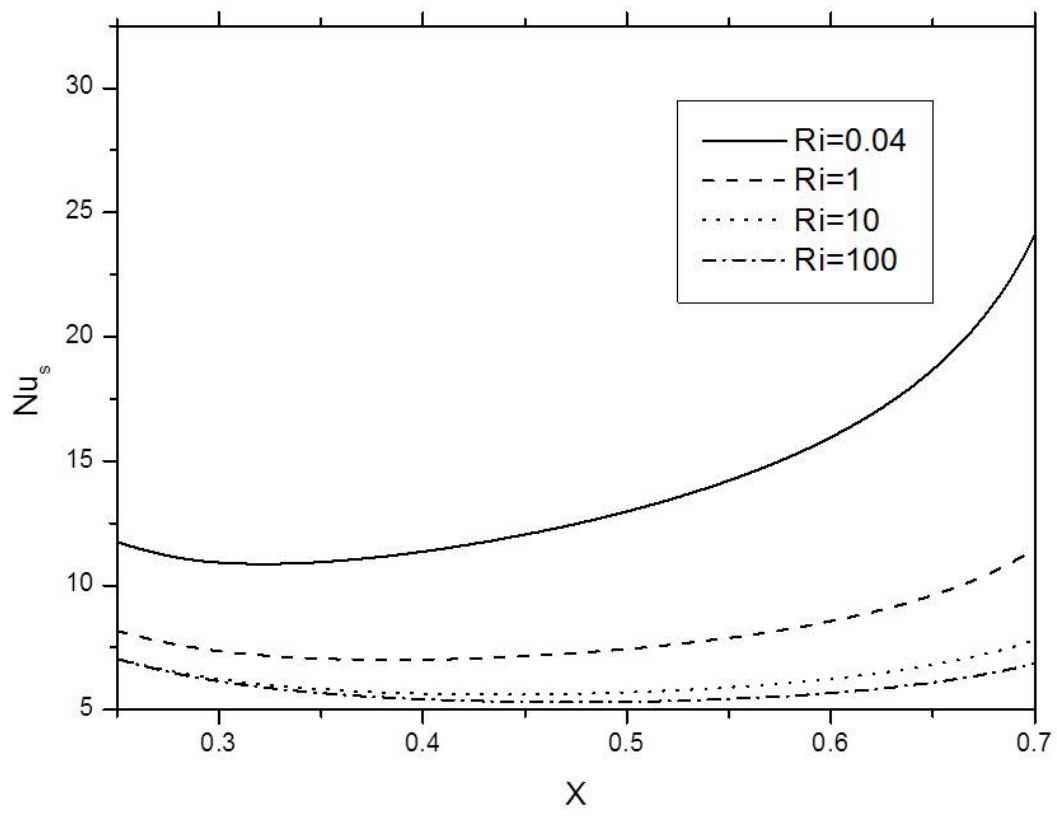

FIG. 12: Profiles of the local Nusselt number along the heat source for a micropolar $\mathrm{Cu}$-water nanofluid with different values of the Richardson number Ri at $D=0.5, K=0.5$, $\mathrm{Ha}=10, \phi=0.05, \Phi=0^{\circ}$, and $B=0.5$ 


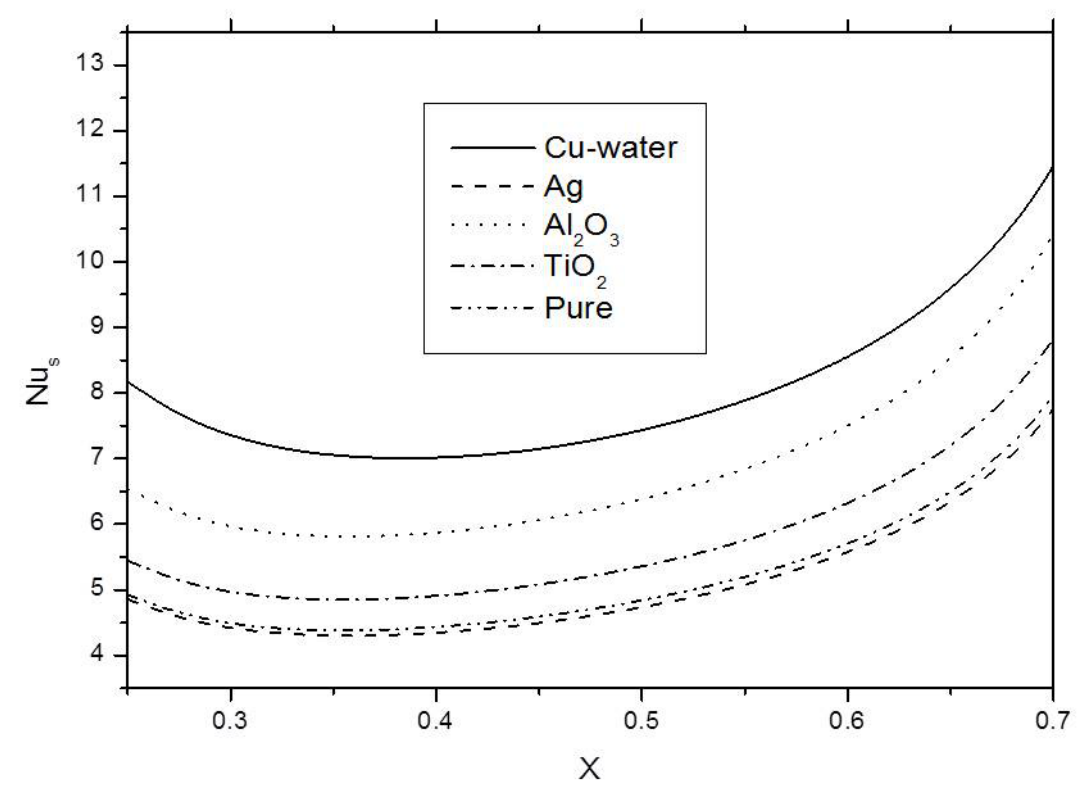

FIG. 13: Profiles of the local Nusselt number along the heat source for different nanofluids and pure water at $D=0.5, K=0.5, \mathrm{Ha}=10, \mathrm{Ri}=1, \phi=0.05, \Phi=0^{\circ}$, and $B=0.5$

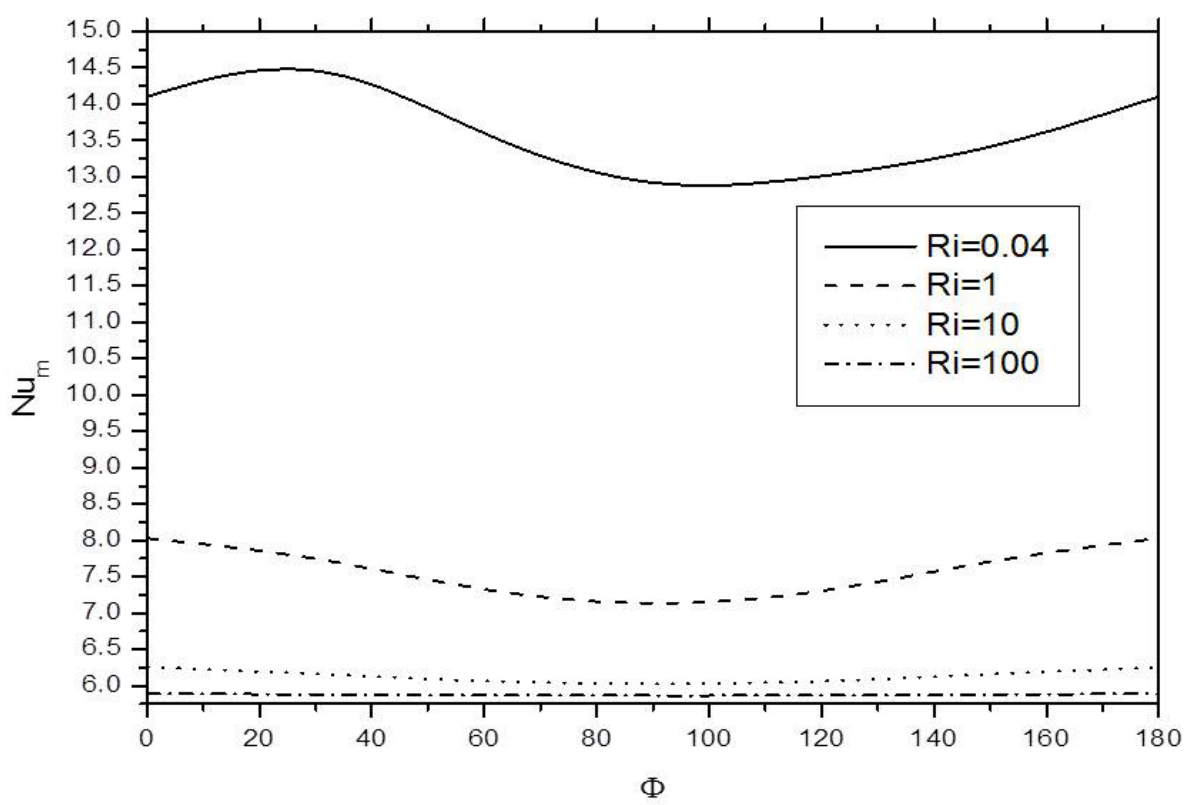

FIG. 14: Profiles of the average Nusselt number for a micropolar $\mathrm{Cu}$-water nanofluid with different values of the magnetic field orientation angle and the Richardson number $\mathrm{Ri}$ at $D=0.5, K=0.5, \mathrm{Ha}=10, \phi=0.05$, and $B=0.5$ 


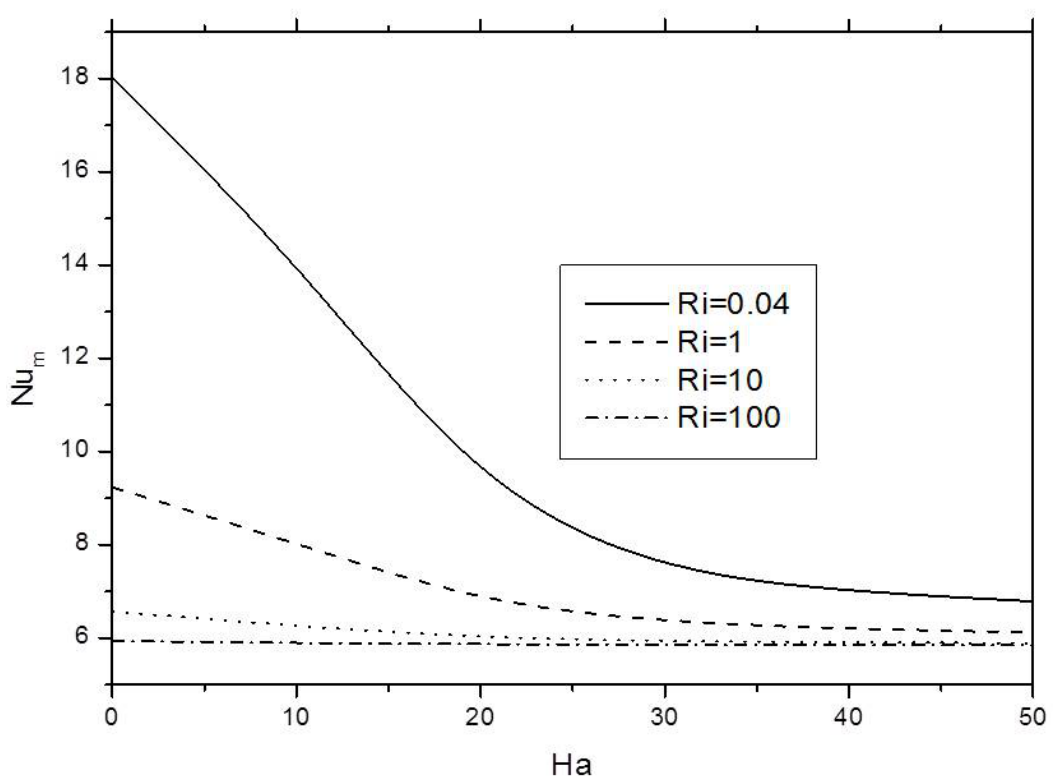

FIG. 15: Profiles of the average Nusselt number for a micropolar $\mathrm{Cu}$-water nanofluid with different values of the Hartmann $\mathrm{Ha}$ and Richardson Ri numbers at $D=0.5, K=0.5$, $\Phi=0^{0}, \phi=0.05$, and $B=0.5$

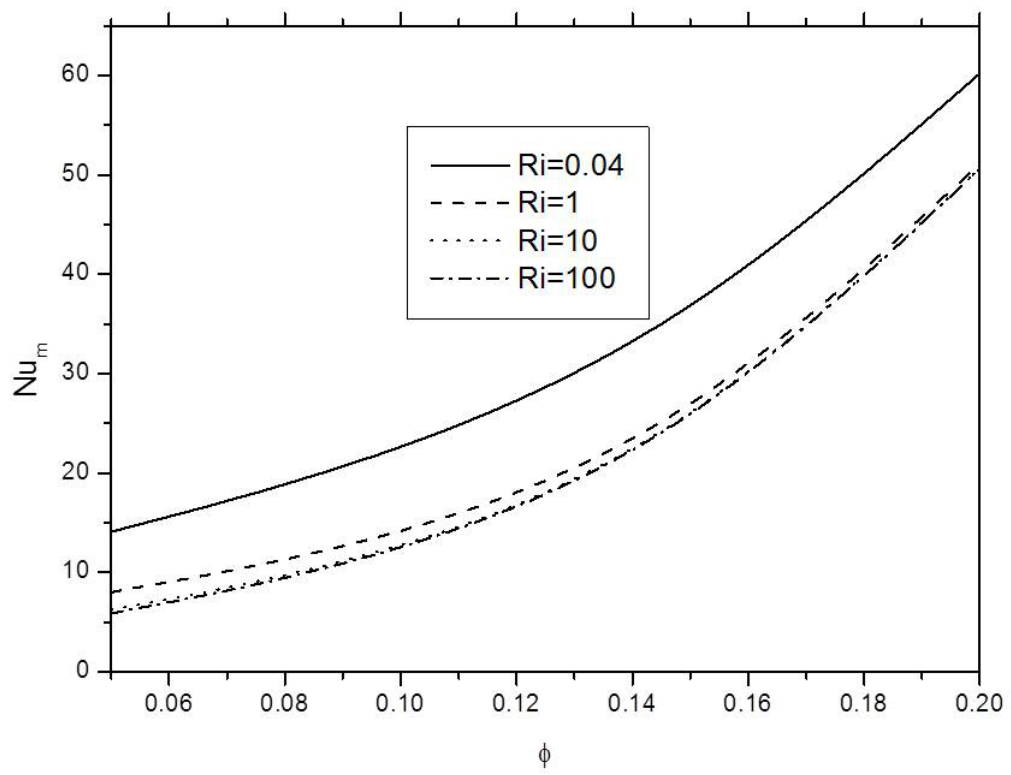

FIG. 16: Profiles of the average Nusselt number for a micropolar $\mathrm{Cu}$-water nanofluidwith different values of the solid volume fraction and the Richardson number Ri at $D=0.5$, $K=0.5, \Phi=0^{\circ}$, Ha $=10$, and $B=0.5$ 


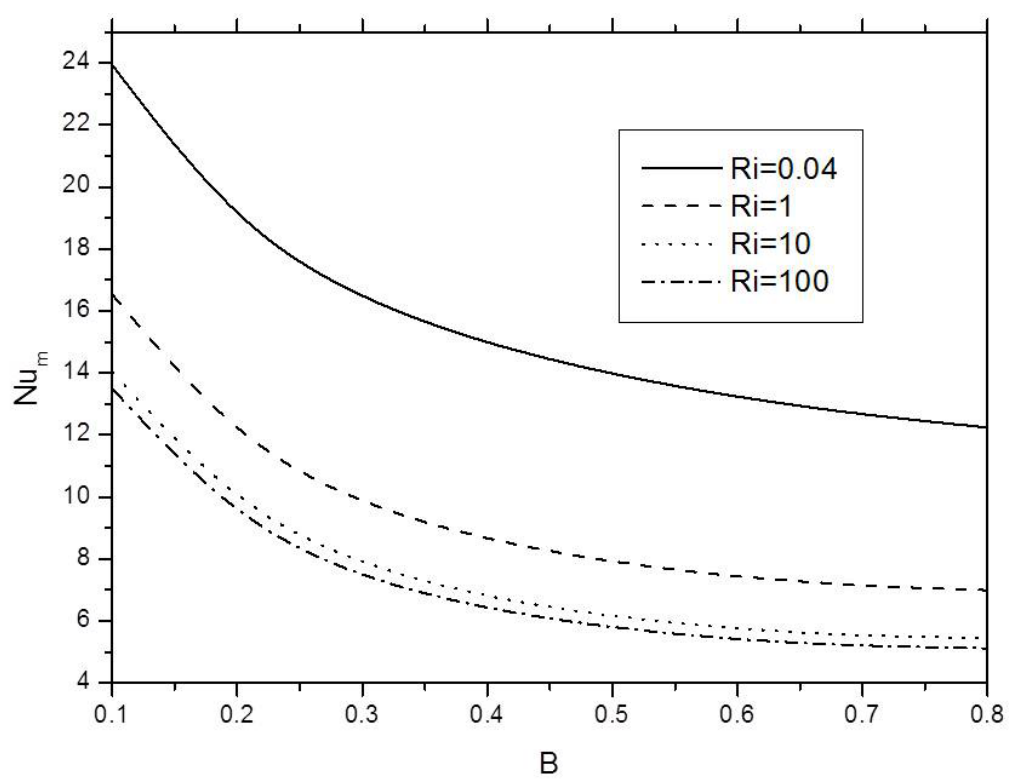

FIG. 17: Profiles of the average Nusselt number for a micropolar $\mathrm{Cu}$-water nanofluid with different values of the dimensionless length of the heat source $B$ and the Richardson number Ri at $D=0.5, K=0.5, \Phi=0^{\circ}$, Ha $=10$, and $\phi=0.05$

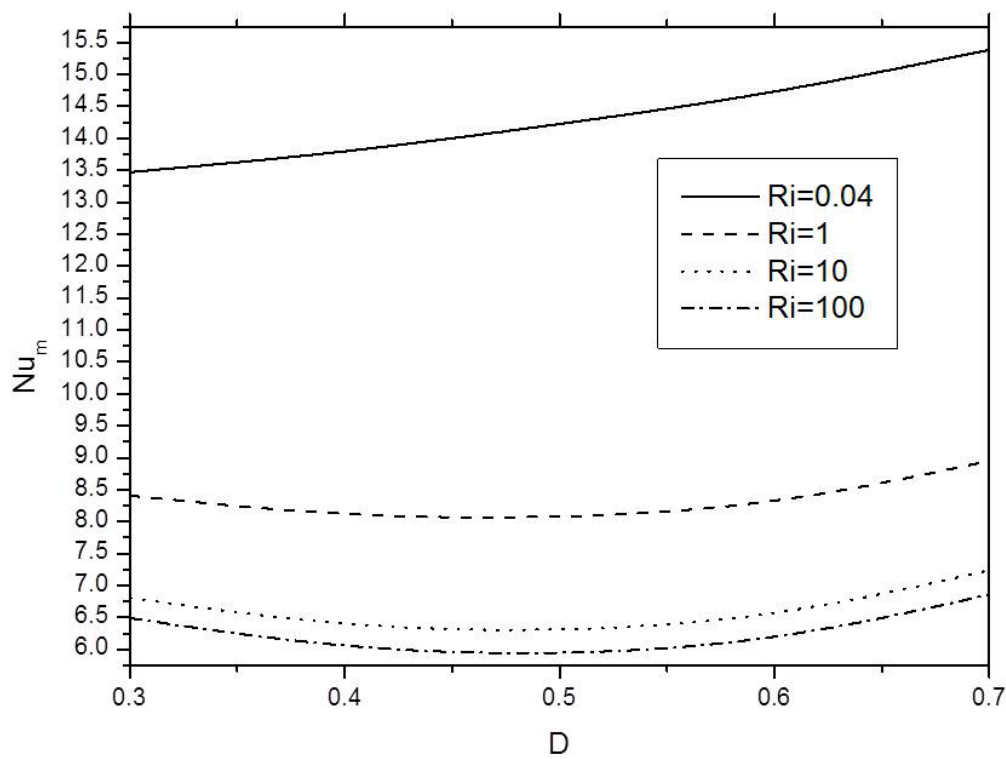

FIG. 18: Profiles of the average Nusselt number for a micropolar $\mathrm{Cu}$-water nanofluid with different values of the dimensionless location of the heat source $D$ and the Richardson number Ri at $B=0.5, K=0.5, \Phi=0^{\circ}$, Ha $=10$, and $\phi=0.05$ 


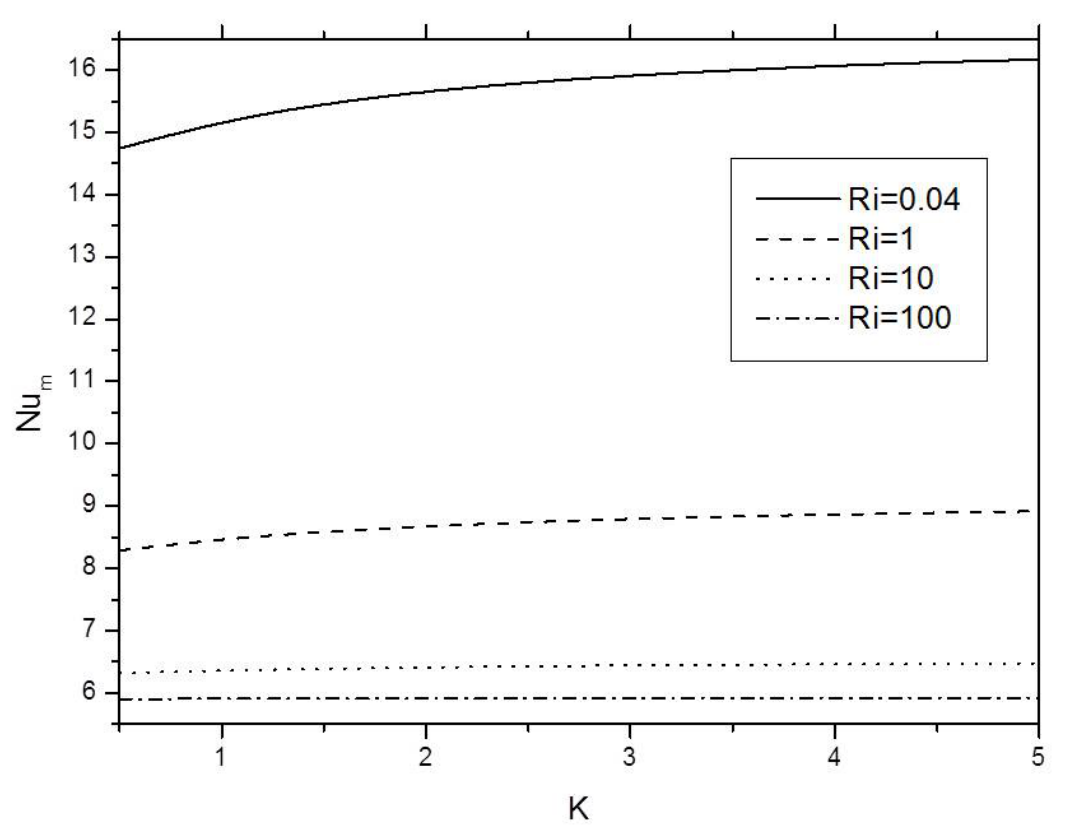

FIG. 19: Profiles of the average Nusselt number for a micropolar $\mathrm{Cu}$-water nanofluid with different values of the dimensionless viscosity $K$ and the Richardson number $\mathrm{Ri}$ at $B=0.5$, $D=0.5, \Phi=0^{\circ}$, Ha $=10$, and $\phi=0.05$

\subsection{Effect of Solid Volume Fraction}

Figure 3 explains the contours of streamlines (a), isotherms (b), and isolines of microrotation (c) of pure water $(\phi=0)$ and micropolar $\mathrm{Cu}$-water nanofluid $(\phi=0.2)$ for various solid volume fractions at $\Phi=0^{\circ}, K=0.5, D=0.5, B=0.5$, and $\mathrm{Ha}=0$ (i.e., the magnetic field effect is negligible) for natural- and forced-convection cases. The Reynolds number is taken as $\mathrm{Re}=10$ for the forced-convection case. In general, the flow field can be represented by two recirculating clockwise and counterclockwise vortices of higher strength adjacent to the cold left and right sidewalls of the trapezoidal enclosure. The flow field inside the enclosure is created adjacent to the hot region in the bottom wall of the enclosure due to the heat source effect and then moves adjacent to the left cold sidewall and then impacts with the adiabatic top wall, which leads to a change in its direction near the cold right sidewall. This repeated motion leads to the production of primary two recirculating vortices inside the trapezoidal enclosure. It can be seen from Fig. 3 that, when the solid volume fraction of nanoparticles increases from $\phi=0$ (i.e., pure fluid) to $\phi=0.2$ for the nanofluid, the circulation intensity decreases strongly for the natural-convection case and slightly for the forced-convection case. The reason for this behavior is that the high quantities of nanoparticles volume fraction (i.e., $\phi=0.2$ ) cause a significant increase in the fluid viscosity and as a result the flow velocity decreases. Moreover, for the natural-convection case, it can be ob- 
served that the flow field is distributed uniformly and covers all the regions of the trapezoidal enclosure. This is because the buoyancy force has a strong influence compared with the shear force, while for the forced-convection case, the shear force due to the moving upper lid has a stronger influence compared with the buoyancy force. Therefore, the flow field begins to move and accumulate near the moving upper lid due to this reason. With respect to isotherms, there is a clear accumulation of them adjacent to the heat source location at the bottom wall of the enclosure. This is due to the strong temperature gradient in the vertical direction which indicates that the convection heat transfer becomes more significant compared to the conduction heat transfer. It is interesting to mention that the effect of the solid volume fraction on the isotherms is slight for the natural-convection case. But for the forced-convection case, it can be seen that when the enclosure is filled with a nanofluid (i.e., $\phi=0.2$ ), the effect of the conduction becomes more dominant. Since the isotherm contours take a uniform shape, a slight disturbance is observed in this case. With respect to the isolines of microrotation, it can be seen from the results of Fig. 3 that as the solid volume fraction of nanoparticles increases from $\phi=0$ (i.e., pure fluid) to $\phi=0.2$ for the nanofluid, the strength of the microrotation increases. This behavior can be seen for both natural- and forced-convection cases. The reason for this behavior can be explained by the fact that the total amount of microrotation is increased, since the number of nanoparticles increases and consequently the total microrotation in the enclosure is increased.

\subsection{Effect of the Dimensionless Length of the Heat Source $B$}

Figure 4 shows the contours of streamlines (a), isotherms (b), and isolines of microrotation (c) of a micropolar $\mathrm{Cu}$-water nanofluid for various heat source lengths at $\mathrm{Ri}=1, \phi=0.05, \Phi=0^{\circ}, K=0.5, D=0.5$, and $\mathrm{Ha}=0$. In this case, the forced-convection effect is approximately equivalent to the natural-convection effect, i.e., $\mathrm{Ri}=1$, or, in other words, the shear force has the same influence as the buoyancy force. The results show that as the heat source length increases from $B=0.2$ to $B=0.8$, it can be seen that the flow field inside the enclosure consists of single primary counterclockwise vortices that cover the entire size of the enclosure. It can be concluded also that the flow field circulation and isolines of microrotation remain symmetrical when the heat source length increases. This behavior is due to the constant location of the heat source in the middle of the enclosure bottom wall, i.e., $D=0.5$. With respect to the isotherms, it can be noticed from the results of Fig. 4, that when the heat source length increases, the isotherms begin to distribute strongly and rapidly inside the enclosure especially at the heat source location. The reason for this behavior is due to the increase in the heat generation rate which is produced by the longer heat source. This leads to an increase in the buoyancy force effect and increases the flow circulation and the convection effects in the enclosure. Moreover, the increase in the heat generation rate

Volume 9, Issue 4, 2018 
increases the surface temperature of the heat source and as a result, the effect of the natural convection becomes stronger when the heat source length increases.

\subsection{Effect of Hartmann Number Ha}

Figure 5 illustrates the contours of streamlines (a), isotherms (b), and isolines of microrotation (c) of a micropolar $\mathrm{Cu}$-water nanofluid for various Hartmann numbers $\mathrm{Ha}$ at $\mathrm{Ri}=1, \phi=0.05, \Phi=0^{\circ}, K=0.5, D=0.5$, and $B=0.5$. In general, the flow field can be represented by single primary recirculating counterclockwise vortices adjacent to the cold left and right sidewalls of the trapezoidal enclosure. When the Hartmann number is zero, $\mathrm{Ha}=0$, or when the effect of the magnetic field is absent, the strength of circulation and microrotation is strong, since the buoyancy force due to the natural-convection effect and the shear force due to the forced-convection effect are dominant in the enclosure. It can be observed that the streamlines and isolines of microrotation are very crowded near the cold left and right sidewalls, since the mixed-convection heat transfer plays an important role in the absence of the magnetic field effect. The same phenomena can be seen when the Hartmann number is small, i.e., Ha $=5$ and 10, respectively, since the effect of the magnetic field is weak for small values of the Hartmann number. When the Hartmann number increases, i.e., Ha $=20$, the Lorentz force, which is created due to the magnetic field effect, becomes greater than the buoyancy and shear forces effect which causes to reduce the flow circulation and microrotation intensities and as a result, the convection effect begins to diminish. In fact, the existence of the magnetic field within the enclosure causes a force called a Lorentz force, which works opposite to the flow direction and it resists the flow. Therefore, it can be concluded from the results of Fig. 5 that the mixed-convection effect begins to decrease as the Hartmann number increases. With respect to isotherms, when the Hartmann number is zero, $\mathrm{Ha}=0$, or small, i.e., $\mathrm{Ha}=5$ and 10 , the isotherms are highly concentrated adjacent to the bottom wall where the heat source exists in order to develop a thermal boundary layer. In this case, the heat is transferred due to convection. But as the Hartmann number increases, i.e., Ha $=20$, the concentrated region of isotherms adjacent to the hot region in the bottom wall becomes less compressed and the isothermal lines become smooth, merely symmetrical, and clearly parallel near the cold right and left sidewalls. This is due to the increase in

the effect of the magnetic field, and in this case, the heat is transferred inside the enclosure by conduction.

\subsection{Effect of Richardson Number Ri}

Figure 6 illustrates the contours of streamlines (a), isotherms (b), and isolines of microrotation (c) of a micropolar $\mathrm{Cu}$-water nanofluid for various Richardson numbers $\mathrm{Ri}$ at $\mathrm{Ha}=10, \phi=0.05, \Phi=0^{\circ}, K=0.5, D=0.5$, and $B=0.5$. When the Richardson number is low, $\mathrm{Ri}=0.04$, the effect of the upper lid-driven wall is dominant, and the streamlines and isolines of microrotation are greatly adjacent to 
each other. In this case, the flow is derived by the forced-convection mechanism. Moreover, for low values of Richardson number, $\mathrm{Ri}=0.04$, the existence of the heat source has no clear effect on the streamlines and isolines of microrotation, when the Richardson number is unity, $\mathrm{Ri}=1.0$, or when the forced-convection effect is approximately equivalent to the natural-convection effect. In this case, the shear force has the same influence as the buoyancy force. It can be seen from the results that the behavior of the flow field is approximately similar to that presented when the Richardson number is low, $\mathrm{Ri}=0.04$. The only difference which can be seen is that the vortices core begins to increase in size and moves towards the center of the enclosure, if they are compared with the vortices observed when the Richardson number is very low, $\mathrm{Ri}=0.04$. The same phenomena can be seen with respect to the isolines of microrotation, but now, when the Richardson number is high, $\mathrm{Ri}=10$ and 100, or when the forced-convection effect is less than the natural-convection effect. In this case, the buoyancy force is greater than the shear force and becomes more dominant. Therefore, the vortices core begins to increase further, and the existence of the heat source has a noticeable effect on the streamlines and isolines of microrotation. Furthermore, the results of Fig. 6 indicate that the maximum values of the stream function occur when the Richardson number is low, $\mathrm{Ri}=0.04$. With respect to the isotherms, when the Richardson number is low, $\mathrm{Ri}=0.04$, there is a clear accumulation of isotherms adjacent to the heat source location at the bottom wall of the enclosure. This is due to the strong temperature gradient in the vertical direction, which indicates that the convection heat transfer becomes more significant compared to the conduction heat transfer. In general, when the Richardson number is low, i.e., Reynolds number is high, the isotherm contours take a nonuniform shape and a high disturbance can be seen in it where the convection heat transfer is dominant. When the Richardson number is unity, $\mathrm{Ri}=1.0$, the disturbance in the isotherm contours begins to increase gradually if they are compared with the corresponding isotherm contours for the low Richardson number $\mathrm{Ri}=0.04$. The thermal energy inside the enclosure is transferred also by convection. This can be observed from the nonuniform isotherm lines adjacent to the cold left and right sidewalls of the enclosure. Now, when the Richardson number increases to $\mathrm{Ri}=10$ and 100 , the isotherms begin to distribute uniformly parallel to the cold left and right sidewalls indicating that the conduction is the dominant mechanism for heat transfer in the enclosure.

\subsection{Effect of Nanofluid Type}

In this part of the study, the effect of different types of nanofluids ( $\mathrm{Ag}, \mathrm{Al}_{2} \mathrm{O}_{3}$, and $\mathrm{TiO}_{2}$ ) and the pure water is considered. Figure 7 shows the contours of streamlines (a), isotherms (b), and isolines of microrotation (c) for different nanofluids and pure water at $\mathrm{Ha}=10, \mathrm{Ri}=1, \phi=0.05, \Phi=0^{\circ}, K=0.5, D=0.5$, and $B=0.5$. It is found that the largest value of the flow circulation can be obtained by adding Ag compared to other nanoparticles. On the contrary, $\mathrm{TiO}_{2}$ gives

Volume 9, Issue 4, 2018 
the lowest value of the flow circulation. This is due to the fact that the thermal energy transport from the heated region is higher for nanoparticles with higher thermal conductivities. On the other hand, it can be seen also that the values of the flow circulation for all types of nanoparticles are higher than that of pure water. This is a logical result, since the thermal conductivity of the pure water is smaller than the thermal conductivity of the considered nanoparticles, as shown in Table 1 . With respect to the isotherms, it can be seen from Fig. 7 that there is a clear accumulation of them adjacent to the heat source location at the bottom wall of the enclosure when the Ag-water nanofluid is used. This is due to the high energy transport in the vertical direction which indicates that the convection heat transfer becomes more significant compared to the conduction heat transfer when the Ag-water nanofluid is used. But when the pure water is used, the isotherm contours take a uniform shape especially adjacent to the heat source location, and a slight disturbance can be seen in it indicating that the conduction heat transfer is dominant.

\subsection{Velocity Profiles}

In order to have a better understanding of the flow behavior in the present work, the vertical velocity profiles along the midsection of the enclosure $(Y=0.5)$ are presented in Fig. 8 for a micropolar $\mathrm{Cu}$-water nanofluid at $B=0.5, \mathrm{Ri}=1$, $D=0.5, \Phi=0^{\circ}, K=0.5$, and $\phi=0.05$. It is shown that the flow begins from zero, then increases and after that begins to decrease. Therefore, a positive velocity profiles can be observed in the upper region of the trapezoidal enclosure and then a positive velocity profiles can be observed in the middle of the trapezoidal enclosure. After that, negative velocity profiles can be seen. This behavior is due to the high circulation of the fluid flow which can be observed when the Hartmann number is zero or low. Therefore, when $X \leq 0.5$, the maximum vertical velocity profiles occur when the Hartmann number is zero. A similar but a reverse velocity profiles can be observed when $X>0.5$. Furthermore, it is found that as the Hartmann number increases to $\mathrm{Ha}=50$, the velocity profiles approach the enclosure vertical centerline. This is due to the significant drop in the flow field circulation when the Hartmann number is high, i.e., Ha $=50$. Figure 9 shows the profiles of the vertical velocity component at the enclosure midsection for a micropolar $\mathrm{Cu}$-water nanofluid at $B=0.5$, $\mathrm{Ha}=10, D=0.5, \Phi=0^{\circ}, K=0.5$, and $\phi=0.05$. Different velocity profiles are observed which indicate the direction of the flow rotation. The results indicate that when the Richardson number is low, i.e., $\mathrm{Ri}=0.04$, the velocity profiles vary significantly across the midsection of the enclosure which indicates a high circulation zone in the enclosure. But it can be seen that the velocity profiles begin to match each other as the Richardson number increases. This is due to the reduction in the velocity of the upper lid-driven wall.

\subsection{Local Nusselt Number Results}

Figure 10 illustrates the profiles of the local Nusselt number along the heat source for a micropolar $\mathrm{Cu}$-water nanofluid with different values of dimensionless length 
of the heat source $B$ at $D=0.5, K=0.5, \mathrm{Ri}=1, \phi=0.05, \Phi=0^{\circ}$, and $\mathrm{Ha}=0$. The results indicate that the local Nusselt number along the heat source decreases as the heat source length increases from $B=0.2$ to $B=0.8$. The reason of this behavior is due to the increase in the maximum temperature of the heat source by increasing its length which leads to a decrease of the local Nusselt number. Figure 11 shows profiles of the local Nusselt number along the heat source for a micropolar $\mathrm{Cu}$-water nanofluid with different values of the Hartmann number $\mathrm{Ha}$ at $D=0.5$, $K=0.5, \mathrm{Ri}=1, \phi=0.05, \Phi=0^{\circ}$, and $B=0.5$. It can be seen from this figure that the local Nusselt number along the heat source decreases when the Hartmann number increases. This is due to the effect of magnetic field which becomes very significant with increasing Hartmann number, decreases the strength of flow circulation and reduces the temperature gradient near the hot region in the bottom wall, and for this reason the local Nusselt number begins to decrease. Figure 12 displays

the profiles of the local Nusselt number along the heat source for a micropolar $\mathrm{Cu}$-water nanofluid with different values of the Richardson number $\mathrm{Ri}$ at $D=0.5$, $K=0.5$, Ha $=10, \phi=0.05, \Phi=0^{\circ}$, and $B=0.5$. It can be observed that the local Nusselt number has a large value when the Richardson number is low, i.e., in the forced-convection-dominant region. The reason of this behavior is due to the high effect of the Reynolds number when the Richardson number is low. Therefore the velocity of the upper lid-driven wall increases significantly and leads to an increase in the forced-convection effect on the local Nusselt number. On the other hand, when the Richardson number is high, $\mathrm{Ri}=100$, i.e., in the natural-convection-dominant region, the velocity of the upper lid-driven wall begins to decrease significantly and leads to a decrease in the forced convection and the upper lid-driven wall effects on the local Nusselt number. Figure 13 displays the profiles of the local Nusselt number along the heat source for different nanofluids and pure water at $D=0.5, K=0.5, \mathrm{Ha}=10, \mathrm{Ri}=1, \phi=0.05, \Phi=0^{\circ}$, and $B=0.5$. It is observed that the largest value of the local Nusselt number along the heat source can be obtained by adding copper $(\mathrm{Cu})$, while the smallest value of it can be obtained by adding silver (Ag) nanoparticles. Moreover, symmetrical profiles of the local Nusselt number along the heat source are obtained for all nanofluids and pure water.

\subsection{Average Nusselt Number Results}

Figure 14 illustrates the profiles of the average Nusselt number for a micropolar $\mathrm{Cu}$-water nanofluid with different values of the magnetic field orientation angle and the Richardson number $\mathrm{Ri}$ at $D=0.5, K=0.5$, $\mathrm{Ha}=10, \phi=0.05$, and $B=0.5$. As illustrated in Fig. 14, the average Nusselt number increases as the Richardson number decreases. The reason of this behavior is due to the increase in the Reynolds number and the forced-convection effect for low values of the Richardson number, i.e., $\mathrm{Ri}=0.04$, and this leads to an increase in the average Nusselt number. With respect to the effect of the magnetic field orientation angle, it is found that when the Richardson number is high, $\mathrm{Ri}=10$ and 100 , the change

Volume 9, Issue 4, 2018 
in the magnetic field orientation angles does not have a significant influence on the heat transfer rate represented by the average Nusselt number. But when the Richardson number is low, $\mathrm{Ri}=0.04$ and 1.0, the results of Fig. 14 indicate that the average Nusselt number varies significantly with the variation of the magnetic field orientation angles. Figure 15 displays the profiles of the average Nusselt number for a micropolar $\mathrm{Cu}$-water nanofluid with different values of the Hartmann and Richardson numbers at $D=0.5, K=0.5, \Phi=0^{\circ}, \phi=0.05$, and $B=0.5$. It is found that as the Hartmann number increases, the average Nusselt number decreases. This is due to the increase of the effect of the magnetic force when the Hartmann number increases. Thus, the increased magnetic field reduces the rate of heat transfer due to the reduction of the flow circulation and the increase in the thermal boundary layer thickness. Also, the maximum value of the average Nusselt numbers occurs when the Hartmann number is zero, i.e., the magnetic field is absent. Therefore, the high average Nusselt numbers correspond to the low Hartmann number and vice versa. This occurs because the effect of the magnetic field becomes negligible when the Hartmann number is zero. Therefore the flow circulation strength and the temperature gradient increase, and for this reason the average Nusselt number increases. An opposite behavior can be found when the Hartmann number increases. This phenomena can be seen clearly when the Richardson number is low, $\mathrm{Ri}=0.04$ and 1.0 , but when the Richardson number is high, $\mathrm{Ri}=10$ and 100 , it is found that there is a linear variation between the average Nusselt number and the Hartmann number. This is a logical result due to the weak effect of the forced-convection effect when the Richardson number is high. Figure 16 explains the profiles of the average Nusselt number for a micropolar $\mathrm{Cu}$-water nanofluid with different values of the solid volume fraction and the Richardson number at $D=0.5, K=0.5, \Phi=0^{\circ}$, $\mathrm{Ha}=10$, and $B=0.5$. As can be seen from Fig. 16, the average Nusselt number increases when the Richardson number decreases for the same reasons explained previously. This behavior can be seen for all values of the solid volume fraction. At $\mathrm{Ri}=0.04$, the average Nusselt number increases dramatically. Moreover, as illustrated in Fig. 16, the average Nusselt number increases when the solid volume fraction increases. The reason of this behavior is due to the increase of the thermal conductivity of the nanofluid when the solid volume fraction increases. This leads to the increase in the rate of the heat transfer and, as a result, the average Nusselt number increases. Figure 17 shows the profiles of the average Nusselt number for a micropolar $\mathrm{Cu}$-water nanofluid with different values of the dimensionless length of the heat source $B$ and the Richardson number at $D=0.5, K=0.5, \Phi=0^{\circ}$, Ha $=10$, and $\phi=0.05$. The results indicate that the average Nusselt number decreases as the heat source length increases from $B=0.1$ to $B=0.8$ for various values of the Richardson number. The reason of this behavior is due to the increase in the maximum temperature of the heat source by increasing its length, which leads to a decrease in the average Nusselt number. Therefore it can be seen from Fig. 17, that the maximum average Nusselt number occurs for shorter length of the heat source, i.e., $B=0.1$. Figure 18 
displays the profiles of the average Nusselt number for a micropolar $\mathrm{Cu}$-water nanofluid with different values of the dimensionless location of the heat source $D$ and the Richardson number at $B=0.5, K=0.5, \Phi=0^{\circ}$, Ha $=10$, and $\phi=0.05$. It can be seen that when the Richardson number is low, $\mathrm{Ri}=0.04$, the average Nusselt number increases as the dimensionless location of the heat source increases. On the other hand, it can be seen from Fig. 18 that there is a slight decrease in the average Nusselt number for the dimensionless location of the heat source $D$ in the range $0.4 \leq D \leq 0.6$. This behavior can be seen when the Richardson number in the range $1.0 \leq \mathrm{Ri} \leq 100$. Figure 19 illustrates the profiles of the average Nusselt number for a micropolar $\mathrm{Cu}$-water nanofluid with different values of the dimensionless viscosity $K$ and the Richardson number at $B=0.5, D=0.5$, $\Phi=0^{\circ}$, Ha $=10$, and $\phi=0.05$. It can be seen that when the Richardson number is low, $\mathrm{Ri}=0.04$ and 1.0, the average Nusselt number increases as the dimensionless viscosity increases. But, when the Richardson number is high, $\mathrm{Ri}=10$ and 100 , it is found that there is a slight variation between the average Nusselt number and the dimensionless viscosity.

\section{CONCLUSIONS}

The following conclusions can be drawn from the present work results.

1. When the solid volume fraction of nanoparticles increases, the circulation intensity decreases strongly for the natural-convection case and slightly for the forced-convection case. Moreover, the effect of the solid volume fraction on the isotherms is higher in the forced-convection case than in the natural-convection case.

2. When the heat source length increases, both the circulation and microrotation of the flow field remain symmetrical, while the isotherms begin to distribute strongly and rapidly inside the enclosure especially at the heat source location.

3. When the Hartmann number is high, the intensity of the applied magnetic field increases. This causes the reduction of the circulation and microrotation of the flow field, and the heat is transferred inside the enclosure by conduction.

4. Maximum vertical velocity profiles occur when and the Hartmann number is zero. Also, the results indicate that when the Richardson number is low, i.e., $\mathrm{Ri}=0.04$, the velocity profiles vary significantly across the midsection of the enclosure which indicates the presence of a high circulation zone in the enclosure.

5. Maximum values of the stream function occur when the Richardson number is low, $\mathrm{Ri}=0.04$, and the convection heat transfer is dominant in this case.

6. The local Nusselt number along the heat source decreases when the Hartmann number, Richardson number, and the heat source length increase.

7. The largest value of the local Nusselt number along the heat source can be obtained by adding copper $(\mathrm{Cu})$, while the smallest value of it can be obtained by adding silver (Ag) nanoparticles.

Volume 9, Issue 4, 2018 
8. The average Nusselt number increases when the Richardson number decreases and the solid volume fraction increases, while it decreases as the heat source length increases.

9. When the Richardson number is low, $\mathrm{Ri}=0.04$, the average Nusselt number increases as the dimensionless location of the heat source increases. But there is a slight decrease in the average Nusselt number at the dimensionless location of the heat source in the range $0.4 \leq D \leq 0.6$. This behavior can be seen when the Richardson number is in the range $1.0 \leq \mathrm{Ri} \leq 100$.

10. When the Hartmann number increases, the average Nusselt number decreases. This occurs when the Richardson number is low, $\mathrm{Ri}=0.04$ and 1.0 , but when the Richardson number is high, $\mathrm{Ri}=10$ and 100, a linear variation between the average Nusselt number and the Hartmann number is found.

11. When the Richardson number is low, $\mathrm{Ri}=0.04$ and 1.0, the average Nusselt number increases as the dimensionless viscosity increases. But when the Richardson number is high, $\mathrm{Ri}=10$ and 100, a slight variation between the average Nusselt number and the dimensionless viscosity can be seen.

12. The flow circulation and the convection heat transfer are increased by adding Ag nanoparticles to the base fluid (i.e., water), while pure water gives small values of the flow circulation and increases the effect of the conduction heat transfer.

13. The strength of the microrotation increases when the solid volume fraction increases.

\section{REFERENCES}

Ahmadi, G., Self-Similar Solution of Imcompressible Micropolar Boundary Layer Flow over a SemiInfinite Plate, Int. J. Eng. Sci., vol. 14, no. 7, pp. 639-646, 1976.

Alinia, M., Ganji, D.D., and Gorji-Bandpy, M., Numerical Study of Mixed Convection in an Inclined Two Sided Lid Driven Cavity Filled with Nanofluid Using Two-Phase Mixture Model, Int. Commun. Heat Mass Transf., vol. 38, no. 10, pp. 1428-1435, 2011.

Aminossadati, S.M. and Ghasemi, B., Natural Convection Cooling of a Localised Heat Source at the Bottom of a Nanofluid-Filled Enclosure, Eur. J. Mech. - B/Fluids, vol. 28, no. 5, pp. 630-640, 2009.

Arani, A.A., Sebdani, S.M., Mahmoodi, M., Ardeshiri, A., and Aliakbari, M., Numerical Study of Mixed Convection Flow in a Lid-Driven Cavity with Sinusoidal Heating on Sidewalls Using Nanofluid, Superlattices Microstructures, vol. 51, no. 6, pp. 893-911, 2012.

Basak, T., Roy, S., Singh, A., and Pop, I., Finite Element Simulation of Natural Convection Flow in a Trapezoidal Enclosure Filled with Porous Medium Due to Uniform and Non-Uniform Heating, Int. J. Heat Mass Transf., vol. 52, nos. 1-2, pp. 70-78, 2009.

Bhattacharya, M., Basak, T., Oztop, H.F., and Varol, Y., Mixed Convection and Role of Multiple Solutions in Lid-Driven Trapezoidal Enclosures, Int. J. Heat Mass Transf., vol. 63, pp. 366-388, 2013.

Chamkha, A.J. and Abu-Nada, E., Mixed Convection Flow in Single- and Double-Lid Driven Square Cavities Filled with Water- $\mathrm{Al}_{2} \mathrm{O}_{3}$ Nanofluid: Effect of Viscosity Models, Eur. J. Mech. - B/Fluids, vol. 36, pp. 82-96, 2012. 
Choi, S., Enhancing Thermal Conductivity of Fluids with Nanoparticles, in D.A. Siginer and H.P. Wang, Developments and Applications of Non-Newtonian Flows, FED-vol. 231/MD-vol. 65, pp. 99-105, New York: ASME, 1995.

Eringen, A.C., Theory of Micropolar Fluids, DTIC Document, 1965.

Esfe, M.H., Ghadi, A.Z., and Noroozi, M.J., Numerical Simulation of Mixed Convection within Nanofluid-Filled Cavities with Two Adjacent Moving Walls, Trans. Can. Soc. Mech. Eng., vol. 37, no. 4, pp. 1073-1089, 2013.

Ferdows, M., Khan, M., Alam, M., and Sun, S., MHD Mixed Convective Boundary Layer Flow of a Nanofluid through a Porous Medium Due to an Exponentially Stretching Sheet, Math. Probl. Eng., vol. 2012, 2012.

Garoosi, F., Garoosi, S., and Hooman, K., Numerical Simulation of Natural Convection and Mixed Convection of the Nanofluid in a Square Cavity Using Buongiorno Model, Powder Technol., vol. 268, pp. 279-292, 2014.

Godson, L., Raja, B., Lal, D.M., and Wongwises, S., Experimental Investigation on the Thermal Conductivity and Viscosity of Silver-Deionized Water Nanofluid, Exp. Heat Transf., vol. 23, no. 4, pp. 317-332, 2010.

Hasib, M.H., Hossen, M.S., and Saha, S., Effect of Tilt Angle on Pure Mixed Convection Flow in Trapezoidal Cavities Filled with Water- $\mathrm{Al}_{2} \mathrm{O}_{3}$ Nanofluid, Proc. Eng., vol. 105, pp. 388-397, 2015.

Hsu, T.-H. and Wang, S.-G., Mixed Convection of Micropolar Fluids in a Cavity, Int. J. Heat Mass Transf., vol. 43, no. 9, pp. 1563-1572, 2000.

Hussain, S.H. and Hussein, A.K., Mixed Convection Heat Transfer in a Differentially Heated Square Enclosure with a Conductive Rotating Circular Cylinder at Different Vertical Locations, Int. Commun. Heat Mass Transf., vol. 38, no. 2, pp. 263-274, 2011.

Hussein, A.K., Ahmed, S.E., Mohammed, H., and Khan, W.A., Mixed Convection of Water-based Nanofluids in a Rectangular Inclined Lid-Driven Cavity Partially Heated from Its Left Sidewall, $J$. Comput. Theor. Nanosci., vol. 10, no. 9, pp. 2222-2233, 2013.

Hussein, A.K., Applications of Nanotechnology in Renewable Energies - A Comprehensive Overview and Understanding, Renew. Sustain. Energy Rev., vol. 42, pp. 460-476, 2015.

Kalteh, M., Javaherdeh, K., and Azarbarzin, T., Numerical Solution of Nanofluid Mixed Convection Heat Transfer in a Lid-Driven Square Cavity with a Triangular Heat Source, Powder Technol., vol. 253, pp. 780-788, 2014.

Keshtkar, M.M., Esmaili, N., and Ghazanfari, M.R., Effect of Heat Source/Sink on MHD Mixed Convection Boundary Layer Flow on a Vertical Surface in a Porous Medium Saturated by a Nanofluid with Suction or Injection, Int. J. Eng. Sci., vol. 4, pp. 1-11, 2014.

Khanafer, K., Vafai, K., and Lightstone, M., Buoyancy-Driven Heat Transfer Enhancement in a Two-Dimensional Enclosure Utilizing Nanofluids, Int. J. Heat Mass Transf., vol. 46, no. 19, pp. 3639-3653, 2003.

Kolsi, L., Hussein, A.K., Borjini, M.N., Mohammed, H., and Aissia, H.B., Computational Analysis of Three-Dimensional Unsteady Natural Convection and Entropy Generation in a Cubical Enclosure Filled with Water- $\mathrm{Al}_{2} \mathrm{O}_{3}$ Nanofluid, Arab. J. Sci. Eng., vol. 39, no. 11, pp. 7483-7493, 2014.

Mahmoud, M. and Waheed, S., Effects of Slip and Heat Generation/Absorption on MHD Mixed Convection Flow of a Micropolar Fluid over a Heated Stretching Surface, Math. Probl. Eng., vol. 2010, 2010.

Mamun, M., Tanim, T., Rahman, M., Saidur, R., and Nagata, S., Mixed Convection Analysis in Trapezoidal Cavity with a Moving Lid, Int. J. Mech. Mater. Eng., vol. 5, no. 1, pp. 18-28, 2010.

Volume 9, Issue 4, 2018 
Mansour, M. and Ahmed, S.E., Mixed Convection in Double Lid-Driven Enclosures Filled with $\mathrm{Al}_{2} \mathrm{O}_{3}$-Water Nanofluid, J. Thermophys. Heat Transf., vol. 27, no. 4, pp. 707-718, 2013.

Mansour, M.A., Mohamed, R.A., Abd-Elaziz, M.M., and Ahmed, S.E., Numerical Simulation of Mixed Convection Flows in a Square Lid-Driven Cavity Partially Heated from Below Using Nanofluid, Int. Commun. Heat Mass Transf., vol. 37, no. 10, pp. 1504-1512, 2010.

Muthtamilselvan, M. and Doh, D.H., Mixed Convection of Heat Generating Nanofluid in a Lid-Driven Cavity with Uniform and Non-Uniform Heating of Bottom Wall, Appl. Math. Model., vol. 38, no. 13, pp. 3164-3174, 2014.

Oztop, H.F. and Abu-Nada, E., Numerical Study of Natural Convection in Partially Heated Rectangular Enclosures Filled with Nanofluids, Int. J. Heat Fluid Flow, vol. 29, no. 5, pp. 1326-1336, 2008.

Pak, B.C. and Cho, Y.I., Hydrodynamic and Heat Transfer Study of Dispersed Fluids with Submicron Metallic Oxide Particles, Exp. Heat Transf., vol. 11, no. 2, pp. 151-170, 1998.

Rees, D.A.S. and Pop, I., Free Convection Boundary-Layer Flow of a Micropolar Fluid from a Vertical Flat Plate, IMA J. Appl. Math., vol. 61, pp. 179-197, 1998.

Sebdani, S.M., Mahmoodi, M., and Hashemi, S.M., Effect of Nanofluid Variable Properties on Mixed Convection in a Square Cavity, Int. J. Therm. Sci., vol. 52, pp. 112-126, 2012.

Sheikhzadeh, G., Hajialigol, N., Ebrahim, Q.M., and Fattahi, A., Laminar Mixed Convection of CuWater Nanofluid in Two-Sided Lid-Driven Enclosures, J. Nanostruct., pp. 44-53, 2012.

Sivasankaran, S., Sivakumar, V., Hussein, A.K., and Prakash, P., Mixed Convection in a Lid-Driven Two-Dimensional Square Cavity with Corner Heating and Internal Heat Generation, Numer. Heat Transf., Part A: Appl., vol. 65, no. 3, pp. 269-286, 2014.

Talebi, F., Mahmoudi, A.H., and Shahi, M., Numerical Study of Mixed Convection Flows in a Square Lid-Driven Cavity Utilizing Nanofluid, Int. Commun. Heat Mass Transf., vol. 37, no. 1, pp. 79-90, 2010.

Tmartnhad, I., El Alami, M., Najam, M., and Oubarra, A., Numerical Investigation on Mixed Convection Flow in a Trapezoidal Cavity Heated from Below, Energy Convers. Manage., vol. 49, no. 11, pp. 3205-3210, 2008. 


\section{NANOSCIENCE AND TECHNOLOGY AN INTERNATIONAL JOURNAL \\ CONTENTS, VOLUME 9, 2018}

Page Range of Issues - Issue 1: 1-89; Issue 2: 91-181; Issue 3: 183-282; Issue 4: 283-372

$\underline{\text { Issue } 1}$

On the Dynamic Equilibrium of Nanobubbles in Water

S.I. Koshoridze \& Yu.K. Levin

Nanomodified Epoxy Composite Materials for Metal-Polymer Tribosystems

of Transport Vehicles

D. Zinchenko \& S. Smetankin

A Numerical Solution for Predicting the Stiffness of Nanophase Biaxially Braided

Composites

R. Parimala \& D.B. Jabaraj

Reinforcement Effects in SBR Rubber/Modified Shungite Nanocomposites

Y.V. Kornev, N.A. Semenov, A.V. Babaytsev, \& H.H. Valiev

Effects of Chemical Reaction and Slip in the Boundary Layer of MHD Nanofluid Flow through a Semi-Infinite Stretching Sheet with Thermophoresis and Brownian Motion: The Lie Group Analysis

S.K. Rawat, A.K. Pandey, \& M. Kumar

Effect of Nanosized Additives on the Improvement of the Efficiency of Composite

Fuel

V.A. Levin, I.S. Manuylovich, V.V. Markov, I.P. Bolshiyanov,

N.N. Zakharov, \& Yu.K. Levin

Model of Chemical Conversion Initiation in the Adhesive Bonding Layer during Roll Motion in the Process of Laminated Object Creation

A.G. Knyazeva

\section{Issue 2}

Nanotechnological Aspects of Temperature-Dependent Decomposition of Polymer Solutions

N.A. Bulychev, E.L. Kuznetsova, V.V. Bodryshev, \& L.N. Rabinskiy

Ohmic-Viscous Dissipation and Slip Effects on Nanofluid Flow over a Stretching Cylinder with Suction/Injection

A. Mishra, A.K. Pandey, \& M. Kumar

Modeling the Effective Dynamic Properties of Fiber Composites Modified across Length Scales

D.B. Volkov-Bogorodskiy, S.A. Lurie, \& G.I. Kriven

Systematic Study of Structural and Conductive Properties of Copper Nanotubes Modified by Ionizing Radiation

I. Kenzhina, A. Kozlovskiy, M. Kaikanov, M. Zdorovets, \& A. Tikhonov

Effect of the Phase State of a Polymer Matrix on the Degree of Reinforcement of Polymer Organoclay Nanocomposites 
Nanostructural Model of Shape Memory Alloy with Resistance Asymmetry

Behavior

165

I.V. Mishustin

\section{Issue 3}

Fluid Flow Simulation in Microporous Media on Nonuniform Grids Using the Taylor Series Expansion and Least Squares-Based Lattice Boltzmann Method

A.R. Rahmati

MHD Flow and Nonlinear Radiative Heat Transfer of a Casson Nanofluid past a Nonlinearly Stretching Sheet in the Presence of Chemical

Reaction

B.J. Gireesha, M.R. Krishnamurthy, B.C. Prasannakumara, \& R.S.R. Gorla

Effects of Stress Relieving Annealing Parameters on the Quasi-Static Compressive Properties of Closed-Cell Aluminum Calcium Foam

D. Puspitasari, T.L. Ginta, P. Puspitasari, \& M. Mustapha

Numerical Simulation of Time-Dependent Non-Newtonian Nanopharmacodynamic Transport Phenomena in a Tapered Overlapping Stenosed Artery

N. Ali, A. Zaman, M. Sajid, O. Anwar Bég, M.D. Shamshuddin,

\& A. Kadir

\section{Issue 4}

Modeling of Subgrain's Crystallographic Misorientation Distribution N.S. Kondratev \& P.V. Trusov

A Variant of Describing Adhesion Interaction in the Probe-Sample System of an Atomic-Force Microscope

V.Yu. Presnetsova, S.N. Romashin, L.Yu. Frolenkova, V.S. Shorkin, \& S.I. Yakushina

Effects of Viscous Dissipation and Heat Generation/Absorption on Nanofluid Flow over an Unsteady Stretching Surface with Thermal Radiation and Thermophoresis A.K. Pandey \& M. Kumar

MHD Mixed Convection in Trapezoidal Enclosures Filled with Micropolar Nanofluids

S.E. Ahmed, A.K. Hussein, M.A. Mansour, Z.A. Raizah, \& X. Zhang 


\section{NANOSCIENCE AND TECHNOLOGY AN INTERNATIONAL JOURNAL}

AUTHOR INDEX, VOLUME 9, 2018

\begin{tabular}{|c|c|c|}
\hline \multicolumn{3}{|c|}{ Page Range of Issues - Issue 1: 1-89; Issue 2: 91-181; Issue 3: 183-282; Issue 4: 283-372 } \\
\hline Ahmed, S.E., 343 & Kozlov, G.V., 155 & Rabinskiy, L.N., 91 \\
\hline Ali, N., 247 & Kozlovskiy, A., 139 & Rahmati, A.R., 183 \\
\hline Anwar Bég, O., 247 & Krishnamurthy, M.R., 207 & Raizah, Z.A., 343 \\
\hline Babaytsev, A.V., 31 & Kriven, G.I., 117 & Rawat, S.K., 47 \\
\hline Bodryshev, V.V., 91 & Kumar, M., 47, 99, 325 & Romashin, S.N., 299 \\
\hline Bolshiyanov, I.P., 69 & Kuznetsova, E.L., 91 & Sajid, M., 247 \\
\hline Bulychev, N.A., 91 & Levin, V.A., 69 & Semenov, N.A., 31 \\
\hline Dolbin, I.V., 155 & Levin, Yu.K., 1, 69 & Shamshuddin, M.D., 247 \\
\hline Frolenkova, L.Yu., 299 & Lurie, S.A., 117 & Shorkin, V.S., 299 \\
\hline Ginta, T.L., 231 & Mansour, M.A., 343 & Smetankin, S., 9 \\
\hline Gireesha, B.J., 207 & Manuylovich, I.S., 69 & Tikhonov, A., 139 \\
\hline Gorla, R.S.R., 207 & Markov, V.V., 69 & Trusov, P.V., 283 \\
\hline Hussein, A.K., 343 & Mishra, A., 99 & Valiev, H.H., 31 \\
\hline Jabaraj, D.B., 19 & Mishustin, I.V., 165 & Vlasov, A.N., 155 \\
\hline Kadir, A., 247 & Mustapha, M., 231 & Volkov-Bogorodskiy, D.B., \\
\hline Kaikanov, M., 139 & Pandey, A.K., 47, 99, 325 & 117 \\
\hline Karnet, Yu.N., 155 & Parimala, R., 19 & Yakushina, S.I., 299 \\
\hline Kenzhina, I., 139 & Prasannakumara, B.C., & Zakharov, N.N., 69 \\
\hline Knyazeva, A.G., 77 & 207 & Zaman, A., 247 \\
\hline Kondratev, N.S., 283 & Presnetsova, V.Yu., 299 & Zdorovets, M., 139 \\
\hline Kornev, Y.V., 31 & Puspitasari, D., 231 & Zhang, X., 343 \\
\hline Koshoridze, S.I., 1 & Puspitasari, P., 231 & Zinchenko, D., 9 \\
\hline
\end{tabular}




\section{NANOSCIENCE AND TECHNOLOGY AN INTERNATIONAL JOURNAL}

SUBJECT INDEX, VOLUME 9, 2018

\begin{tabular}{|c|c|c|}
\hline \multicolumn{3}{|c|}{ Page Range of Issues - Issue 1: 1-89; Issue 2: 91-181; Issue 3: 183-282; Issue 4: 283-372 } \\
\hline adhesion, 299 & heat treatment, 231 & phase transformations, \\
\hline adhesive bonding layer, 77 & heterogeneous hardening, & 165 \\
\hline aerodynamic tunnel, 69 & 165 & polymers, 91 \\
\hline air solubility, 1 & impedance, 247 & radiation defects, 139 \\
\hline aluminum calcium foam, 231 & interfacial regions, 155 & roll velocity, 77 \\
\hline atomic-force microscope, 299 & ion-track technology, 139 & shape memory alloys, 165 \\
\hline bound rubber, 31 & laminated object & sheet conjugation, 77 \\
\hline carbon fibers, 19 & manufacturing, 77 & shungite, 31 \\
\hline carbon nanotubes, 117 & lid-driven flow, 343 & silver carbonate, 9 \\
\hline Casson nanofluid, 207 & Lie group transformation, 47 & Sisko non-Newtonian fluid, \\
\hline chemical reaction, 207 & low-angle boundaries, 283 & 247 \\
\hline coating, 91 & lower critical solution & slip velocity, 47 \\
\hline combustion of nanoadditives, & temperature, 91 & solid elastic body, 299 \\
\hline 69 & lubricant film, 9 & solutions, 91 \\
\hline compressive properties, 231 & magnetohydrodynamics, & stability, 299 \\
\hline contact patch, 77 & 343 & stenosis hemodynamics, 247 \\
\hline creation of layered & mechanical properties, 31 & strength, 19 \\
\hline composite, 77 & metallic foam, 231 & stress relieving annealing, \\
\hline crystal plasticity, 283 & micropolar nanofluid, 343 & 231 \\
\hline diffusion time, 1 & microporous media, 183 & stress state type, 165 \\
\hline discrete system of material & microstructure, 231 & stretching cylinder, 99 \\
\hline points, 299 & mixed convection, 343 & stretching sheet, 47 \\
\hline dynamic equilibrium, 1 & mixture rule, 155 & stretching surface, 325 \\
\hline $\begin{array}{l}\text { effective damping properties, } \\
117\end{array}$ & $\begin{array}{l}\text { modulus of elasticity, } 155 \\
\text { morphology, } 231\end{array}$ & $\begin{array}{l}\text { structural transformation, } 165 \\
\text { structure, } 155\end{array}$ \\
\hline elastomer reinforcement, 31 & nanobubble, 1 & subgrains misorientation, \\
\hline electrochemical deposition, & nanocomposites, 19, 31, 155 & 283 \\
\hline 139 & nanofiller efficiency, 155 & suction/injection, 47, 99 \\
\hline epoxy composite, 9 & nanofluid, 47, 99 & summary reaction kinetics, \\
\hline epoxy polymer, 155 & nanoparticles, 247 & 77 \\
\hline $\begin{array}{l}\text { fiber-reinforced polymer } \\
\text { composites, } 117\end{array}$ & $\begin{array}{l}\text { nanostructures, } 139 \\
\text { nanotubes, } 139\end{array}$ & $\begin{array}{l}\text { supersonic flow, } 69 \\
\text { surface tension, } 1\end{array}$ \\
\hline filled rubber, 31 & nonlinear radiative heat flux, & Taylor series expansion and \\
\hline finite element method, 247 & 207 & least squares-based lattice \\
\hline fractal dimensionality, 155 & nonlinear stretching sheet, & Boltzmann method, 183 \\
\hline fragmentation, 283 & 207 & temperature field, 77 \\
\hline friction coefficient, 9 & nonlocal many-particle & template synthesis, 139 \\
\hline FTCS numerical method, 247 & interaction, 299 & thermal radiation, 325 \\
\hline fuzzy fibers, 117 & nonuniform grids, 183 & thermodynamics, 299 \\
\hline generalized self-consistent & numerical analysis, 19 & thermophoresis, 325 \\
\hline method, 117 & numerical solution, 207 & trapezoidal enclosure, 343 \\
\hline growth mechanisms, 139 & ohmic-viscous dissipation, 99 & viscous dissipation, 325 \\
\hline heat and mass transfer, 247 & organoclay, 155 & wear intensity, 9 \\
\hline heat generation/absorption, & pharmacodynamic & wear mechanism, 9 \\
\hline 99,325 & simulation, 247 & $\mathrm{ZnO}$ nanowires, 117 \\
\hline
\end{tabular}

